\title{
STABILITY IN THE HIGH-DIMENSIONAL COHOMOLOGY OF CONGRUENCE SUBGROUPS
}

\author{
JEREMY MILLER, ROHIT NAGPAL, AND PETER PATZT
}

\begin{abstract}
We prove a representation stability result for the codimension-one cohomology of the level three congruence subgroup of $\mathbf{S L}_{n}(\mathbb{Z})$. This is a special case of a question of Church-FarbPutman which we make more precise. Our methods involve proving finiteness properties of the Steinberg module for the group $\mathbf{S L}_{n}(K)$ for $K$ a field. This also lets us give a new proof of AshPutman-Sam's homological vanishing theorem for the Steinberg module. We also prove an integral refinement of Church-Putman's homological vanishing theorem for the Steinberg module for the group $\mathbf{S L}_{n}(\mathbb{Z})$.
\end{abstract}

\section{Contents}

1. Introduction 1

2. The apartment and Steinberg monoids 6

3. Koszulness of the Steinberg monoid of a field 10

4. Finiteness properties of resolutions of the Steinberg module 18

5. Codimension one cohomology of the level 3 congruence subgroup 19

6. A stability conjecture 29

7. Homological vanishing for the Steinberg module 31

$\begin{array}{ll}\text { References } & 38\end{array}$

\section{INTRODUCTION}

The purpose of this paper is to explore finiteness properties of Steinberg modules of special linear groups and applications of these finiteness properties to group (co)homology. In particular, for $K$ a field, we prove that the Steinberg modules of $\mathbf{S L}_{n}(K)$ exhibit a derived form of representation stability (see Theorem 1.5). The primary application of this result is Theorem 1.1 concerning the high degree cohomology of congruence subgroups of $\mathbf{S L}_{n}(\mathbb{Z})$. We also apply our results to reprove a result of Ash-Putman-Sam [APS18] and strengthen a result of Church-Putman [CP17].

1.1. Cohomology of congruence subgroups. The (co)homology of arithmetic groups is a rich subject that has had many applications in number theory and algebraic K-theory. In this paper,

Date: May 14, 2020.

2010 Mathematics Subject Classification. 11F75, 55N25, 20H05, 19B14, 16S37.

Key words and phrases. Congruence subgroups, Koszul algebras, representation stability, Borel-Serre duality.

Jeremy Miller was supported in part by NSF grant DMS-1709726. 
we focus on congruence subgroups of $\mathbf{S L}_{n}(\mathbb{Z})$. Let $\Gamma_{n}(p)$ denote the kernel of the reduction $\bmod p$ map

$$
\mathbf{S L}_{n}(\mathbb{Z}) \rightarrow \mathbf{S L}_{n}\left(\mathbb{F}_{p}\right)
$$

For $i$ small compared to $n$, which we shall refer to as the low-dimensional case, the rational cohomology groups $\mathrm{H}^{i}\left(\Gamma_{n}(p) ; \mathbb{Q}\right)$ are completely known by the work of Borel [Bor74]. From this calculation, one sees that congruence subgroups exhibit rational homological stability.

Our work focuses on integral (co)homology, so all homology and cohomology groups will have integer coefficients unless otherwise specified. The torsion in the integral (co)homology of $\Gamma_{n}(p)$ is quite complicated even in the low-dimensional case and there are hardly any explicit calculations. For $n$ sufficiently large, the groups $\mathrm{H}_{1}\left(\Gamma_{n}(p)\right)$ and $\mathrm{H}_{2}\left(\Gamma_{n}(p)\right)$ were respectively computed by LeeSzczarba [LS76b] and F. Calegari [Cal15]. Even $\mathrm{H}_{3}\left(\Gamma_{n}(p)\right)$ is currently unknown for large $n$. There are however homological and representational stability results for the torsion in the low-dimensional homology of congruence subgroups [Cha84, Put15, CEFN14, CE17, PS17, MPW19, CMNR18, GL19].

In contrast to this complete calculation of the stable rational cohomology and stability results for the torsion, very little is known about the cohomology outside of the stable range, even rationally. Let $p$ be an odd prime. The virtual cohomological dimension, denoted vcd, of $\Gamma_{n}(p)$ is known to be $\left(\begin{array}{l}n \\ 2\end{array}\right)$. Since the cohomology groups $\mathrm{H}^{i}\left(\Gamma_{n}(p)\right)$ vanish if $i>\operatorname{vcd}$, we shall refer to $\mathrm{H}^{\mathrm{vcd}-i}\left(\Gamma_{n}(p)\right)$ as the codimension $i$ cohomology. When $i$ is small compared to $n$, we informally call this the high-dimensional case. This case is even more mysterious than the low-dimensional case and the only calculations known (rational or integral), for a general $n$, exist only in codimension-zero and levels 2, 3, and 5 (Lee-Szczarba [LS76b] and [MPP]). In particular, Lee-Szczarba [LS76b]) made the following computation:

$$
\mathrm{H}^{\mathrm{vcd}}\left(\Gamma_{n}(p)\right) \cong \mathrm{St}_{n}\left(\mathbb{F}_{p}\right) \text { for } n \geq 3 \text { and } p=3 .
$$

Here $\mathrm{St}_{n}(R)$ denotes the Steinberg module of $\mathbf{S L}_{n}(R)$. In a series of papers, Ash-GunnellsMcConnell have calculated the codimension-one cohomology of certain finite index subgroups of $\mathbf{S L}_{4}(\mathbb{Z})$; see [AGM02, AGM08, AGM10].

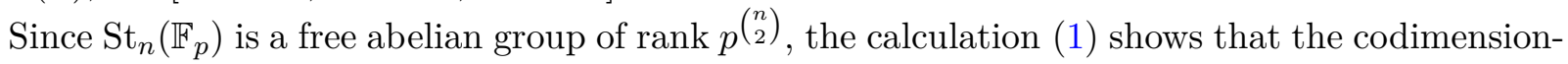
zero cohomology does not stabilize in the classical sense. However, for $n \geq 3$, it does admit a uniform description independent of $n-$ it is the Steinberg module for each $n$. This is the key feature of representation stability.

There are many different notions of representation stability with the most basic being finite generation degree. Let

$$
G_{0} \hookrightarrow G_{1} \hookrightarrow G_{2} \hookrightarrow \ldots
$$

be a sequence of groups and let

$$
M_{0} \rightarrow M_{1} \rightarrow M_{2} \rightarrow \ldots
$$

be a sequence with $M_{n}$ a $G_{n}$-representation and with $M_{n} \rightarrow M_{n+1}$ a $G_{n}$-equivariant map. We say $\left\{M_{n}\right\}_{n \geq 0}$ has generation degree $\leq d$ if

$$
\operatorname{Ind}_{G_{n}}^{G_{n+1}} M_{n} \rightarrow M_{n+1}
$$

is surjective for $n \geq d$. This is the definition of representation stability that we will use. 
Note that $\mathrm{H}^{*}\left(\Gamma_{n}(p)\right)$ has a natural $\mathbf{G L}_{n}^{ \pm}\left(\mathbb{F}_{p}\right)$ action. Here the superscript \pm means we restrict to matrices with determinant equal to \pm 1 . Using Borel-Serre duality [BS73], one can construct equivariant maps

$$
\mathrm{H}^{\mathrm{vcd}-i}\left(\Gamma_{n}(p)\right) \rightarrow \mathrm{H}^{\mathrm{vcd}-i}\left(\Gamma_{n+1}(p)\right)
$$

and thus one can make sense of the generation degree of the codimension- $i$ cohomology of congruence subgroups. Our main theorem is the following.

Theorem 1.1. The sequence $\left\{\mathrm{H}^{\mathrm{vcd}-1}\left(\Gamma_{n}(3)\right)\right\}_{n \geq 0}$ has generation degree $\leq 4$. In other words, the $\mathbf{G L}_{n}\left(\mathbb{F}_{3}\right)$-equivariant map

$$
\operatorname{Ind}_{\mathbf{G L}_{4}\left(\mathbb{F}_{3}\right)}^{\mathbf{G L}_{n}\left(\mathbb{F}_{3}\right)} \mathrm{H}^{\left(\begin{array}{l}
4 \\
2
\end{array}\right)-1}\left(\Gamma_{4}(3)\right) \rightarrow \mathrm{H}^{\left(\begin{array}{c}
n \\
2
\end{array}\right)-1}\left(\Gamma_{n}(3)\right)
$$

is surjective for $n \geq 4$.

In Proposition 6.2, we also show that $\left\{\mathrm{H}^{\mathrm{vcd}}\left(\Gamma_{n}(p)\right)\right\}_{n \geq 0}$ has generation degree equal to 0 for all $p$, although this follows fairly quickly from known results. Finite generation degree allows one to control how fast the dimensions grow. In particular, Theorem 1.1 has the following corollary.

Corollary 1.2. Let $\mathbf{k}$ be a field. Then for $n \geq 4$, we have that

$\operatorname{dim}_{\mathbf{k}} \mathrm{H}^{\mathrm{vcd}-1}\left(\Gamma_{n}(3) ; \mathbf{k}\right) \leq \frac{3^{\left(\begin{array}{c}n-4 \\ 2\end{array}\right)}\left|\mathbf{G L}_{n}\left(\mathbb{F}_{3}\right)\right|}{\left|\mathbf{G L}_{n-4}\left(\mathbb{F}_{3}\right)\right|\left|\mathbf{G L}_{4}\left(\mathbb{F}_{3}\right)\right|} \operatorname{dim}_{\mathbf{k}} \mathrm{H}^{5}\left(\Gamma_{4}(3) ; \mathbf{k}\right) \leq \frac{3^{\left({ }^{n-4}{ }^{2}\right)}\left|\mathbf{G L}_{n}\left(\mathbb{F}_{3}\right)\right|}{\left|\mathbf{G L}_{n-4}\left(\mathbb{F}_{3}\right)\right|\left|\mathbf{G L}_{4}\left(\mathbb{F}_{3}\right)\right|} 227340$.

Remark 1.3. We note that it follows from the work of Ash in [Ash94] that the maps

$$
\mathrm{H}^{\mathrm{vcd}-i}\left(\Gamma_{n}(p)\right) \rightarrow \mathrm{H}^{\mathrm{vcd}-i}\left(\Gamma_{n+1}(p)\right)
$$

are injective. Since $v c d\left(\Gamma_{2}(p)\right)=1$, we have:

$$
\mathrm{H}^{\mathrm{vcd}-1}\left(\Gamma_{2}(p)\right)=\mathrm{H}^{0}\left(\Gamma_{2}(p)\right) \cong \mathbb{Z}
$$

and so $\mathrm{H}^{\mathrm{vcd}-1}\left(\Gamma_{n}(p)\right)$ contains a subgroup isomorphic to $\mathbb{Z}$ for all $n \geq 2$. By the work of LeeSzczarba [LS76b, Theorem 1.5],

$$
\mathrm{H}^{\mathrm{vcd}-1}\left(\Gamma_{3}(3)\right) \cong \mathbb{Z}^{26} \oplus(\mathbb{Z} / 3)^{8}
$$

and so $\mathrm{H}^{\mathrm{vcd}-1}\left(\Gamma_{n}(3)\right)$ contains a subgroup isomorphic to $\mathbb{Z}^{26} \oplus(\mathbb{Z} / 3)^{8}$ for all $n \geq 3$. In particular, Theorem 1.1 concerns nonzero groups.

Theorem 1.1 can be viewed as a special case of a conjecture of Church-Farb-Putman [CFP14]. We pose a refined version of this conjecture in $\S 6$.

Although Theorem 1.1 appears to be the first representation stability result concerning cohomology groups near the virtual cohomological dimension, we expect similar patterns to exist more generally. For example, we would be interested in knowing if representation stability results hold in other contexts such as congruence subgroups of mapping class groups or pure braid groups. 
1.2. Finiteness properties of the Steinberg module. As mentioned earlier, our results on the cohomology of congruence subgroups are powered by stability results for Steinberg modules. To state these results, we need to recall a certain categorical framework. Let $\prod_{n \geq 0} \operatorname{Mod}_{\mathbb{Z}\left[\mathbf{G L}_{n}(R)\right]}$ denote the product of the categories of $\mathbb{Z}\left[\mathbf{G L}_{n}(R)\right]$-modules. There is a natural symmetric monoidal structure on this category often called the convolution product given by the formula

$$
(M \otimes N)_{n}:=\bigoplus_{i+j=n} \operatorname{Ind}_{\mathbf{G L}_{i}(R) \times \mathbf{G L}_{j}(R)}^{\mathbf{G} \mathbf{L}_{n}(R)} M_{i} \otimes_{\mathbb{Z}} N_{j}
$$

where $M=\left\{M_{n}\right\}_{n \geq 0}$ and $N=\left\{N_{n}\right\}_{n \geq 0}$ are two sequences of representations in $\prod_{n \geq 0} \operatorname{Mod}_{\mathbb{Z}\left[\mathbf{G} \mathbf{L}_{n}(R)\right]}$. This product is the same as the Day convolution product induced by the direct sum monoidal structure on the groupoid of finite-rank free $R$-modules. Let St denote the sequence $\left\{\operatorname{St}_{n}(R)\right\}_{n \geq 0}$. Since $\mathbf{G L}_{n}(R)$ acts on $\operatorname{St}_{n}(R)$, we can regard St as an object in the category $\prod_{n \geq 0} \operatorname{Mod}_{\mathbb{Z}\left[\mathbf{G} \mathbf{L}_{n}(R)\right]}$. In fact, St is a monoid object in this category with respect to this monoidal structure and for this reason we call St the Steinberg monoid. One can extend the classical notion of a Koszul algebra from commutative algebra to the general setting of monoidal categories. We show that St is Koszul in this sense. See $§ 3.1$ for further discussion of Koszulness.

Theorem 1.4. Let $K$ be a field. Then $\mathrm{St}=\left\{\mathrm{St}_{n}(K)\right\}_{n \geq 0}$ is Koszul in the monoidal category $\left(\prod_{n \geq 0} \operatorname{Mod}_{\mathbb{Z}\left[\mathbf{G L}_{n}(K)\right]}, \otimes\right)$.

Our proof involves showing that St is a "Poincaré-Birkhoff-Witt"-monoid which allows us to follow an argument due to Priddy (see [Pri70]) to prove our Koszulness result.

Let $\mathbf{A}=\bigwedge\left(\operatorname{triv}_{1}\right)$ be the exterior algebra on $\operatorname{triv}_{1}=\left\{E_{n}\right\}_{n \geq 0}$ where

$$
E_{n}:= \begin{cases}0 & \text { if } n \neq 1 \\ \mathbb{Z} & \text { if } \left.n=1 \text { (with the trivial action of } \mathbf{G L}_{1}(K)\right) .\end{cases}
$$

By exterior algebra, we mean the free skew commutative monoid with respect to this monoidial structure. We call this exterior algebra the apartment monoid and construct a surjective map $\mathbf{A} \rightarrow$ St of monoids (surjectivity of this map reflects the fact that $\operatorname{St}_{n}(K)$ is generated by apartment classes; see Remark 2.7 for more on this). Koszulness of exterior algebras is well-known (see the paragraphs after Theorem 3.1), and so the theorem above tells us that $\mathbf{A} \rightarrow$ St is a map of Koszul monoids. We prove a general technical result (Proposition 4.6) on maps of Koszul monoids which we expect to be useful in many other situations. This result applied to the map $\mathbf{A} \rightarrow$ St proves the following finiteness theorem which is the main technical result of our paper.

Theorem 1.5. For a field $K$, then $\operatorname{Tor}_{i}^{\mathbf{A}}(\mathrm{St}, \mathbb{Z})$ (regarded as a graded abelian group) is supported in degrees $\leq 2 i$.

Let $\mathbf{A}$ be the apartment monoid defined above for $K=\mathbb{F}_{3}$. The sequence $\left\{\mathrm{H}_{i}\left(\Gamma_{n}(3) ; \mathrm{St}_{n}(\mathbb{Z})\right)\right\}_{n \geq 0}$ forms an A-module which we call $\mathrm{H}_{i}(\Gamma(3) ; \mathrm{St}(\mathbb{Z}))$. Using Borel-Serre duality, Theorem 1.1 can be rephrased as saying that

$$
\operatorname{Tor}_{0}^{\mathbf{A}}\left(\mathrm{H}_{1}(\Gamma(3) ; \mathrm{St}(\mathbb{Z})), \mathbb{Z}\right)
$$

is supported in degree $\leq 4$ (see Proposition 5.14). The monoid A plays a similar role in this paper as FI plays in Church-Ellenberg-Farb [CEF15] and VIC and SI play in Putman-Sam [PS17]; 
the category of A-modules is the category governing representation stability in this context. To prove Theorem 1.1, we will only need Theorem 1.5 (and hence Theorem 1.4) for $K=\mathbb{F}_{3}$. However, Koszulness of the Steinberg monoid in the general case lets us provide a new proof of the following theorem due to Ash-Putman-Sam.

Theorem 1.6 ([APS18]). $\mathrm{H}_{i}\left(\mathbf{G L}_{n}(K) ; \mathrm{St}_{n}(K)\right)=0$ for all $n \geq 2 i+2$ where $K$ is a field and $\mathrm{St}_{n}(K)$ is the Steinberg module for the group $\mathbf{S L}_{n}(K)$.

Remark 1.7. We have recently been informed that our Koszulness result for the Steinberg monoid is the input needed for the $E_{k}$-algebra stability machine of Galatius-Kupers-Randal-Williams [GKRW18] to apply to homology with coefficients in Steinberg modules. In particular, using our result they were able to improve the work of Ash-Putman-Sam and prove slope $2 / 3$ vanishing range for $\mathrm{H}_{*}\left(\mathbf{G L}_{n}(K) ; \mathrm{St}_{n}(K)\right)$ for $K$ a field.

Our new proof of Theorem 1.6 follows from a more general result on Koszul monoids.

Theorem 1.8. Let $A$ be a (skew) commutative Koszul monoid in $\prod_{n \geq 0} \operatorname{Mod}_{\mathbb{Z}\left[\mathbf{G} \mathbf{L}_{n}(R)\right]}$ where $R$ is a PID. Assume that the following holds:

(a) $\mathrm{H}_{0}\left(\mathbf{G L}_{2}(R) ; A_{2}\right)=0$.

(b) The product map $\mathrm{H}_{0}\left(\mathbf{G L}_{1}(R) ; A_{1}\right) \otimes \mathrm{H}_{1}\left(\mathbf{G L}_{2}(R) ; A_{2}\right) \rightarrow \mathrm{H}_{1}\left(\mathbf{G L}_{3}(R) ; A_{3}\right)$ is surjective.

Then we have the following:

(a') The product map $\mathrm{H}_{0}\left(\mathbf{G L}_{1}(R) ; A_{1}\right) \otimes \mathrm{H}_{i}\left(\mathbf{G L}_{n-1}(R) ; A_{n-1}\right) \rightarrow \mathrm{H}_{i}\left(\mathbf{G L}_{n}(R) ; A_{n}\right)$ is surjective for $n \geq 2 i+1$.

(b') $\mathrm{H}_{i}\left(\mathbf{G L}_{n}(R) ; A_{n}\right)=0$ in degrees $n \geq 2 i+2$.

Theorem 1.8 can be generalized by replacing the groupoid $\left\{\mathbf{G L}_{n}(R)\right\}_{n \geq 0}$ with other braided monoidal groupoids such as the groupoids formed by braid groups or symmetric groups. In this generality, one can see that Hypotheses (a) and (b) are necessary by considering symmetric groups and the sign representation. See Remark 7.3.

It is not known whether St is Koszul in $\prod_{n \geq 0} \operatorname{Mod}_{\mathbb{Z}\left[\mathbf{G L}_{n}(\mathbb{Z})\right]}$. In particular, the analogue of Theorem 1.6 for $\mathbf{G L}_{n}(\mathbb{Z})$ is not known. However, Church-Putman proved the following homological vanishing theorem for $i=1$.

Theorem 1.9 (Church-Putman, Theorem A [CP17]). For $\mathbf{k}$ a field of characteristic zero, we have:

$$
\begin{gathered}
\mathrm{H}_{1}\left(\mathbf{G L}_{n}(\mathbb{Z}) ; \mathrm{St}_{n}(\mathbb{Z}) \otimes_{\mathbb{Z}} \mathbf{k}\right)=0 \text { for } n \geq 0, \\
\mathrm{H}_{1}\left(\mathbf{S L}_{n}(\mathbb{Z}) ; \mathrm{St}_{n}(\mathbb{Z}) \otimes_{\mathbb{Z}} \mathbf{k}\right)=0 \text { for } n \geq 3,
\end{gathered}
$$

We use our main result, Theorem 1.1, to prove the following integral refinement.

Theorem 1.10. Let $\mathbf{k}$ be an arbitrary commutative ring. For $n \geq 6$, we have that:

$$
\begin{aligned}
\mathrm{H}_{1}\left(\mathbf{G L}_{n}(\mathbb{Z}) ; \mathrm{St}_{n}(\mathbb{Z}) \otimes_{\mathbb{Z}} \mathbf{k}\right) & =0, \\
\mathrm{H}_{1}\left(\mathbf{S L}_{n}(\mathbb{Z}) ; \mathrm{St}_{n}(\mathbb{Z}) \otimes_{\mathbb{Z}} \mathbf{k}\right) & =0 .
\end{aligned}
$$

Remark 1.11. Although $\mathbf{G L}_{n}(\mathbb{Z})$ is only a duality group with $\mathbb{Q}$ coefficients and, for $n$ even, the Steinberg module is not the dualizing module (see Putman-Studenmund [PS] for a description of 
the dualizing module), the groups $\mathrm{H}_{i}\left(\mathbf{G L}_{n}(\mathbb{Z}) ; \mathrm{St}_{n}(\mathbb{Z})\right)$ are still of interest. For $R$ a ring of integers in a number field, there is a spectral sequence due to Quillen [Qui73] with

$$
E_{a b}^{1}=\mathrm{H}_{b}\left(\mathbf{G L}_{a}(R) ; \mathrm{St}_{a}(R)\right) \Longrightarrow \mathrm{H}_{a+b}\left(\Omega^{\infty-1}(K(R))\right) .
$$

Here $\Omega^{\infty-1}(K(R))$ denotes the one-fold delooping of the infinite loop space associated to the algebraic K-theory spectrum of $R$. Many of the calculations of algebraic K-groups using this spectral sequence have relied on homological vanishing results to simplify the $E^{1}$-page (see e.g. [LS76a, LS78, DSGG ${ }^{+}$19, SEVKM]). We hope this new vanishing result will also be useful for computations.

Outline of the paper. In $\S 2$, we discuss algebraic preliminaries and define the Steinberg monoid and the apartment monoid. In $\S 3$, we prove our Koszulness result for the Steinberg monoid of a field (Theorem 1.4). In §4, we use Koszulness of the Steinberg monoid to establish finiteness properties of the Steinberg monoid viewed as a module over the apartment monoid. In $\S 5$, we use these finiteness properties to prove our main representation stability result for the codimension-one cohomology of level 3 congruence subgroups (Theorem 1.1). We pose a refined version of a conjecture due to Church-Farb-Putman in $\S 6$ that generalizes Theorem 1.1 to all primes, all codimensions, and also addresses a more robust notion of representation stability. Finally, in $\S 7$, we give a new proof of the homological vanishing theorem of Ash-Putman-Sam (Theorem 1.6), and an integral refinement of Church-Putman's homological vanishing theorem (Theorem 1.10).

Acknowledgments. We thank Avner Ash, Martin Bendersky, Thomas Church, Benson Farb, Paul Gunnells, Richard Hepworth, Alexander Kupers, Mark McConnell, Andrew Putman, Steven Sam, David Sprehn, and Jennifer Wilson for helpful conversations.

\section{The apartment And Steinberg monoids}

In this section, we define the apartment monoid and the Steinberg monoid. We begin by discussing the category of VB-modules and its symmetric monoidal product.

2.1. VB-modules. Fix a commutative base ring $R$ and a commutative coefficient ring $\mathbf{k}$. Let VB (or $\mathrm{VB}_{R}$ when we need to be more precise) be the groupoid whose objects are finite-rank free $R$-modules and whose morphisms are $R$-linear bijections. There is a natural symmetric monoidal structure $\oplus$ on VB given by direct sum. A VB-module is a covariant functor VB $\rightarrow \operatorname{Mod}_{\mathbf{k}}$. The symmetric monoidal structure $\oplus$ on VB induces a symmetric monoidal structure $\otimes$ on ModVB $_{\text {Viven }}$ by:

$$
(M \otimes N)(X)=\bigoplus_{X_{1} \oplus X_{2}=X} M\left(X_{1}\right) \otimes_{\mathbf{k}} N\left(X_{2}\right) .
$$

To be more precise, in the expression above $X_{1}, X_{2}$ vary over free submodules of $X$ such that every element of $X$ can be written uniquely as a sum of a vector in $X_{1}$ and a vector in $X_{2}$. For $R$ a field, this reduces to the condition that $X_{1}+X_{2}=X$ and $X_{1} \cap X_{2}=0$. See Remark 2.1 for further discussion. We reserve the symbol $\otimes$ without subscript for this product. Note that if $X_{1}$ and $X_{2}$ are rank $n$ free $R$-modules then there is a VB-isomorphism $X_{1} \rightarrow X_{2}$. We define $M(n)$ to be $M\left(R^{n}\right)$. We will often denote $\mathbf{G L}\left(R^{n}\right)=\mathbf{G L}_{n}(R)$ by $\mathbf{G L}_{n}$ when the ring is understood. There is an action of $\mathbf{G L}_{n}$ on $M(n)$ and a natural isomorphism: 


$$
(M \otimes N)(n) \cong \bigoplus_{i+j=n} \operatorname{Ind}_{\mathbf{G} \mathbf{L}_{i} \times \mathbf{G} \mathbf{L}_{j}}^{\mathbf{G} \mathbf{L}_{n}} M(i) \otimes_{\mathbf{k}} N(j) .
$$

The support of a VB-module $M$ is the set of non-negative integers such that $M(n)$ is not zero. We say that $M$ is supported on a set if the support is contained in that set. If the support of $M$ is non-empty, we define the degree of $M$ to be the supremum of the support and otherwise define it to be -1 . We denote the degree of $M$ by $\operatorname{deg} M$.

The category Mod $\mathrm{VB}$ of VB-modules is easily seen to be equivalent to the product category $\prod_{n \geq 0} \operatorname{Mod}_{\mathbf{k}\left[\mathbf{G L}\left(R^{n}\right)\right]}$, and so $\operatorname{Mod}_{\mathrm{VB}}$ is a Grothendieck category. In particular, it is complete and cocomplete.

Remark 2.1. We note that the monoidal structure $\otimes$ on $\operatorname{Mod}_{\mathrm{VB}}$ is given by the composite:

$$
\operatorname{Mod}_{\mathrm{VB}} \times \operatorname{Mod}_{\mathrm{VB}} \stackrel{\otimes}{\rightarrow} \operatorname{Mod}_{\mathrm{VB} \times \mathrm{VB}} \stackrel{\operatorname{Lan}_{\oplus}}{\longrightarrow} \operatorname{Mod}_{\mathrm{VB}}
$$

where $\otimes i$ is the external tensor product and $\operatorname{Lan}_{\oplus}$ is the left Kan extension along $\oplus: \mathrm{VB} \times \mathrm{VB} \rightarrow \mathrm{VB}$. In particular, $\otimes$ is the tensor product $\otimes_{[\mathrm{VB}, \oplus]}$ defined in $[\mathrm{Dja}]$. This remarks allows us to quote results from $[\mathrm{Dja}]$.

2.2. The apartment monoid. Let $A$ be a monoid object in the category of VB-modules. An $A$ module $M$ is a VB-module together with an action $A \otimes M \rightarrow M$ satisfying the usual associativity and unitality conditions. If $M$ is a right $A$-module and $N$ is a left $A$-module, we define $M \otimes_{A} N$ as the coequalizer of the two natural maps:

$$
M \otimes A \otimes N \rightrightarrows M \otimes N .
$$

Let $\operatorname{Tor}_{*}^{A}(M, N)$ denote the associated left-derived functors. We say that $A$ is skew commutative if $a \wedge b=(-1)^{n m}(b \wedge a)$ whenever $a \in A\left(R^{n}\right), b \in A\left(R^{m}\right)$, where $\wedge: A \otimes A \rightarrow A$ denote the multiplication map. We regard $\mathbf{k}$ as the monoid in $\operatorname{Mod}_{\mathrm{VB}}$ concentrated in degree 0 . All the monoids that we consider in this paper admit a natural surjective map of monoids $A \rightarrow \mathbf{k}$ whose kernel $A_{+}$is supported in degrees $>0$ (see $\S 3.1$ for more on this). In particular, $\mathbf{k}$ will have the structure of an $A$-module and so we can make sense of $\operatorname{Tor}_{*}^{A}(\mathbf{k},-)$.

Let $\Lambda$ denote the left adjoint of the forgetful functor from the category of skew commutative VB-monoids to the category of VB-modules. Let triv 1 be the VB-module such that $\operatorname{triv}_{1}(n)=0$ for $n \neq 1$, and $\operatorname{triv}_{1}(1)$ is the rank 1 trivial representation of $\mathbf{G L}_{1}$. We call the skew commutative VB-monoid $\bigwedge\left(\operatorname{triv}_{1}\right)$ the apartment monoid, and we denote it by A. See Remark 2.7 for a discussion of the name apartment monoid and the connection with apartments in the Tits building. Unraveling the definition, we obtain the following result:

Proposition 2.2. Let $X$ be a free $R$-module of rank $n$. Then $\mathbf{A}(X)$ is a k-module generated by elements of the form $\left[v_{1}, \ldots, v_{n}\right]$, one for each ordered basis $v_{1}, \ldots, v_{n}$ of $X$, subject to the following relations:

(a) $\left[v_{1}, \ldots, v_{n}\right]=\operatorname{sgn}(\sigma)\left[v_{\sigma(1)}, \ldots, v_{\sigma(n)}\right]$ for $\sigma$ a permutation of $\{1,2, \ldots, n\}$.

(b) $\left[r v_{1}, v_{2}, \ldots, v_{n}\right]=\left[v_{1}, \ldots, v_{n}\right]$ for $r \in R^{\times}$. 
Moreover, the multiplication map

$$
\mathbf{A}\left(X_{1}\right) \otimes_{\mathbf{k}} \mathbf{A}\left(X_{2}\right) \rightarrow \mathbf{A}(X)
$$

is given on generators by

$$
\left[u_{1}, \ldots, u_{k}\right]\left[v_{1}, \ldots, v_{n-k}\right]=\left[u_{1}, \ldots, u_{k}, v_{1}, \ldots, v_{n-k}\right] .
$$

In addition to exterior algebras, we will also need tensor algebras and symmetric algebras. The free (commutative) monoid functor is defined to be the left adjoint of the forgetful functor from the category of (commutative) monoid objects in $\operatorname{Mod}_{\mathrm{VB}}$ to $\operatorname{Mod}_{\mathrm{VB}}$. The tensor algebra $\mathrm{T}(M)$ on a VB-module $M$ is the free monoid on $M$. Similarly the symmetric algebra $\operatorname{Sym} M$ is the free commutative monoid on $M$.

Remark 2.3. Monoid objects in a related representation theoretic monoidal category have been studied extensively by Sam-Snowden and others under the name twisted commutative algebras (tcas); see [SS15] for example. It is easy to spot the influence of the theory of tcas in this paper.

2.3. The Steinberg monoid. Let $K$ be a field and $X$ an $n$-dimensional $K$-vector space. The Tits building for $X$ is denoted $\mathcal{T}_{n}(X)$ and is the geometric realization of the poset of nonempty proper subspaces of $X$ ordered by inclusion. Given an integral domain $R$ and a free $R$-module $X$ of rank $n>0$, the Steinberg module with coefficients in $\mathbf{k}$ is defined to be

$$
\operatorname{St}(X):=\widetilde{\mathrm{H}}_{n-2}\left(\mathcal{T}\left(X \otimes_{R} \operatorname{Frac}(R)\right) ; \mathbf{k}\right)
$$

where $\operatorname{Frac}(R)$ denotes the field of fraction. We define $\mathbf{S t}(X)$ to be $\mathbf{k}$ if the rank of $X$ is zero and use the usual convention that the reduced homology of the empty set is the coefficient ring. Note that here we are denoting the Steinberg module by a bold symbol which is in contrast to our notation in the introduction. We do this to take into account that now the coefficients are in a general ring k. We have chosen to introduce generalized coefficients to be able to talk about dimensions and characteristic zero settings.

Since $\mathbf{G L}\left(X \otimes_{R} \operatorname{Frac}(R)\right) \cong \mathbf{G L}_{n}(\operatorname{Frac}(R))$ acts on $\mathcal{T}\left(X \otimes_{R} \operatorname{Frac}(R)\right), \mathbf{S t}(X)$ is a representation of $\mathbf{G L}\left(X \otimes_{R} \operatorname{Frac}(R)\right)$ and hence also a representation of $\mathbf{G L}(X) \cong \mathbf{G L}_{n}(R)$. For $X=R^{n}$, we denote $\mathbf{S t}(X)$ by $\mathbf{S t}_{n}(R)$. We now recall some presentations of Steinberg modules.

Theorem 2.4 (Lee-Szczarba [LS76b, §3]). Let $K$ be a field and $X$ an $n$-dimensional $K$-vector space. As a k-module, $\mathbf{S t}(X)$ is generated by elements of the form $\left[v_{1}, \ldots, v_{n}\right]$, one for each ordered basis $v_{1}, \ldots, v_{n}$ of $X$, subject to the following relations:

(a) $\left[v_{1}, \ldots, v_{n}\right]=\operatorname{sgn}(\sigma)\left[v_{\sigma(1)}, \ldots, v_{\sigma(n)}\right]$ for $\sigma$ a permutation.

(b) $\left[r v_{1}, v_{2}, \ldots, v_{n}\right]=\left[v_{1}, \ldots, v_{n}\right]$ for $r \in K^{\times}$.

(c) $\sum_{i}(-1)^{i}\left[v_{0}, v_{1}, v_{2}, \ldots, \hat{v}_{i}, \ldots, v_{n}\right]=0$ where $v_{0}, \ldots, v_{n}$ are nonzero vectors and terms of the form $\left[v_{0}, v_{1}, v_{2}, \ldots, \hat{v}_{i}, \ldots, v_{n}\right]$ with $v_{0}, v_{1}, v_{2}, \ldots, \hat{v}_{i}, \ldots, v_{n}$ not a basis are omitted from the sum.

The $\mathbf{G L}(V)$ action is given by the formula $g\left[v_{1}, \ldots, v_{n}\right]=\left[g v_{1}, \ldots, g v_{n}\right]$.

Note that relation $(c)$ implies the first two relations. 
Theorem 2.5 (Bykovskii [Byk03]). Let $X$ be a free $\mathbb{Z}$-module of rank $n$. As a $\mathbf{k}$-module, $\mathbf{S t}(X)$ is generated by elements of the form $\left[v_{1}, \ldots, v_{n}\right]$, one for each ordered basis $v_{1}, \ldots, v_{n}$ of $\mathbb{Z}^{n}$, subject to the following relations:

(a) $\left[v_{1}, \ldots, v_{n}\right]=\operatorname{sgn}(\sigma)\left[v_{\sigma(1)}, \ldots, v_{\sigma(n)}\right]$ for $\sigma$ a permutation.

(b) $\left[-v_{1}, v_{2}, \ldots, v_{n}\right]=\left[v_{1}, \ldots, v_{n}\right]$.

(c) $\left[v_{1}, v_{2}, \ldots, v_{n}\right]-\left[v_{0}, v_{2}, \ldots, v_{n}\right]+\left[v_{0}, v_{1}, \ldots, v_{n}\right]=0$ where $v_{0}=v_{1}+v_{2}$.

The $\mathbf{G L}(X)$ action is given by the formula $g\left[v_{1}, \ldots, v_{n}\right]=\left[g v_{1}, \ldots, g v_{n}\right]$.

The Steinberg modules assemble to form a VB-module. We now define a monoid structure on St when $R$ is a field or the integers. We call this the Steinberg monoid.

Proposition 2.6. Let $R$ be $\mathbb{Z}$ or a field and let $X$ and $Y$ be free $R$-modules of rank $n$ and $m$ respectively. The map $\mathbf{S t}(X) \otimes_{\mathbf{k}} \mathbf{S t}(Y) \rightarrow \mathbf{S t}(X \oplus Y)$ given by

$$
\left[v_{1}, \ldots, v_{n}\right] \otimes\left[u_{1}, \ldots, u_{m}\right] \mapsto\left[v_{1}, \ldots, v_{n}, u_{1}, \ldots, u_{m}\right]
$$

is well-defined and gives $\mathbf{S t}$ the structure of a monoid object in $\left(\operatorname{Mod}_{\mathrm{VB}}, \otimes\right)$.

Proof. The only thing that is not trivial is that the function is well-defined. This follows from the above presentations.

A monoid structure on St actually exists for all integral domains but we will not need this. It follows Theorem 2.4 and Theorem 2.5 that for $R$ a field or the integers, there is a natural surjective map $\mathbf{A} \rightarrow \mathbf{S t}$ which is a map of monoids (in fact such a surjection exists for $R$ Euclidean by the work of Ash-Rudolph [AR79]). This gives St the structure of an A-module. In fact, Theorem 2.5 (or Theorem 2.8 in the field case) immediately implies that $\mathbf{S t}$ is a quotient of $\mathbf{A}$ by a two-sided ideal generated in degree 2 (the additional relation (c) only depends on $v_{0}, v_{1}, v_{2}$ which span a rank 2 summand). From this perspective the assertion of the proposition above is immediate as well.

Remark 2.7. For every integral domain $R$, there is a natural map $\mathbf{A} \rightarrow \mathbf{S t}$ which is not always surjective. The image of a generator $\left[v_{1}, \ldots, v_{n}\right]$ in $\widetilde{\mathrm{H}}_{n-2}\left(\mathbf{S t}_{n}(R)\right)$ is known as an apartment class and is the fundamental class of a sphere in $\mathcal{T}\left(\operatorname{Frac}(R)^{n}\right)$ known as an apartment. The apartment associated to $\left[v_{1}, \ldots, v_{n}\right]$ is the full subcomplex of $\mathcal{T}\left(\operatorname{Frac}(R)^{n}\right)$ with vertices given by the span of nonempty proper subsets of $\left\{v_{1}, \ldots, v_{n}\right\}$. When $R$ is the ring of integers in $\operatorname{Frac}(R)$, these classes are known as integral apartment classes. It is an interesting question to classify when the Steinberg module is generated by integral apartment classes. See [AR79, CFP19, MPWY] for more on this question.

The following theorem seems to be known to experts but we could not find a reference for it in the literature so we will sketch a proof.

Theorem 2.8. Let $K$ be a field and $X$ an $n$-dimensional $K$-vector space. As a $\mathbf{k}$-module, $\mathbf{S t}(X)$ is generated by elements of the form $\left[v_{1}, \ldots, v_{n}\right]$, one for each basis $v_{1}, \ldots, v_{n}$ of $X$, subject to the following relations:

(a) $\left[v_{1}, \ldots, v_{n}\right]=\operatorname{sgn}(\sigma)\left[v_{\sigma(1)}, \ldots, v_{\sigma(n)}\right]$ for $\sigma$ a permutation.

(b) $\left[r v_{1}, v_{2}, \ldots, v_{n}\right]=\left[v_{1}, \ldots, v_{n}\right]$ for $r \in K^{\times}$. 
(c) $\left[v_{1}, v_{2}, \ldots, v_{n}\right]-\left[v_{0}, v_{2}, \ldots, v_{n}\right]+\left[v_{0}, v_{1}, \ldots, v_{n}\right]=0$ where $v_{0}=v_{1}+v_{2}$.

The $\mathbf{G L}(X)$ action is given by the formula $g\left[v_{1}, \ldots, v_{n}\right]=\left[g v_{1}, \ldots, g v_{n}\right]$.

Proof. Call $S(X)$ the $\mathbf{k}[\mathbf{G L}(X)]$-module with the above presentation. By Theorem 2.4, sending a generator to the generator with the same name gives a well-defined surjective map $f: S(X) \rightarrow$ $\operatorname{St}(X)$. Pick an isomorphism $X \cong K^{n}$. To see that $f$ is injective, note that we can use relations (a), (b), and (c) to show that $S\left(K^{n}\right)$ is generated as a k-module by symbols $\left[v_{1}, v_{2}, \ldots, v_{n}\right]$ where the vectors assemble to form a unit upper triangular matrix with respect to the standard basis of $K^{n}$. The Solomon-Tits theorem (see [Bro89, Theorem IV.5.2]) implies that $\mathbf{S t}_{n}(K)$ has a basis given by unit upper triangular matrices. Thus, $f$ is also injective.

\section{Koszulness of the Steinberg monoid of A Field}

In this section, we prove that the Steinberg monoid of a field is Koszul. We follow an argument of Priddy [Pri70].

3.1. Preliminaries on Koszulness. In a non-negatively graded monoidal category with unit object $\mathbf{k}$ (which we assume is supported in degree 0), we say that a monoid $A$ is an augmented monoid if there is a surjection $A \rightarrow \mathbf{k}$ of monoids (called the augmentation map) whose kernel $A_{+}$ (called the augmentation ideal) is supported in degrees $>0$. Note that if such an augmentation exists, it will be unique. A map of augmented monoids is a map of monoids that preserves the augmentation map. In particular, the augmentation map defined above is a map of augmented monoids. The apartment and Steinberg monoids are naturally augmented monoids in $\left(\operatorname{Mod}_{\mathrm{VB}}, \otimes\right)$. One can define Koszul monoids in the general setting of symmetric monoidal categories but we discuss it only in the setting of VB-modules for concreteness as follows.

Recall that we regard $\mathbf{k}$ as a VB-module supported in degree 0 . In other words, $\mathbf{k}$ is the unit object in $\operatorname{Mod}_{\mathrm{VB}}$, and so is naturally an augmented monoid. Let $A$ be an augmented monoid in $\left(\operatorname{Mod}_{\mathrm{VB}}, \otimes\right)$. The two-sided reduced bar resolution $\mathcal{B}_{*}(A, A) \rightarrow A \rightarrow 0$ of $A$ is given by

$$
A \otimes \mathrm{T}^{*}\left(A_{+}\right) \otimes A \rightarrow A \rightarrow 0
$$

where $\mathrm{T}($.$) denotes the tensor algebra and \mathrm{T}^{*}($.$) denotes its *$ th graded piece (we have borrowed our notation from [Pri70]). If $M$ is a right $A$-module and $N$ is a left $A$-module, then $\operatorname{Tor}_{*}^{A}(M, N)$ is the homology of

$$
\mathcal{B}_{*}(M, A, N):=M \otimes_{A} \mathcal{B}_{*}(A, A) \otimes_{A} N .
$$

The module $\mathcal{B}_{s}(M, A, N)$ is generated by elements of the form $m \otimes a_{1} \otimes \cdots \otimes a_{s} \otimes n$ where $m \in M$, $n \in N$ and $a_{i} \in A_{+}$. Such elements are written as $m \otimes\left[a_{1}\left|a_{2}\right| \cdots \mid a_{s}\right] \otimes n$ for historical reasons. We shall only need the special case when $M=N=\mathbf{k}$. We denote the complex $\mathcal{B}_{*}(\mathbf{k}, A, \mathbf{k})$ calculating $\operatorname{Tor}_{*}^{A}(\mathbf{k}, \mathbf{k})$ simply by $\overline{\mathcal{B}}_{*}(A)$. In this case, the differential is given by

$$
\partial\left(\left[a_{1}\left|a_{2}\right| \cdots \mid a_{s}\right]\right)=\sum_{j=1}^{s-1}(-1)^{j-1}\left[a_{1}|\cdots| a_{j} a_{j+1}|\cdots| a_{s}\right] .
$$

For every element $\left[a_{1}\left|a_{2}\right| \cdots \mid a_{s}\right] \in \overline{\mathcal{B}}_{*}(A)$, the homological degree is defined to be $s$ and internal degree is defined to be $\sum_{i=1}^{s} \operatorname{deg} a_{i}$. We shall denote the homological degree $s$ and internal degree $n$ piece of $\overline{\mathcal{B}}_{*}(A)$ by $\overline{\mathcal{B}}_{s}^{n}(A)$. We say that $A$ is $\mathbf{K o s z u l}$ if $\operatorname{Tor}_{i}^{A}(\mathbf{k}, \mathbf{k})$ is supported only in internal 
degree $i$ (for each $i \geq 0$ ). Equivalently, $A$ is a Koszul monoid if, for each $n \geq 0$, the homology of $\overline{\mathcal{B}}_{*}^{n}(A)$ is supported in homological degree $n$.

3.2. Koszulness of the apartment and the Steinberg monoids. Recall that the apartment monoid $\mathbf{A}$ is by definition $\bigwedge\left(\operatorname{triv}_{1}\right)$, the exterior algebra on triv 1 . Koszulness of exterior algebras is quite well-known in other contexts and the usual proof carries over to the symmetric monoidal category of VB-modules.

Theorem 3.1 (Koszulness of the apartment monoid). Let $R$ be a PID, and $\mathbf{A}$ be the apartment monoid in $\left(\operatorname{Mod}_{\mathrm{VB}_{R}}, \otimes\right)$. Then $\mathbf{A}$ is Koszul.

We shall not prove Theorem 3.1 but an interested reader can obtain proofs by following the proof of Koszulness of the Steinberg monoid St. Also see the discussion in §3.3. We prove Koszulness of St in the case when $R$ is a field as follows:

- Using the Solomon-Tits theorem, we obtain a basis of the Steinberg module consisting of unit upper triangular matrices.

- We define a well-ordering on the basis of $\overline{\mathcal{B}}_{*}(\mathbf{S t})$ obtained above which makes St a "PoincaréBirkhoff-Witt-like" (PBW-like) monoid (we chose not to make this precise).

- We follow Priddy's argument as in [Pri70] that PBW algebras are Koszul to finish our argument.

Theorem 3.2 (Koszulness of the Steinberg monoid). Suppose $R$ is a field. Then $\operatorname{Tor}_{i}^{\mathbf{S t}}(\mathbf{k}, \mathbf{k})$ is supported in degree $i$.

Fix a field $K$, and assume that $R=K$ throughout the rest of this subsection. Proposition 3.6 below immediately implies the theorem above. We now provide some preliminaries needed for the proposition.

Proposition 3.3. Let $W$ be a d-dimensional $K$-vector space. Let $B=\left(v_{1}, \ldots, v_{d}\right)$ be an ordered basis of $W$, and let $\mathbf{U}_{d}$ be the subgroup of those matrices in $\mathbf{G L}_{d}(K)$ whose matrix with respect to $B$ is unit lower triangular. So if $g \in \mathbf{U}_{d}$ then $g v_{i}=v_{i}+\sum_{j>i} c_{i j} v_{j}$ for some $c_{i j} \in K$. Then $\mathbf{S t}(W)$ is freely generated as a $\mathbf{k}$-module by the apartment classes

$$
\mathrm{PBW}_{W}:=\left\{\left[g v_{1}, \ldots, g v_{d}\right] \mid g \in \mathbf{U}_{d}\right\} .
$$

Proof. This is the Solomon-Tits theorem (see [Bro89, Theorem IV.5.2]).

Remark 3.4. Let $W$ be a d-dimensional subspace of $K^{n}$. Let $B=\left(v_{1}, \ldots, v_{d}\right)$ be an ordered basis of $W$, and let $M$ be a matrix whose $i$-th column is $v_{i}$ written in the standard basis of $K^{n}$. Then $\mathrm{PBW}_{W}$ consists of column vectors in matrices obtained by multiplying $M$ with a $d \times d$ unit lower triangular matrix on the right.

To prove Theorem 3.2, it suffices to show that the homology of $\overline{\mathcal{B}}_{*}^{n}(\mathbf{S t})$ is supported in homological degree $n$. We now describe $\overline{\mathcal{B}}_{*}^{n}(\mathbf{S t})$ in more detail. Since we are only interested in internal degree $n$, we can work with the $K$-vector space $K^{n}$ and its subspaces. In particular, we note that

$$
\overline{\mathcal{B}}_{s}^{n}(\mathbf{S t})=\underset{\bigoplus_{j=1}^{s} W_{j}=K^{n}}{\bigoplus_{1}} \mathbf{S t}\left(W_{1}\right) \otimes_{\mathbf{k}} \cdots \otimes_{\mathbf{k}} \mathbf{S t}\left(W_{s}\right)
$$


where we note that a simple tensor $a_{W_{1}} \otimes \cdots \otimes a_{W_{s}}$ in $\mathbf{S t}\left(W_{1}\right) \otimes_{\mathbf{k}} \cdots \otimes_{\mathbf{k}} \mathbf{S t}\left(W_{s}\right)$ is denoted as $\left[a_{W_{1}}|\cdots| a_{W_{s}}\right]$ as is conventional for the Bar construction. Given a subspace $W$ of $K^{n}$, we describe below a PBW-like basis of $\mathbf{S t}(W)$. Let the dimension of $W$ be $d \leq n$. We now assign to $W$ a canonical subset $S_{W} \subset[n]$ of size $d$, a canonical $K$-basis $B_{W}$ of $W$, and a canonical PBW-like k-basis, denoted $\mathrm{PBW}_{W}$, of $\mathbf{S t}(W)$ :

(a) Let $v_{1}, \ldots, v_{d}$ be a basis of $W$. Put $v_{1}, \ldots, v_{d}$ as column vectors (with respect to the standard basis of $\left.K^{n}\right)$ in an $n \times d$ matrix $M$.

(b) Let $N$ be the column-reduced Echelon form of $M$. Recall that the column-reduced Echelon form (transpose of a row-reduced Echelon form) is unique and does not depend on the choice of $v_{1}, \ldots, v_{d}$ made earlier.

(c) Define $S_{W}$ to be the set of row indices that contain a leading one in $N$, and define $B_{W}$ to be the columns of $N$. Note that there is a natural ordering on $B_{W}$ coming from column indices.

(d) Define $\mathrm{PBW}_{W}$ to be the basis as described in Proposition 3.3 with respect to the ordered basis $B_{W}$.

Note here that we can think of $S_{W}$ as the index of the lexicographically least nonzero Plückercoordinate of $W$. As an example, suppose $n=4$ and let $W$ be the subspace of $K^{4}$ of dimension $d=3$ generated by the columns of the following matrix $M$ :

$$
M=\left[\begin{array}{lll}
1 & 0 & 0 \\
2 & 0 & 0 \\
0 & 1 & 0 \\
0 & 0 & 1
\end{array}\right]
$$

Since $M$ is in column-reduced Echelon form already, we have $N=M$. By definition, we have $S_{W}=\{1,3,4\}$, and $B_{W}$ consists of columns of $M$. Moreover, $\mathrm{PBW}_{W}$ consists of columns matrices of the following form (see Remark 3.4):

$$
\left[\begin{array}{lll}
1 & 0 & 0 \\
2 & 0 & 0 \\
0 & 1 & 0 \\
0 & 0 & 1
\end{array}\right]\left[\begin{array}{lll}
1 & 0 & 0 \\
* & 1 & 0 \\
* & * & 1
\end{array}\right]
$$

We have a partial order $\prec$ on subsets of $[n]$ given by $S_{1} \prec S_{2}$ if $\max S_{1}<\min S_{2}$. We now list some crucial properties of our construction above:

(P1) If $W_{1} \oplus W_{2}$ is a summand of $K^{n}$ and $S_{W_{1}} \prec S_{W_{2}}$, then $S_{W_{1} \oplus W_{2}}=S_{W_{1}} \sqcup S_{W_{2}}$.

(P2) If $W_{1} \oplus W_{2}$ is a summand of $K^{n}$ and $S_{W_{1}} \prec S_{W_{2}}$, then the multiplication

$$
\mathbf{S t}\left(W_{1}\right) \otimes \mathbf{S t}\left(W_{2}\right) \rightarrow \mathbf{S t}\left(W_{1} \oplus W_{2}\right)
$$

takes $\mathrm{PBW}_{W_{1}} \times \mathrm{PBW}_{W_{2}}$ inside $\mathrm{PBW}_{W_{1} \oplus W_{2}}$.

(P3) Given a partition $S_{1} \sqcup S_{2}$ of $S_{W}$ with $S_{1} \prec S_{2}$, there is a natural map

$$
\mathrm{PBW}_{W} \rightarrow \coprod_{W_{1} \oplus W_{2}=W} \mathrm{PBW}_{W_{1}} \times \mathrm{PBW}_{W_{2}}
$$


which we now describe. Suppose the ordered basis is $B_{W}=\left(v_{1}, \ldots, v_{d}\right)$. Then every element in $\mathrm{PBW}_{W}$ can be written uniquely in the form $\left[g v_{1}, g v_{2}, \ldots, g v_{d}\right]$ for some $g \in \mathbf{U}_{d}$. Suppose the sizes of $S_{1}$ and $S_{2}$ are $d_{1}$ and $d_{2}$ respectively, then the claimed natural map is given by

$$
\left[g v_{1}, g v_{2}, \ldots, g v_{d}\right] \mapsto\left(\left[g v_{1}, g v_{2}, \ldots, g v_{d_{1}}\right],\left[g v_{d_{1}+1}, \ldots, g v_{d}\right]\right)
$$

The corresponding $W_{1}$ and $W_{2}$ are given by the spans of $\left\{g v_{1}, g v_{2}, \ldots, g v_{d_{1}}\right\}$ and $\left\{g v_{d_{1}+1}, \ldots, g v_{d}\right\}$, respectively. Note that then $S_{W_{1}}=S_{1}$ and $S_{W_{2}}=S_{2}$.

Remark 3.5. We restrict to the case when $R=K$ is a field because the property (P3) as above does not have an analogue for more general rings. For example, the issue when $R=\mathbb{Z}$ is the existence of a finite-rank free abelian group $W$ together with a decomposition $A \oplus B$ of $W \otimes_{\mathbb{Z}} \mathbb{Q}$ such that $(W \cap A) \oplus(W \cap B) \neq W$.

We now follow Brunetti-Ciampella's argument [BC07, Theorem 2.5] which in turn is based on an argument of Priddy [Pri70, Theorem 5.3] to complete our proof of Koszulness with Proposition 3.6.

Proposition 3.6. $\mathrm{H}_{s}\left(\overline{\mathcal{B}}_{*}^{n}(\mathbf{S t})\right)=0$ for $s \neq n$.

Proof. Because $\overline{\mathcal{B}}_{*}^{n}(\mathbf{S t})$ is supported in homological degrees $\leq n$, it is enough to prove that $\mathrm{H}_{s}\left(\overline{\mathcal{B}}_{*}^{n}(\mathbf{S t})\right)=0$ for $s<n$.

We define a k-linear map $\Phi: \overline{\mathcal{B}}_{s}^{n}(\mathbf{S t}) \rightarrow \overline{\mathcal{B}}_{s+1}^{n}(\mathbf{S t})$ on every $x=\left[a_{W_{1}}|\cdots| a_{W_{s}}\right]$ with $a_{W_{j}} \in \mathrm{PBW}_{W_{j}}$ for each $j$ (such elements form a $\mathbf{k}$-basis of $\overline{\mathcal{B}}_{s}^{n}(\mathbf{S t})$ ) as follows:

(a) Set $k$ to be the smallest index such that rank $W_{k}>1$. (If such $k$ does not exist, $\Phi(x)=0$ automatically because $x$ is in top degree.) We call this the widening index of $x$.

(b) If $S_{W_{j}} \prec S_{W_{j+1}}$, we call $j$ an orderpreserving index of $x$. If there is an orderpreserving index $j$ strictly smaller than $k$, set $\Phi(x)=0$. Example: Suppose $n=4$, and let $x=$ $\left[a_{W_{1}}\left|a_{W_{2}}\right| a_{W_{3}}\right] \in \overline{\mathcal{B}}_{3}^{n}(\mathbf{S t})$ be given by

$$
x=\left[\left[\begin{array}{l}
1 \\
0 \\
0 \\
0
\end{array}\right]\left|\left[\begin{array}{l}
0 \\
1 \\
0 \\
0
\end{array}\right]\right|\left[\begin{array}{ll}
0 & 0 \\
0 & 0 \\
1 & 0 \\
0 & 1
\end{array}\right]\right] .
$$

Then $k=3$, and $S_{W_{1}}=\{1\} \prec\{2\}=S_{W_{2}}$. So $j=1$ is an orderpreserving index strictly smaller than $k$.

(c) Otherwise, let $m=\min S_{W_{k}}$ and $S$ be the complement of $m$ in $S_{W_{k}}$.

(d) By property (P3), the partition $\{m\} \sqcup S$ gives rise to a map

$$
\mathrm{PBW}_{W_{k}} \rightarrow \coprod_{W \oplus W^{\prime}=W_{k}} \mathrm{PBW}_{W} \times \mathrm{PBW}_{W^{\prime}}
$$

Let $\left(b, b^{\prime}\right)$ be the image of $a_{W_{k}}$ under this map. 
(e) Set $\Phi(x)=(-1)^{k-1}\left[a_{W_{1}}|\cdots| a_{W_{k-1}}|b| b^{\prime}\left|a_{W_{k+1}}\right| \cdots \mid a_{W_{s}}\right]$. Example: Suppose $n=4$, and let $x=\left[a_{W_{1}}\left|a_{W_{2}}\right| a_{W_{3}}\right] \in \overline{\mathcal{B}}_{3}^{n}(\mathbf{S t})$ be given by

$$
x=\left[\left[\begin{array}{l}
0 \\
0 \\
0 \\
1
\end{array}\right]\left|\left[\begin{array}{l}
0 \\
0 \\
1 \\
0
\end{array}\right]\right|\left[\begin{array}{ll}
1 & 0 \\
6 & 1 \\
3 & 0 \\
5 & 0
\end{array}\right]\right] .
$$

Then $k=3, S_{W_{1}}=\{4\}, S_{W_{2}}=\{3\}$, and $S_{W_{3}}=\{1,2\}$. So $m=1$, and $S=\{2\}$. In this case $\Phi(x)$ is given by

$$
\Phi(x)=(-1)^{2}\left[\left[\begin{array}{l}
0 \\
0 \\
0 \\
1
\end{array}\right]\left|\left[\begin{array}{l}
0 \\
0 \\
1 \\
0
\end{array}\right]\right|\left[\begin{array}{l}
1 \\
6 \\
3 \\
5
\end{array}\right] \mid\left[\begin{array}{l}
0 \\
1 \\
0 \\
0
\end{array}\right]\right] .
$$

Let us furthermore define a filtration on $\overline{\mathcal{B}}_{s}^{n}(\mathbf{S t})$ :

(a) To every basis element $x=\left[a_{W_{1}}|\cdots| a_{W_{s}}\right]$ with $a_{W_{j}} \in \mathrm{PBW}_{W_{j}}$ we associate a word $w_{x}$ in $[n]^{n}$ by concatenating $S_{W_{1}}, \ldots, S_{W_{s}}$, where the elements of $S_{W_{j}}$ are ordered as natural numbers.

(b) Let $w=\left(i_{1}, \ldots, i_{n}\right)$ be a sequence in $[n]^{n}$. We say that a pair $(\alpha, \beta)$ such that $\alpha<\beta$ is an inversion for $w$ if $i_{\alpha} \geq i_{\beta}$. Define a quasi order $<$ on $[n]^{n}$ by $w<w^{\prime}$ if $w$ has more inversions than $w^{\prime}$.

Note that there is a unique maximal element in $[n]^{n}$ given by $(1,2, \ldots, n)$ which corresponds, for example, to $x=\left[\mathbf{e}_{1}\left|\mathbf{e}_{2}\right| \ldots \mid \mathbf{e}_{n}\right]$ where $\mathbf{e}_{1}, \ldots, \mathbf{e}_{n}$ is the coordinate basis of $K^{n}$ (or to any basis element $x$ which is lower triangular with the notation as in the example above). On the other hand, if $x=\left[\mathbf{e}_{n}\left|\mathbf{e}_{n}+\mathbf{e}_{1}\right| \mathbf{e}_{n}+\mathbf{e}_{2}|\ldots| \mathbf{e}_{n}+\mathbf{e}_{n-1}\right]$ then $w_{x}=(n, n, \ldots, n)$ which is a minimal element in $[n]^{n}$. Intuitively, $w_{x}>w_{y}$ if $x$ is closer to being a lower triangular matrix compared to $y$.

(c) Let $F_{\leq \alpha} \overline{\mathcal{B}}_{s}^{n}(\mathbf{S t})$ be generated by all basis elements $x$ such that the number of inversions in $x$ is at most $\alpha$.

For an element $y \in \overline{\mathcal{B}}_{s}^{n}(\mathbf{S t})$ we say that $w_{y}>w$ if $y$ can be written as a sum of basis elements $x$ (as in (a) above) satisfying $w_{x}>w$. A crucial property of this order is the following. Let $x=\left[a_{W_{1}}|\cdots| a_{W_{s}}\right]$ be a basis element and let $y=\left[a_{W_{1}}|\cdots| a_{W_{j-1}}\left|a_{W_{j}} a_{W_{j+1}}\right| a_{W_{j+2}}|\cdots| a_{W_{s}}\right]$, where $a_{W_{j}} a_{W_{j+1}}$ is the image of $a_{W_{j}} \otimes a_{W_{j+1}}$ under the map $\mathbf{S t}\left(W_{j}\right) \otimes \mathbf{S t}\left(W_{j+1}\right) \rightarrow \mathbf{S t}\left(W_{1} \oplus W_{2}\right)$. Then we have $w_{x} \leq w_{y}$ and the equality holds if and only if $S_{W_{j}} \prec S_{W_{j+1}}$ (if and only if $y$ is a basis element). In particular,

$$
0=F_{\leq-1} \overline{\mathcal{B}}_{*}^{n}(\mathbf{S t}) \subset F_{\leq 0} \overline{\mathcal{B}}_{*}^{n}(\mathbf{S t}) \subset F_{\leq 1} \overline{\mathcal{B}}_{*}^{n}(\mathbf{S t}) \subset \ldots \subset F_{\leq\left(\begin{array}{l}
n \\
2
\end{array}\right)} \overline{\mathcal{B}}_{*}^{n}(\mathbf{S t})=\overline{\mathcal{B}}_{*}^{n}(\mathbf{S t})
$$

defines a finite increasing filtration on the chain complex $\overline{\mathcal{B}}_{*}^{n}(\mathbf{S t})$. We also note here that $w_{\Phi(x)}=w_{x}$ for every basis element $x$ for which $\Phi(x)$ is nonzero.

In the remainder of the proof, we will verify that $\partial \Phi+\Phi \partial-$ id sends $F_{\leq \alpha} \overline{\mathcal{B}}_{s}^{n}(\mathbf{S t})$ to $F_{<\alpha} \overline{\mathcal{B}}_{s}^{n}(\mathbf{S t})$ for all $s<n$. This then shows that

$$
\mathrm{H}_{s}\left(F_{\leq \alpha} \overline{\mathcal{B}}_{*}^{n}(\mathbf{S t}) / F_{<\alpha} \overline{\mathcal{B}}_{*}^{n}(\mathbf{S t})\right)=0 \quad \text { for } s<n,
$$


because every cycle

$$
c=(\partial \Phi+\Phi \partial)(c)=\partial(\Phi(c))
$$

in degree less than $n$ is also a boundary. By induction on the filtration, we deduce that $\mathrm{H}_{s}\left(\overline{\mathcal{B}}_{*}^{n}(\mathbf{S t})\right)=0$ for $s<n$. We prove the claim by a complete case study. Let $x=\left[a_{W_{1}}|\cdots| a_{W_{s}}\right]$ with $a_{W_{j}} \in \mathrm{PBW}_{W_{j}}$ and $s<n$. Set $k$ to be the smallest index such that rank $W_{k}>1$.

Case 1: Assume there is a $j<k$ such that $S_{W_{j}} \prec S_{W_{j+1}}$. Also let $j_{0}$ be the smallest orderpreserving index. Then $(\partial \Phi+\Phi \partial)(x)=\Phi \partial(x)$. Let $y_{i}=\left[a_{W_{1}}|\ldots| a_{W_{i}} a_{W_{i+1}}|\ldots| a_{W_{s}}\right]$, so that

$$
\partial(x)=\sum_{i=1}^{s-1}(-1)^{i-1} y_{i} .
$$

First we see that $\Phi\left(y_{j_{0}}\right)=x$, because $j_{0}$ is the widening index of $y_{j_{0}}$ and there is no orderpreserving indices smaller than $j_{0}$. For $i<j_{0}$, we get that $w_{y_{i}}>w_{x}$, because $i$ is not an orderpreserving index of $x$. Hence $w_{\Phi\left(y_{i}\right)}=w_{y_{i}}>w_{x}$. If $i=j_{0}+1$, we have two cases. If $j_{0}$ is an orderpreserving index of $y_{j_{0}+1}$, then $\Phi\left(y_{j_{0}+1}\right)=0$ because its widening index is $j_{0}+1$. It is possible that $j_{0}$ is not an orderpreserving index if $S_{W_{j_{0}}} \nprec S_{W_{j_{0}+2}}$, but then $j_{0}+1$ cannot be orderpreserving and $w_{\Phi\left(y_{j_{0}+1}\right)}=w_{y_{j_{0}+1}}>w_{x}$. Finally, if $i \geq j_{0}+2, j_{0}$ is orderpreserving and smaller than the widening index of $y_{i}$. Therefore $\Phi\left(y_{i}\right)=0$.

Case 2: Assume there is no $j<k$ such that $S_{W_{j}} \prec S_{W_{j+1}}$. Let $y_{i}=\left[a_{W_{1}}|\ldots| a_{W_{i}} a_{W_{i+1}}|\ldots| a_{W_{s}}\right]$. Let us write $\Phi(x)=(-1)^{k-1}\left[a_{W_{1}}|\cdots| a_{W_{k-1}}\left|a_{W_{k}^{\prime}}\right| a_{W_{k}^{\prime \prime}}\left|a_{W_{k+1}}\right| \cdots \mid a_{W_{s}}\right]$ as in its definition, that is, $\left(a_{W_{k}^{\prime}}, a_{W_{k}^{\prime \prime}}\right)$ is the image of $a_{W_{k}}$ under the map

$$
\mathrm{PBW}_{W_{k}} \rightarrow \coprod_{W_{k}^{\prime} \oplus W_{k}^{\prime \prime}=W_{k}} \mathrm{PBW}_{W_{k}^{\prime}} \times \mathrm{PBW}_{W_{k}^{\prime \prime}}
$$

We now define

$$
z_{i}= \begin{cases}{\left[a_{W_{1}}|\ldots| a_{W_{i}} a_{W_{i+1}}|\cdots| a_{W_{k-1}}\left|a_{W_{k}^{\prime}}\right| a_{W_{k}^{\prime \prime}}\left|a_{W_{k+1}}\right| \cdots \mid a_{W_{s}}\right]} & \text { if } i<k-1, \\ {\left[a_{W_{1}}|\ldots| a_{W_{i}} a_{W_{i+1}}|\cdots| a_{W_{k-1}} a_{W_{k}^{\prime}}\left|a_{W_{k}^{\prime \prime}}\right| a_{W_{k+1}}|\cdots| a_{W_{s}}\right]} & \text { if } i=k-1, \\ {\left[a_{W_{1}}|\cdots| a_{W_{k-1}}\left|a_{W_{k}^{\prime}} a_{W_{k}^{\prime \prime}}\right| a_{W_{k+1}}|\cdots| a_{W_{s}}\right]} & \text { if } i=k, \\ {\left[a_{W_{1}}|\cdots| a_{W_{k-1}}\left|a_{W_{k}^{\prime}}\right| a_{W_{k}^{\prime \prime}} a_{W_{k+1}}|\cdots| a_{W_{s}}\right]} & \text { if } i=k+1 \\ {\left[a_{W_{1}}|\ldots| a_{W_{k-1}}\left|a_{W_{k}^{\prime}}\right| a_{W_{k}^{\prime \prime}}\left|a_{W_{k+1}}\right| \cdots\left|a_{W_{i-1}} a_{W_{i}}\right| \cdots \mid a_{W_{s}}\right]} & \text { if } i>k+1 .\end{cases}
$$

Then

$$
\partial(x)=\sum_{i=1}^{s-1}(-1)^{i-1} y_{i} \quad \text { and } \quad \partial \Phi(x)=\sum_{i=1}^{s}(-1)^{i-1}(-1)^{k-1} z_{i} .
$$

Then we have the following:

- For $i<k, w_{\Phi\left(y_{i}\right)}=w_{y_{i}}$ is larger than $w_{x}$ as $S_{W_{i}} \nprec S_{W_{i+1}}(i$ is not an orderpreserving index of $x$ ).

- For $i<k-1, w_{z_{i}}$ is larger than $w_{x}$ again because $S_{W_{i}} \nprec S_{W_{i+1}}(i$ is not an orderpreserving index of $x$ ).

- $w_{z_{k-1}}$ is larger than $w_{x}$ as $S_{W_{k-1}} \nprec S_{W_{k}^{\prime}}$ (recall that $S_{W_{k}^{\prime}}=\min S_{W_{k}}$ and $k-1$ is not an orderpreserving index for $x$ ).

- $z_{k}=x$ as $S_{W_{k}^{\prime}} \prec S_{W_{k}^{\prime \prime}}$ by construction (in other words, $a_{W_{k}^{\prime}} a_{W_{k}^{\prime \prime}}=a_{W_{k}}$ is already a basis element in $\left.\mathbf{S t}\left(W_{k}\right)\right)$. 
- If $z_{k+1}$ and $\Phi\left(y_{k}\right)$ do not cancel, $k$ cannot be an orderpreserving index of $x$, which implies that both $w_{\Phi\left(y_{k}\right)}=w_{y_{k}}$ and $w_{z_{k+1}}$ are larger than $w_{x}$.

- $\Phi\left(y_{i}\right)$ cancels with $z_{i+1}$ in $(\partial \Phi+\Phi \partial)(x)$ if $i>k$. To see this, just note that the widening index for each term in $y_{i}$ expressed in the basis (a) has widening index $k$ and orderpreserving index $\geq k$, and so we have

$$
\Phi\left(y_{i}\right)=(-1)^{k-1}\left[a_{W_{1}}|\ldots| a_{W_{k-1}}\left|a_{W_{k}^{\prime}}\right| a_{W_{k}^{\prime \prime}}\left|a_{W_{k+1}}\right| \cdots\left|a_{W_{i}} a_{W_{i+1}}\right| \cdots \mid a_{W_{s}}\right]
$$

even if $a_{W_{i}} a_{W_{i+1}}$ is not a basis element.

This finishes the case study and the proof.

The proposition above immediately implies Theorem 3.2.

Question 3.7. Can one prove a version of Theorem 3.2 when $R$ is not a field, for example, when $R=\mathbb{Z}$ ? A version of Theorem 3.2 or even Theorem 4.2 for $R=\mathbb{Z}$ would establish the Church-FarbPutman conjecture [CFP14, Conjecture 2] on vanishing of the high-dimensional rational cohomology of $\mathbf{S L}_{n}(\mathbb{Z})$ (except with a slightly worse range).

3.3. Koszul resolutions. Let $A$ be an augmented monoid in $\operatorname{Mod}_{\mathrm{VB}}$ such that $A(X)$ is $\mathbf{k}$-flat for each $X$. Then the functor $-\otimes A$ is exact and is the left adjoint to the restriction functor $\operatorname{Mod}_{A} \rightarrow \operatorname{Mod}_{\mathrm{VB}}$. This adjunction follows from the Yoneda lemma as an $A$-module is the same as a functor $\mathcal{C} \rightarrow \operatorname{Mod}_{\mathbf{k}}$ where $\mathcal{C}$ is the category whose objects are finite-rank free $R$-modules and whose morphisms $X \rightarrow Y$ are triples $(f, C, a)$ where $f$ is an injection of $R$ modules such that $Y=X \oplus C$, and $a \in A(C)$. Since the restriction functor is exact, we see that the $A$-module $V \otimes A$ is projective for any projective VB-module $V$. It follows that the $A$-module $V \otimes A$ is $-\otimes_{A} \mathbf{k}$-acyclic for any VB-module $V$. If $A$ is Koszul, then $\operatorname{Tor}_{i}^{A}(\mathbf{k}, \mathbf{k})$ is concentrated in degree $i$ (for each $i \geq 0$ ). So, by a dimension shifting argument, one can construct a linear resolution

$$
V_{*} \otimes A \rightarrow \mathbf{k} \rightarrow 0
$$

such that $V_{i}$ is a VB-module concentrated only in degree $i$. The converse is also true, that is, if such a resolution exists then $A$ is Koszul. This resolution is called the Koszul resolution. In fact, we can take $V_{i}=\operatorname{Tor}_{i}^{A}(\mathbf{k}, \mathbf{k})$ in the Koszul resolution. As an example, let $\operatorname{reg}_{1}$ be the VB-module such that $\operatorname{reg}_{1}(n)=0$ for $n \neq 1$, and $\operatorname{reg}_{1}(1)$ is the regular representation of $\mathbf{G L}_{1}$. The following sequence is clearly exact:

$$
0 \rightarrow \mathrm{T}\left(\mathrm{reg}_{1}\right) \otimes \operatorname{reg}_{1} \rightarrow \mathrm{T}\left(\mathrm{reg}_{1}\right) \rightarrow \mathbf{k} \rightarrow 0
$$

In particular, $\operatorname{Tor}_{1}^{\mathrm{T}\left(\mathrm{reg}_{1}\right)}(\mathbf{k}, \mathbf{k})=\operatorname{reg}_{1}$ and $\operatorname{Tor}_{i}^{\mathrm{T}\left(E^{\prime}\right)}(\mathbf{k}, \mathbf{k})=0$ for $i>1$. This proves the following result.

Proposition 3.8. If $R$ is a PID, then the tensor algebra $\mathrm{T}\left(\mathrm{reg}_{1}\right)$ is Koszul.

The Koszul resolution for exterior algebras is well-known. In particular, the Koszul resolution for the apartment monoid $\mathbf{A}=\bigwedge\left(\operatorname{triv}_{1}\right)$ is given by the following:

$$
\operatorname{Sym}^{*}\left(\operatorname{triv}_{1}\right) \otimes \mathbf{A} \rightarrow \mathbf{k} \rightarrow 0 .
$$


This is equivalent to $\operatorname{Tor}_{*}^{\mathbf{A}}(\mathbf{k}, \mathbf{k})=\operatorname{Sym}^{*}\left(\operatorname{triv}_{1}\right)$. Let $M$ be an $\mathbf{A}$-module. Since $\operatorname{Sym}^{*}\left(\operatorname{triv}_{1}\right) \otimes \mathbf{A}$ is a flat $\mathbf{A}$-resolution of $\mathbf{k}$, one can calculate $\operatorname{Tor}_{i}^{\mathbf{A}}(\mathbf{k}, M)$ using the Koszul resolution. In other words, we have

$$
\operatorname{Tor}_{i}^{\mathbf{A}}(\mathbf{k}, M)=\mathrm{H}_{i}\left(\operatorname{Sym}^{*}\left(\operatorname{triv}_{1}\right) \otimes M\right) .
$$

We will need the following calculation later.

Lemma 3.9. Let $K=\mathbb{F}_{q}$ be a finite field of size $q$, and let $\mathbf{A}$ and $\mathbf{S t}$ be the apartment and the Steinberg monoids in $\operatorname{Mod}_{\mathrm{VB}_{\mathbb{F}_{q}}}$. If $\mathbf{k}$ is a field, then

$$
\operatorname{dim}_{\mathbf{k}} \operatorname{Tor}_{2}^{\mathbf{A}}(\mathbf{k}, \mathbf{S t})\left(\mathbb{F}_{q}^{4}\right) \leq \frac{\left(q^{4}-1\right)\left(q^{3}-1\right) q^{6}}{2(q-1)^{2}}
$$

Proof. By the previous paragraph, we have

$$
\operatorname{dim}_{\mathbf{k}} \operatorname{Tor}_{2}^{\mathbf{A}}(\mathbf{k}, \mathbf{S t})\left(\mathbb{F}_{q}^{4}\right) \leq \operatorname{dim}_{\mathbf{k}}\left(\operatorname{Sym}^{2}\left(\operatorname{triv}_{1}\right) \otimes \mathbf{S t}\right)\left(\mathbb{F}_{q}^{4}\right)
$$

Now note that

$$
\begin{aligned}
\operatorname{dim}_{\mathbf{k}} \operatorname{Sym}^{2}\left(\operatorname{triv}_{1}\right) & =\frac{\left|\mathbf{G L}_{2}\left(\mathbb{F}_{q}\right)\right|}{\left|\mathbf{G L}_{1}\left(\mathbb{F}_{q}\right)\right|^{2}\left|S_{2}\right|} \\
\operatorname{dim}_{\mathbf{k}} \operatorname{St}\left(\mathbb{F}_{q}^{2}\right) & =q,
\end{aligned}
$$

and so

$$
\operatorname{dim}_{\mathbf{k}}\left(\operatorname{Sym}^{2}\left(\operatorname{triv}_{1}\right) \otimes \mathbf{S t}\right)\left(\mathbb{F}_{q}^{4}\right)=\frac{\left|\mathbf{G L}_{4}\left(\mathbb{F}_{q}\right)\right|}{\left|\mathbf{G L}_{2}\left(\mathbb{F}_{q}\right)\right|^{2}} \frac{\left|\mathbf{G L}_{2}\left(\mathbb{F}_{q}\right)\right|}{\left|\mathbf{G L}_{1}\left(\mathbb{F}_{q}\right)\right|^{2}\left|S_{2}\right|} q=\frac{\left(q^{4}-1\right)\left(q^{3}-1\right) q^{6}}{2(q-1)^{2}}
$$

This completes the proof.

We do not know an explicit description of the Koszul resolution for the Steinberg monoid, but it is possible to compute its dimension.

Proposition 3.10. Let $K=\mathbb{F}_{q}$ be a finite field of size $q$, and let $\mathbf{S t}$ be the Steinberg monoid in $\operatorname{Mod}_{\mathrm{VB}_{\mathbb{F}_{q}}}$. If $\mathbf{k}$ is a field, then $\operatorname{dim}_{\mathbf{k}} \operatorname{Tor}_{n}^{\mathbf{S t}}(\mathbf{k}, \mathbf{k})=q^{n^{2}-n}$.

Proof. Let $T_{n}$ denote $\operatorname{dim}_{\mathbf{k}} \operatorname{Tor}_{n}^{\mathbf{S t}}(\mathbf{k}, \mathbf{k})$, and let $\left[\begin{array}{c}n \\ i\end{array}\right]_{q}$ denote the $q$-binomial coefficient. It is clear that $T_{0}=1$, and we have

$$
\frac{\left|\mathbf{G L}_{n}\left(\mathbb{F}_{q}\right)\right|}{\left|\mathbf{G L}_{i}\left(\mathbb{F}_{q}\right)\right|\left|\mathbf{G L}_{n-i}\left(\mathbb{F}_{q}\right)\right|}=\left[\begin{array}{c}
n \\
i
\end{array}\right]_{q} q^{i(n-i)} .
$$

Since $\mathbf{S t}$ is Koszul (Theorem 3.2), we have a Koszul resolution given by $\mathbf{S t} \otimes \operatorname{Tor}_{*}^{\mathbf{S t}}(\mathbf{k}, \mathbf{k}) \rightarrow \mathbf{k} \rightarrow 0$. So $T_{n}$ satisfy the recursion

$$
T_{n}=\sum_{i=1}^{n}(-1)^{i}\left[\begin{array}{c}
n \\
i
\end{array}\right]_{q} q^{i(n-i)} q^{\left(\begin{array}{c}
i \\
2
\end{array}\right)} T_{n-i}
$$

By induction, it suffices to verify that

$$
q^{n^{2}-n}=\sum_{i=1}^{n}(-1)^{i}\left[\begin{array}{l}
n \\
i
\end{array}\right]_{q} q^{i(n-i)} q^{\left(\begin{array}{c}
i \\
2
\end{array}\right)} q^{(n-i)^{2}-(n-i)},
$$

which is equivalent, after cancelling $q^{\left(\begin{array}{l}n \\ 2\end{array}\right)}$ from both sides, to

$$
q^{\left(\begin{array}{c}
n \\
2
\end{array}\right)}=\sum_{i=1}^{n}(-1)^{i}\left[\begin{array}{c}
n \\
i
\end{array}\right]_{q} q^{\left(\begin{array}{c}
n-i \\
2
\end{array}\right)}
$$


This follows from [Nag19, Lemma 5.9], completing the proof.

Question 3.11. Can one give a conceptual description of the Koszul dual of the Steinberg monoid, or explicitly describe the cycles in $\overline{\mathcal{B}}_{n}^{n}(\mathbf{S t})$ ?

\section{Finiteness properties of Resolutions of the Steinberg module}

In this section, we study the groups $\operatorname{Tor}_{n}^{\mathbf{A}}(\mathbf{S t}, \mathbf{k})$.

4.1. Presentation of the Steinberg monoid as a module over the apartment monoid. We reinterpret the Bykovskii presentation [Byk03] and the presentation appearing in Theorem 2.8 as presentations of $\mathbf{S t}$ as an $\mathbf{A}$-module and use this to prove the following.

Proposition 4.1. For $R=\mathbb{Z}$ or a field, we have $\operatorname{deg} \operatorname{Tor}_{i}^{\mathbf{A}}(\mathbf{k}, \mathbf{S t})=2 i$ for $i=0,1$.

Proof. The Bykovskii presentation [Byk03] (here Theorem 2.5) in the case of the integers and Theorem 2.8 in the case of fields implies we have an exact sequence

$$
\mathbf{A} \otimes M \rightarrow \mathbf{A} \rightarrow \mathbf{S t} \rightarrow 0
$$

of A-modules where $M$ is a VB-module supported only in degree 2 and is given by

$$
M(X)=\left\langle\left[v_{1}, v_{2}\right]-\left[v_{0}, v_{2}\right]+\left[v_{0}, v_{1}\right] \mid v_{0}=v_{1}+v_{2}\right\rangle \subset \mathbf{A}(X)
$$

where $X$ is a free $R$-module of rank 2 . The assertion is immediate from this partial resolution.

4.2. Higher syzygies. In the previous subsection, we showed that for $R=\mathbb{Z}$ or a field, we have $\operatorname{deg} \operatorname{Tor}_{i}^{\mathbf{A}}(\mathbf{k}, \mathbf{S t})=2 i$ for $i=0,1$. In this subsection, we prove that if $R$ is a field, we may drop the restriction on $i$. Fix a field $K$. Throughout this subsection we shall assume that $R=K$. Let $\mathbf{S t}$ and $\mathbf{A}$ be the Steinberg and the apartment monoids in $\operatorname{Mod}_{\mathrm{VB}}=\operatorname{Mod}_{\mathrm{VB}_{K}}$, respectively. The main theorem of this section is the following.

Theorem 4.2. For $K$ a field, $\operatorname{deg} \operatorname{Tor}_{i}^{\mathbf{A}}(\mathbf{k}, \mathbf{S t}) \leq 2 i$ for $i \geq 0$.

Basic properties of Tor $_{*}$ and the theorem above implies the following:

Corollary 4.3 (Existence of an improved resolution). There is a resolution of the form $\mathbf{A} \otimes V_{*} \rightarrow$ $\mathrm{St} \rightarrow 0$ where $V_{i}$ is a $\mathrm{VB}_{K}$-module supported in degrees $\leq 2 i$.

It would be interesting to find an explicit resolution with the above properties. Below we prove a result on surjections of Koszul monoids (Proposition 4.6) and combine it with the Koszulness results from the previous section to prove Theorem 4.2 .

Lemma 4.4. Suppose $A$ is a Koszul monoid in $\left(\operatorname{Mod}_{\mathrm{VB}}, \otimes\right)$. If $M$ is an A-module supported in degrees $\leq d$, then $\operatorname{deg} \operatorname{Tor}_{i}^{A}(M, \mathbf{k}) \leq i+d$.

Proof. Let $A \otimes V_{*} \rightarrow \mathbf{k} \rightarrow 0$ be the Koszul resolution. In particular, $V_{i}$ is concentrated in degree $i$. Now note that $\operatorname{Tor}_{i}^{A}(M, \mathbf{k})=\mathrm{H}_{i}\left(M \otimes V_{*}\right)$. Since $V_{i}$ is concentrated in degree $i$ and $M$ is supported in degrees $\leq d$, we see that $M \otimes V_{*}$ is supported in degrees $\leq(i+d)$. This finishes the proof. 
Lemma 4.5. Let $A \rightarrow B \rightarrow \mathbf{k}$ be surjections of (skew) commutative monoids in (Mod $\left.\mathrm{VB}_{\mathrm{B}}, \otimes\right)$. Assume that the kernels $A_{+}$and $B_{+}$of the surjections $A \rightarrow \mathbf{k}$ and $B \rightarrow \mathbf{k}$ are supported in degrees $>0$. Then

$$
\operatorname{Tor}_{*}^{A}(\mathbf{k}, B) \otimes_{B} \mathbf{k}=\operatorname{Tor}_{*}^{A}(\mathbf{k}, B) .
$$

Proof. Let $P_{*} \rightarrow \mathbf{k}$ and $Q_{*} \rightarrow B$ be free $A$-resolutions. Then $\operatorname{Tor}_{i}^{A}(\mathbf{k}, B)=\mathrm{H}_{i}\left(\mathbf{k} \otimes_{A} Q_{*}\right)$. Since $A_{+}$ acts trivially on $\mathbf{k} \otimes_{A} Q_{i}=Q_{i} /\left(A_{+} Q_{i}\right)$, we conclude that $A_{+}$acts trivially on $\operatorname{Tor}_{i}^{A}(\mathbf{k}, B)$. However, $A_{+}$acts via its image $B_{+}$on $\operatorname{Tor}_{i}^{A}(\mathbf{k}, B)=\mathrm{H}_{i}\left(P_{*} \otimes_{A} B\right)$. Thus $B_{+}$acts trivially on $\operatorname{Tor}_{i}^{A}(\mathbf{k}, B)$. The assertion is immediate from this.

The following result is inspired by Chardin-Symonds [CS16, §5].

Proposition 4.6. Let $A \rightarrow B \rightarrow \mathbf{k}$ be surjections of (skew) commutative monoids in (Mod $\left.\mathrm{VB}_{\mathrm{B}}, \otimes\right)$. Assume that the kernels $A_{+}$and $B_{+}$of the surjections $A \rightarrow \mathbf{k}$ and $B \rightarrow \mathbf{k}$ are supported in degrees $>0$. If $A$ and $B$ are Koszul, then

$$
\operatorname{deg} \operatorname{Tor}_{i}^{A}(\mathbf{k}, B) \leq 2 i \quad \text { for all } i \geq 0 .
$$

Proof. Denote $\operatorname{deg} \operatorname{Tor}_{i}^{A}(\mathbf{k}, B)$ by $s_{i}$. We prove by induction on $i$ that $s_{i} \leq 2 i$. The base case $i=0$ is trivial. Let $i>0$. Consider the base change spectral sequence

$$
E_{a b}^{2}=\operatorname{Tor}_{a}^{B}\left(\operatorname{Tor}_{b}^{A}(\mathbf{k}, B), \mathbf{k}\right) \Longrightarrow \operatorname{Tor}_{a+b}^{A}(\mathbf{k}, \mathbf{k}) .
$$

Let $t_{i}^{A}$ denote $\operatorname{deg} \operatorname{Tor}_{i}^{A}(\mathbf{k}, \mathbf{k})$. By Koszulness of $A$, we know that $t_{i}^{A} \leq i$. Now suppose $b<i$. By induction, $s_{b} \leq 2 b$. By Lemma 4.4 and Koszulness of $B$, we have

$$
\operatorname{deg} E_{a b}^{2} \leq a+s_{b} \leq a+2 b .
$$

The spectral sequence now implies that

$$
\operatorname{deg} E_{0, i}^{2} \leq \max \left(t_{i}^{A},_{a+b=i+1, b<i} \operatorname{deg} E_{a b}^{2}\right) \leq 2 i .
$$

Now note that $E_{0, i}^{2}=\operatorname{Tor}_{0}^{B}\left(\operatorname{Tor}_{i}^{A}(\mathbf{k}, B), \mathbf{k}\right)=\operatorname{Tor}_{i}^{A}(\mathbf{k}, B)$ by Lemma 4.5. Thus $s_{i} \leq 2 i$, completing the proof.

Proof of Theorem 4.2. The theorem follows immediately from the previous proposition and Koszulness of the apartment and the Steinberg monoids (Theorem 3.1 and Theorem 3.2).

\section{Codimension one Cohomology of the level 3 Congruence subgroup}

Let $\Gamma_{n}(p)$ be the level $p$ congruence subgroup of $\mathbf{S L}_{n}(\mathbb{Z})$. In this section, we show that the integral codimension-one cohomology of $\Gamma_{n}(3)$ is representation stable in the sense that the sequence

$$
\left\{\mathrm{H}^{\left(\begin{array}{l}
n \\
2
\end{array}\right)-1}\left(\Gamma_{n}(3)\right)\right\}_{n \geq 0}
$$

of representations is generated in degrees $\leq 4$. In other words, the $\mathbf{G L}_{n}\left(\mathbb{F}_{3}\right)$-equivariant map

$$
\operatorname{Ind}_{\mathbf{G L}_{4}\left(\mathbb{F}_{3}\right)}^{\mathbf{G L}_{n}\left(\mathbb{F}_{3}\right)} \mathrm{H}^{\left(\begin{array}{l}
4 \\
2
\end{array}\right)-1}\left(\Gamma_{4}(3)\right) \rightarrow \mathrm{H}^{\left(\begin{array}{c}
n \\
2
\end{array}\right)-1}\left(\Gamma_{n}(3)\right)
$$

is surjective for $n \geq 4$. We also bound the dimensions of these groups with field coefficients. By Borel-Serre duality [BS73], we have

$$
\mathrm{H}^{\left(\begin{array}{l}
n \\
2
\end{array}\right)-i}\left(\Gamma_{n}(3) ; \mathbf{k}\right)=\mathrm{H}_{i}\left(\Gamma_{n}(3) ; \mathrm{St}_{n}(\mathbb{Z}) \otimes_{\mathbb{Z}} \mathbf{k}\right)
$$


for every coefficient ring $\mathbf{k}$. Thus, it suffices to prove the following representation stability result.

Theorem 5.1. The sequence

$$
\left\{\mathrm{H}_{1}\left(\Gamma_{n}(3) ; \mathrm{St}_{n}(\mathbb{Z}) \otimes_{\mathbb{Z}} \mathbf{k}\right)\right\}_{n \geq 0}
$$

is generated in degrees $\leq 4$. Moreover, if $\mathbf{k}$ is a field and $n \geq 4$ then we have

$$
\operatorname{dim}_{\mathbf{k}} \mathrm{H}_{1}\left(\Gamma_{n}(3) ; \operatorname{St}_{n}(\mathbb{Z}) \otimes_{\mathbb{Z}} \mathbf{k}\right) \leq \frac{3^{\left(\begin{array}{c}
n-4 \\
2
\end{array}\right)}\left|\mathbf{G L}_{n}\left(\mathbb{F}_{3}\right)\right|}{\left|\mathbf{G L}_{n-4}\left(\mathbb{F}_{3}\right)\right|\left|\mathbf{G L}_{4}\left(\mathbb{F}_{3}\right)\right|} \operatorname{dim}_{\mathbf{k}} \mathrm{H}_{1}\left(\Gamma_{4}(3) ; \operatorname{St}_{4}(\mathbb{Z}) \otimes_{\mathbb{Z}} \mathbf{k}\right) .
$$

We begin by explaining our setup and constructing a spectral sequence in §5.1. Our setup is quite similar to that of [Dja] and that of [PS17], but we believe that our spectral sequence is new. In $\$ 5.2$, we use Theorem 4.2 on resolutions of the Steinberg monoid and the spectral sequence of Theorem 5.9 to prove Theorem 5.1 above. We also obtain a rough upper bound on $\operatorname{dim}_{\mathbf{k}} \mathrm{H}_{1}\left(\Gamma_{4}(3) ; \mathrm{St}_{4}(\mathbb{Z}) \otimes_{\mathbb{Z}} \mathbf{k}\right)$ to obtain the following corollary.

Corollary 5.2. Suppose $\mathbf{k}$ is a field and assume that $n \geq 4$. Then

$$
\operatorname{dim}_{\mathbf{k}} \mathrm{H}_{1}\left(\Gamma_{n}(3) ; \mathrm{St}_{n}(\mathbb{Z}) \otimes_{\mathbb{Z}} \mathbf{k}\right) \leq \frac{3^{\left(\begin{array}{c}
n-4 \\
2
\end{array}\right)}\left|\mathbf{G L}_{n}\left(\mathbb{F}_{3}\right)\right|}{\left|\mathbf{G L}_{n-4}\left(\mathbb{F}_{3}\right)\right|\left|\mathbf{G L}_{4}\left(\mathbb{F}_{3}\right)\right|} 227340 .
$$

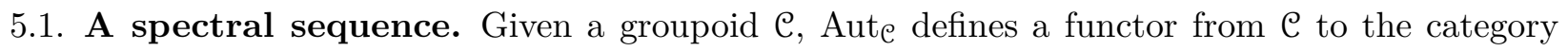
Grp of groups. Using this, we define GL: VB $\rightarrow \operatorname{Grp}$ given by $\mathbf{G L}(X)=\operatorname{Aut}_{\mathrm{VB}}(X)$. Moreover, it has the following properties:

- The following diagram commutes (also see [Dja, Definition 1.1]):

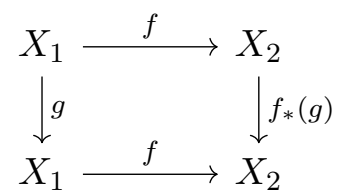

- There is a natural inclusion $\mathbf{G L}\left(X_{1}\right) \times \mathbf{G L}\left(X_{2}\right) \rightarrow \mathbf{G L}\left(X_{1} \oplus X_{2}\right)$ which is functorial in $X_{1}$ and $X_{2}$.

Definition 5.3. Let $\mathcal{G} \subset$ GL be a sub-functor, that is, a functor $\mathcal{G}: \mathrm{VB} \rightarrow$ Grp together with a natural transformation $\iota: \mathcal{G} \rightarrow \mathbf{G L}$ such that $\iota(X): \mathcal{G}(X) \rightarrow \mathbf{G L}(X)$ is an inclusion for each $X$. It is easy to check that $\mathcal{G}(X)$ must be a normal subgroup of $\mathbf{G L}(X)$ for each $X$ under this inclusion. We call $\mathcal{G}$ a strong sub-functor if the following condition holds

$$
\mathcal{G}\left(X_{1} \oplus X_{2}\right) \cap\left(\mathbf{G L}\left(X_{1}\right) \times \mathbf{G L}\left(X_{2}\right)\right)=\mathcal{G}\left(X_{1}\right) \times \mathcal{G}\left(X_{2}\right) .
$$

For a strong sub-functor $\mathcal{G}$, we define $\mathrm{VB}_{\mathbb{Z}} / \mathcal{G}$ to be the category with the same objects as $\mathrm{VB}_{\mathbb{Z}}$ and whose morphisms are $\operatorname{Hom}_{\mathrm{VB}_{\mathbb{Z} / \mathcal{G}}}\left(X_{1}, X_{2}\right)=\mathcal{G}\left(X_{2}\right) \backslash \operatorname{Hom}_{\mathrm{VB}_{\mathbb{Z}}}\left(X_{1}, X_{2}\right)$. There is a natural full and essentially surjective strong symmetric monoidal functor $\Pi_{\mathcal{G}}: \mathrm{VB}_{\mathbb{Z}} \rightarrow \mathrm{VB}_{\mathbb{Z}} / \mathcal{G}$.

Example 5.4. Clearly, GL itself is strong. For a more sophisticated example, consider the natural strong symmetric monoidal functor $\Phi: \mathrm{VB}_{\mathbb{Z}} \rightarrow \mathrm{VB}_{\mathbb{F}_{p}}$ given by $X \mapsto \mathbb{F}_{p} \otimes_{\mathbb{Z}} X$. Then

$$
\Gamma(p)(X)=\operatorname{ker}\left(\operatorname{Aut}_{\mathrm{VB}_{\mathbb{Z}}}(X) \rightarrow \operatorname{Aut}_{\mathrm{VB}}(\Phi(X))\right)
$$

defines a functor $\Gamma(p): \mathrm{VB}_{\mathbb{Z}} \rightarrow$ Grp which is strong. We shall use the usual abbreviation $\Gamma_{n}(p)$ for $\Gamma(p)\left(\mathbb{Z}^{n}\right)$. The functor $\Phi$ factors through $\Pi_{\Gamma(p)}$. The strong monoidal functor $\iota: \operatorname{VB}_{\mathbb{Z}} / \Gamma(p) \rightarrow \operatorname{VB}_{\mathbb{F}_{p}}$ as in the diagram is essentially surjective. Moreover, 


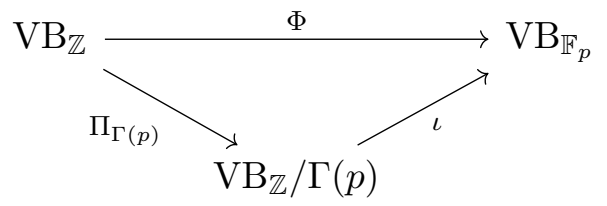

(a) $\iota$ is full if $p \leq 3$.

(b) $\iota$ is faithful if $p \geq 3$.

In particular, $\iota$ is a monoidal equivalence of categories if $p=3$. To see this when $p=3$, just note that we have $\mathbb{Z}^{\times}=\mathbb{F}_{3}^{\times}=\{ \pm 1\}$. Since $\mathbf{G L}_{n}(\mathbb{Z})$ is generated by elementary matrices and their reductions modulo 3 generate $\mathbf{G L}_{n}\left(\mathbb{F}_{3}\right)$, it follows that there is a natural isomorphism $\Gamma_{n}(3) \backslash \mathbf{G L}_{n}(\mathbb{Z}) \cong \mathbf{G L}_{n}\left(\mathbb{F}_{3}\right)$. Thus ८ is a monoidal equivalence of categories. In fact, more is true when $p=3$. We claim that the map $\iota^{*}: \operatorname{Mod}_{\mathrm{VB}_{\mathbb{F}_{3}}} \rightarrow \operatorname{Mod}_{\mathrm{VB}_{\mathbb{Z}} / \Gamma(3)}$ induced by $\iota$ is a monoidal equivalence. Note that $\iota$ induces the following isomorphism of transformations

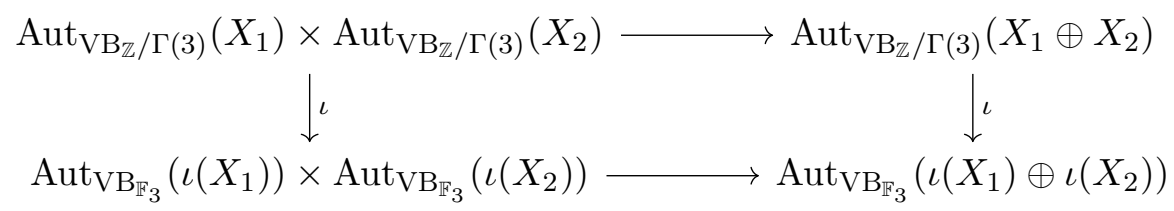

which can easily be verified. Moreover, we have a natural isomorphism (which is equivalent to the strongness of $\Gamma(3)$, and can be easily seen via an application of the second isomorphism theorem)

$$
\iota: \Gamma(3)(X) \backslash \mathbf{G L}(X) /\left(\mathbf{G L}\left(X_{1}\right) \times \mathbf{G L}\left(X_{2}\right)\right) \rightarrow \mathbf{G L}(\iota(X)) /\left(\mathbf{G L}\left(\iota\left(X_{1}\right)\right) \times \mathbf{G L}\left(\iota\left(X_{2}\right)\right)\right)
$$

where $X=X_{1} \oplus X_{2}$. This shows that the left Kan extensions as in Remark 2.1 agrees for the two categories. In particular, $\iota^{*}: \operatorname{Mod}_{\mathrm{VB}_{\mathbb{F}_{3}}} \rightarrow \operatorname{Mod}_{\mathrm{VB}_{\mathbb{Z}} / \Gamma(3)}$ is a monoidal equivalence.

For an odd prime $p$, the category $\mathrm{VB}_{\mathbb{Z}} / \Gamma(p)$ is equivalent to the category $\mathrm{VB}_{\mathbb{F}_{p}}^{ \pm}$which has appeared in the work of Putman-Sam on congruence subgroups [PS17]. An orientation on a finite dimensional $\mathbb{F}_{p}$-vector space $X$ of dimension $n$ is a choice of generator of $\wedge^{n} X$ defined up to multiplication by \pm 1 (we warn the reader that this is different from the usual definition of orientation which is just an isomorphism $\left.\mathbb{F}_{p} \rightarrow \Lambda^{n} X\right)$. Let $\mathrm{VB}_{\mathbb{F}_{p}}^{ \pm}$be the category with objects given by finite dimensional oriented $\mathbb{F}_{p}$-vector spaces and with morphisms given by isomorphisms preserving the orientation. The automorphism groups in this category are isomorphic to $\mathbf{G L}_{n}^{ \pm}\left(\mathbb{F}_{p}\right)$, the subgroup of $\mathbf{G L}_{n}\left(\mathbb{F}_{p}\right)$ consisting of matrices with determinant \pm 1 . Using strongness (Definition 5.3), as in the case $p=3$, one can check that $\operatorname{Mod}_{\mathrm{VB}_{\mathbb{Z}} / \Gamma(p)}$ is monoidally equivalent to $\operatorname{Mod}_{\mathrm{VB}_{\mathbb{F}_{p}}^{ \pm}}$.

From now on, we will assume that $\mathcal{G}$ is an arbitrary strong sub-functor of GL. The reader is advised to keep in mind the sub-functors in the example above as we will focus only on these later.

Proposition 5.5. We have the following:

(a) The pullback functor $\Pi_{\mathcal{G}}^{*}: \operatorname{Mod}_{\mathrm{VB}_{\mathbb{Z}} / \mathcal{G}} \rightarrow \operatorname{Mod}_{\mathrm{VB}_{\mathbb{Z}}}$ has a left adjoint $\mathrm{H}_{0}(\mathcal{G} ;-)$ given by

$$
\mathrm{H}_{0}(\mathcal{G} ; M)(X)=\mathrm{H}_{0}(\mathcal{G}(X) ; M(X)) .
$$

(b) $\mathrm{H}_{0}(\mathcal{G} ;-)$ is strong monoidal. 
Proof. We note that our tensor product is the same as Djament's; see Remark 2.1. Part (a) is proven in [Dja, Proposition 1.3, 1.4]. Now we prove Part (b). In [Dja, §1.5], it is shown that $\mathrm{H}_{0}(\mathcal{G} ;-)$ is lax monoidal. We claim $\mathrm{H}_{0}(\mathcal{G} ;-)$ is in fact strong monoidal which we verify as follows:

$$
\begin{aligned}
\mathrm{H}_{0}(\mathcal{G} ; M \otimes N)(X) & =\mathrm{H}_{0}(\mathcal{G}(X) ;(M \otimes N)(X)) \\
& =\mathrm{H}_{0}\left(\mathcal{G}(X) ; \bigoplus_{X_{1} \oplus X_{2}=X \text { in } \mathrm{VB}_{\mathbb{Z}}} M\left(X_{1}\right) \otimes_{\mathbf{k}} N\left(X_{2}\right)\right) \\
& =\mathrm{H}_{0}\left(\mathcal{G}(X) ; \bigoplus_{X_{1} \oplus X_{2}=X \text { in } \mathrm{VB}_{\mathbb{Z}} / \mathcal{G}} \operatorname{Ind}_{\mathcal{G}(X) \cap\left(\mathbf{G L}\left(X_{1}\right) \times \mathbf{G L}\left(X_{2}\right)\right)}^{\mathcal{G}(X)} M\left(X_{1}\right) \otimes_{\mathbf{k}} N\left(X_{2}\right)\right) \\
& =\bigoplus_{X_{1} \oplus X_{2}=X \text { in } \mathrm{VB}_{\mathbb{Z}} / \mathcal{G}} \mathrm{H}_{0}\left(\mathcal{G}(X) ; \operatorname{Ind}_{\mathcal{G}\left(X_{1}\right) \times \mathcal{G}\left(X_{2}\right)}^{\mathcal{G}(X)} M\left(X_{1}\right) \otimes_{\mathbf{k}} N\left(X_{2}\right)\right) \\
& =\bigoplus_{X_{1} \oplus X_{2}=X \text { in } \mathrm{VB}_{\mathbb{Z}} / \mathcal{G}} \mathrm{H}_{0}\left(\mathcal{G}\left(X_{1}\right) \times \mathcal{G}\left(X_{2}\right) ; M\left(X_{1}\right) \otimes_{\mathbf{k}} N\left(X_{2}\right)\right) \\
& =\left(\mathrm{H}_{0}(\mathcal{G} ; M) \otimes \mathrm{H}_{0}(\mathcal{G} ; N)\right)(X) .
\end{aligned}
$$

where the third equality follows from the Mackey decomposition theorem, the fourth equality follows from the fact that $\mathcal{G}$ is strong, and the last equality follows from the Künneth formula for group homology. This completes the proof.

Since $\mathrm{H}_{0}(\mathcal{G} ;-)$ is monoidal, it takes a monoid $A$ in $\operatorname{Mod}_{\mathrm{VB}_{\mathbb{Z}}}$ to a monoid $B:=\mathrm{H}_{0}(\mathcal{G} ; A)$ in $\operatorname{Mod}_{\mathrm{VB}_{\mathbb{Z}} / \mathcal{G}}$. Moreover, $\mathrm{H}_{0}(\mathcal{G} ;-)$ restricts to a functor

$$
\mathrm{H}_{0}^{A}(\mathcal{G} ;-): \operatorname{Mod}_{A} \rightarrow \operatorname{Mod}_{B} .
$$

The functors $\mathrm{H}_{0}(\mathcal{G} ;-)$ and $\mathrm{H}_{0}^{A}(\mathcal{G} ;-)$ are right exact. We denote their left derived functors by $\mathrm{H}_{*}(\mathcal{G} ;-)$ and $\mathrm{H}_{*}^{A}(\mathcal{G} ;-)$ respectively.

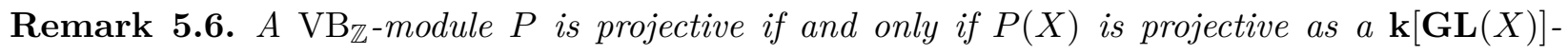
module for all $X$. This shows that $\mathrm{H}_{i}(\mathcal{G} ; M)(X) \cong \mathrm{H}_{i}(\mathcal{G}(X) ; M(X))$. We warn the readers that, for a general monoid $A$, we do not have any natural relationship between $\mathrm{H}_{i}^{A}(\mathcal{G} ; M)(X)$ and $\mathrm{H}_{i}(\mathcal{G}(X) ; M(X))$ for $i>0$. This is because a projective module in $\operatorname{Mod}_{A}$ may not be projective (or even flat) as a $\mathrm{VB}_{\mathbb{Z}}$-module. See Lemma 5.10 for more on this.

Proposition 5.7. $\mathrm{H}_{0}(\Gamma(p) ;-)$ commutes with tensor, symmetric and exterior algebras. In other words, if $M$ is a $\mathrm{VB}_{\mathbb{Z}}$-module, then we have

(a) $\mathrm{H}_{0}(\Gamma(p) ; \mathrm{T}(M))=\mathrm{T}\left(\mathrm{H}_{0}(\Gamma(p) ; M)\right)$ where $\mathrm{T}$ is the tensor algebra.

(b) $\mathrm{H}_{0}(\Gamma(p) ; \operatorname{Sym}(M))=\operatorname{Sym}\left(\mathrm{H}_{0}(\Gamma(p) ; M)\right)$.

(c) $\mathrm{H}_{0}(\Gamma(p) ; \bigwedge(M))=\bigwedge\left(\mathrm{H}_{0}(\Gamma(p) ; M)\right)$.

Proof. Part (a) follows immediately from the fact that $\mathrm{H}_{0}(\mathcal{G} ;-)$ is strong monoidal. Moreover, since the action of the symmetric group on tensors commutes with the action of the congruence subgroup, we see that parts (b) and (c) hold as well.

Proposition 5.8. Let $A$ be an augmented monoid in $\operatorname{Mod}_{\mathrm{VB}_{\mathbb{Z}}}$. Set $B=\mathrm{H}_{0}(\mathcal{G} ; A)$. Then the following diagram commutes. 


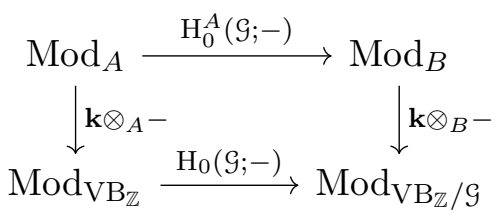

Proof. Let $I_{A}$ and $I_{B}$ be the right adjoints of $\mathbf{k} \otimes_{A}-$ and $\mathbf{k} \otimes_{B}-$ respectively. By our assumption on $A, I_{A}(M)$ is the same as $M$ regarded as an $A$-module via the augmentation map $A \rightarrow \mathbf{k}$. A similar statement is true for $I_{B}$. This shows that $\mathrm{H}_{0}^{A}(\mathcal{G} ;-) \circ I_{A}=I_{B} \circ \mathrm{H}_{0}(\mathcal{G} ;-)$.

Let $\epsilon$ : id $\rightarrow I_{A} \circ\left(\mathbf{k} \otimes_{A}-\right)$ be the unit of the adjunction. By the previous paragraph, we have a natural transformation $\mathrm{H}_{0}^{A}(\mathcal{G} ;-) \rightarrow I_{B} \circ \mathrm{H}_{0}(\mathcal{G} ;-) \circ\left(\mathbf{k} \otimes_{A}-\right)$ obtained by composing $\mathrm{H}_{0}^{A}(\mathcal{G} ;-)$ with the unit $\epsilon$. By adjunction, there exists a natural transformation

$$
\left(\mathbf{k} \otimes_{B}-\right) \circ \mathrm{H}_{0}^{A}(\mathcal{G} ;-) \rightarrow \mathrm{H}_{0}(\mathcal{G} ;-) \circ\left(\mathbf{k} \otimes_{A}-\right)
$$

which we claim is an isomorphism. Since all the functors involved are right exact, it suffices to prove the claim for objects of the form $A \otimes V$ (projective objects are of this form). We now check this as follows:

$$
\begin{aligned}
\left(\mathbf{k} \otimes_{B}-\right) \circ \mathrm{H}_{0}^{A}(\mathcal{G} ;-)(A \otimes V) & =\mathbf{k} \otimes_{B} \mathrm{H}_{0}^{A}(\mathcal{G} ; A \otimes V) \\
& =\mathbf{k} \otimes_{B}\left(\mathrm{H}_{0}(\mathcal{G} ; A) \otimes \mathrm{H}_{0}(\mathcal{G} ; V)\right) \quad \text { since } \mathrm{H}_{0}(\mathcal{G} ;-) \text { is monoidal } \\
& =\mathbf{k} \otimes_{B}\left(B \otimes \mathrm{H}_{0}(\mathcal{G} ; V)\right) \\
& =\mathrm{H}_{0}(\mathcal{G} ; V)=\mathrm{H}_{0}\left(\mathcal{G} ; \mathbf{k} \otimes_{A}(A \otimes V)\right) \\
& =\mathrm{H}_{0}(\mathcal{G} ;-) \circ\left(\mathbf{k} \otimes_{A}-\right)(A \otimes V) .
\end{aligned}
$$

This completes the proof.

Note that $\operatorname{Mod}_{\mathrm{VB}_{\mathbb{Z}}}$ has enough projectives $-P$ is a projective $\mathrm{VB}_{\mathbb{Z}}$-module if and only if $P\left(\mathbb{Z}^{n}\right)$ is a projective $\mathbb{Z}\left[\mathbf{G L}_{n}(\mathbb{Z})\right]$-module for each $n \geq 0$. In particular, we can define left-derived functors $\mathrm{L}_{i} F$ of a given right exact functor $F$ defined on $\operatorname{Mod}_{\mathrm{VB}_{\mathbb{Z}}}$.

Theorem 5.9. Let $A$ be an augmented monoid in $\operatorname{Mod}_{\mathrm{VB}_{\mathbb{Z}}}$. Set $B=\mathrm{H}_{0}(\mathcal{G} ; A)$. Then there exists a spectral sequence

$$
E_{a b}^{2}(-)=\operatorname{Tor}_{a}^{B}\left(\mathbf{k}, \mathrm{H}_{b}^{A}(\mathcal{G} ;-)\right) \Longrightarrow \mathrm{L}_{a+b}\left(\mathrm{H}_{0}(\mathcal{G} ;-) \circ\left(\mathbf{k} \otimes_{A}-\right)\right)
$$

In particular, if $M$ is an $A$-module such that $\max _{i \leq n}\left(\operatorname{deg} \operatorname{Tor}_{i}^{A}(\mathbf{k}, M)\right) \leq d$, then $E_{a b}^{\infty}(M)$ is supported in degrees $\leq d$ for all $a+b \leq n$.

Proof. We first verify that $\mathrm{H}_{0}^{A}(\mathcal{G} ;-)$ preserves projectives. It is an easy fact that every projective $A$-module is of the form $A \otimes V$ where $V$ is a projective VB-module, and the same holds for $B$ modules; see [Nag19, Proposition 3.2] for a proof in a similar setting. Since $\mathrm{H}_{0}(\mathcal{G} ;-)$ is monoidal, we see that $\mathrm{H}_{0}^{A}(\mathcal{G} ; A \otimes V)=B \otimes \mathrm{H}_{0}(\mathcal{G} ; V)$. By definition, $\mathrm{H}_{0}(\mathcal{G} ;-)$ is a left adjoint of an exact functor $\Pi_{\mathcal{G}}^{*}$ and so it preserves projectives. Since $\mathrm{H}_{0}^{A}(\mathcal{G} ; A \otimes V)=B \otimes \mathrm{H}_{0}(\mathcal{G} ; V)$, we conclude that $\mathrm{H}_{0}^{A}(\mathcal{G} ;-)$ preserves projectives. This verifies our claim. 
Note that $\operatorname{Mod}_{B}$ has enough projectives. We conclude that there exists a Grothendieck spectral sequence

$$
E_{a b}^{2}(-)=\operatorname{Tor}_{a}^{B}\left(\mathbf{k}, \mathrm{H}_{b}^{A}(\mathcal{G} ;-)\right) \Longrightarrow \mathrm{L}_{a+b}\left(\left(\mathbf{k} \otimes_{B}-\right) \circ \mathrm{H}_{0}^{A}(\mathcal{G} ;-)\right)
$$

By the previous proposition $\left(\mathbf{k} \otimes_{B}-\right) \circ \mathrm{H}_{0}^{A}(\mathcal{G} ;-)=\mathrm{H}_{0}(\mathcal{G} ;-) \circ\left(\mathbf{k} \otimes_{A}-\right)$, and so the first assertion follows.

It is clear from the last paragraph that $\mathbf{k} \otimes_{A}-$ preserves projectives. Also, ModvB has enough projectives. This shows that we have another Grothendieck spectral sequence

$$
\mathrm{H}_{a}\left(\mathcal{G} ; \operatorname{Tor}_{b}^{A}(\mathbf{k},-)\right) \Longrightarrow \mathrm{L}_{a+b}\left(\mathrm{H}_{0}(\mathcal{G} ;-) \circ\left(\mathbf{k} \otimes_{A}-\right)\right)
$$

If $\max _{i \leq n}\left(\operatorname{deg} \operatorname{Tor}_{i}^{A}(\mathbf{k}, M)\right) \leq d$, then we have $\operatorname{deg} \mathrm{L}_{a+b}\left(\mathrm{H}_{0}(\mathcal{G} ;-) \circ\left(\mathbf{k} \otimes_{A}-\right)\right) \leq d$ for all $a+b \leq n$ (also see Remark 5.6). This proves the second assertion.

The following lemma shows that our spectral sequence is particularly useful when $A$ is the exterior algebra (see Remark 5.6). Similar results hold for tensor and symmetric algebras.

Lemma 5.10. Assume that $\mathcal{G}(X)$ is torsion-free for all $X$ (this happens, for example, when $p$ is an odd prime and $\mathcal{G}=\Gamma(p))$. Let $\mathbf{A}=\bigwedge\left(\operatorname{triv}_{1}\right)$ be the apartment monoid in $\mathrm{Mod}_{\mathrm{VB}_{\mathbb{Z}}}$. Then for every $\mathbf{A}$-module $M$ and every $X \in \mathrm{VB}_{\mathbb{Z}}$, we have an isomorphism

$$
\mathrm{H}_{i}^{\mathbf{A}}(\mathcal{G} ; M)(X) \cong \mathrm{H}_{i}(\mathcal{G}(X) ; M(X))
$$

of $\mathbf{G L}(X)$-modules for each $i \geq 0$.

Proof. Note that $\mathrm{H}_{i}^{\mathbf{A}}(\mathcal{G} ; M)$ is calculated by first taking an A-module projective resolution $P_{*} \rightarrow$ $M \rightarrow 0$ of $M$, applying $\mathcal{G}$-coinvariants to the resolution, and then taking $i$ th homology of the resulting complex $\left(P_{*}\right)_{\mathcal{G}}$. Thus to prove the isomorphism in the assertion, it suffices to show that a projective A-module is $\mathrm{H}_{0}\left(\mathcal{G}\right.$; - )-acyclic when regarded as a $\mathrm{VB}_{\mathbb{Z}}$-module (recall that $\mathrm{Mod}_{\mathrm{VB}_{\mathbb{Z}}}$ is equivalent to the product category $\prod_{\geq 0} \operatorname{Mod}_{\mathbf{k}\left[\mathbf{G L}_{n}(\mathbb{Z})\right]}$, and so a module is projective if and only if it is pointwise projective). Let $P$ be a projective $\mathbf{A}$-module. Then $P$ is of the form $\mathbf{A} \otimes V$ for some projective $\mathrm{VB}_{\mathbb{Z}}$-module $V$. We claim that $\mathrm{H}_{i}(\mathcal{G}(X) ; P(X))=0$ for all $X$ and all $i>0$. Note that we have:

$$
\begin{aligned}
\mathrm{H}_{i}(\mathcal{G}(X) ; P(X)) & =\mathrm{H}_{i}(\mathcal{G} ; \mathbf{A} \otimes V)(X) \\
& =\left(\mathrm{H}_{i}(\mathcal{G} ; \mathbf{A}) \otimes \mathrm{H}_{0}(\mathcal{G} ; V)\right)(X)
\end{aligned}
$$

where the first equality follows from Remark 5.6. The second equality is obtained by taking a projective resolution $Q_{*} \rightarrow \mathbf{A}$ in $\operatorname{Mod}_{\mathrm{VB}_{\mathbb{Z}}}$, and then calculating $\mathrm{H}_{i}(\mathcal{G} ; \mathbf{A} \otimes V)$ using the projective resolution $Q_{*} \otimes V \rightarrow \mathbf{A} \otimes V$ and monoidality of $\mathrm{H}_{0}(\mathcal{G} ;-)$. Thus, it suffices to show that $\mathrm{H}_{i}(\mathcal{G} ; \mathbf{A})=0$ for $i>0$. We have that $\mathbf{A}\left(\mathbb{Z}^{n}\right)$ is isomorphic to $\mathrm{H}_{0}\left(\mathbb{Z} / 2 \imath S_{n} ; \mathbf{k}\left[\mathbf{G L}_{n}(\mathbb{Z})\right]\right)$ where $\mathbb{Z} / 2 \imath S_{n}$ acts on the right as in the definition of the apartment monoid. Since $\mathbb{Z} / 2 \imath S_{n}$ is a finite group, the stabilizer of $\mathcal{G}\left(\mathbb{Z}^{n}\right)$ acting on $\mathbf{A}\left(\mathbb{Z}^{n}\right)$ must have finite order. Since $\mathcal{G}\left(\mathbb{Z}^{n}\right)$ is torsion free, this stabilizer is trivial. Thus, $\mathbf{A}(X)$ is a free $\mathbf{k}[\mathcal{G}(X)]$-module. This shows that $\mathrm{H}_{i}(\mathcal{G} ; \mathbf{A})(X)=0$ for $i>0$. This verifies our claim, and the proof is complete. 
Often a $\mathrm{H}_{0}(\mathcal{G} ; A)$ structure on $\mathrm{H}_{i}(\mathcal{G} ; A)$ is defined directly using the Künneth map and the Shapiro lemma instead of relying on any isomorphisms as in the previous lemma. We now describe it and compare it with our setup. For this, we define an enrichment

$$
\mathrm{H}_{*}(\mathcal{G} ;-): \operatorname{Mod}_{\mathrm{VB}_{\mathbb{Z}}} \rightarrow \operatorname{Mod}_{\mathrm{VB}_{\mathbb{Z}} / \mathcal{G}}^{\mathrm{gr}}
$$

of $\mathrm{H}_{0}(\mathcal{G} ;-)$, where $\mathrm{Mod}_{\mathrm{VB}_{\mathbb{Z}} / \mathcal{G}}^{\mathrm{gr}}$ is the category of graded $\mathrm{VB}_{\mathbb{Z}} / \mathcal{G}$-modules, by

$$
\mathrm{H}_{*}(\mathcal{G} ;-)=\bigoplus_{t \geq 0} \mathrm{H}_{t}(\mathcal{G} ;-)
$$

Note $\mathrm{H}_{*}(\mathcal{G} ; M)$ is bigraded with one grading coming from the $\mathrm{VB}_{\mathbb{Z}} / \mathcal{G}$-module structure and the other grading coming from homological degree. The tensor product on $\operatorname{Mod}_{\mathrm{VB}_{\mathbb{Z}} / \mathcal{G}}^{\mathrm{gr}}$ is the usual convolutional tensor product with respect to both of the gradings.

Proposition 5.11. $\mathrm{H}_{*}(\mathcal{G} ;-)$ is a lax monoidal functor.

Proof. The natural map $\mathrm{H}_{*}(\mathcal{G} ; M) \otimes \mathrm{H}_{*}(\mathcal{G} ; N) \rightarrow \mathrm{H}_{*}(\mathcal{G} ; M \otimes N)$ given by composing the Künneth map with the Shapiro isomorphism provides the required lax monoidal structure. The details are very similar to the ones in Proposition 5.5 Part (b). We get lax monoidality instead of strong monoidality because the Künneth map is not an isomorphism in general.

The proposition above shows that if $A$ is a monoid and $M$ is an $A$-module, then $\mathrm{H}_{*}(\mathcal{G} ; A)$ is a monoid and $\mathrm{H}_{*}(\mathcal{G} ; M)$ is an $\mathrm{H}_{*}(\mathcal{G} ; A)$-module. Note that $\mathrm{H}_{0}(\mathcal{G} ; A)$ is naturally a sub-monoid of $\mathrm{H}_{*}(\mathcal{G} ; A)$, and so $\mathrm{H}_{*}(\mathcal{G} ; M)$ is an $\mathrm{H}_{0}(\mathcal{G} ; A)$-module. Let

$$
\mathrm{H}_{*}^{A}(\mathcal{G} ; M)=\bigoplus_{t \geq 0} \mathrm{H}_{t}^{A}(\mathcal{G} ; M) .
$$

By definition, $\mathrm{H}_{*}^{A}(\mathcal{G} ; M)$ is an $\mathrm{H}_{0}(\mathcal{G} ; A)$-module. We now relate the $\mathrm{H}_{0}(\mathcal{G} ; A)$-modules $\mathrm{H}_{*}(\mathcal{G} ; M)$ and $\mathrm{H}_{*}^{A}(\mathcal{G} ; M)$.

Proposition 5.12. Suppose $f: A \rightarrow B$ is a map of monoids in $\operatorname{Mod}_{\mathrm{VB}_{\mathbb{Z}}}$ and assume that projective A-modules are $\mathrm{H}_{*}(\mathcal{G} ;-)$-acyclic. Then we have the following:

(a) For any $A$-module $M, \mathrm{H}_{*}^{A}(\mathcal{G} ; M)$ and $\mathrm{H}_{*}(\mathcal{G} ; M)$ are isomorphic as $\mathrm{H}_{0}(\mathcal{G} ; A)$-modules.

(b) Denote the isomorphism in the previous part by $\varphi$. For any $B$-module $M$, the following diagram commutes:

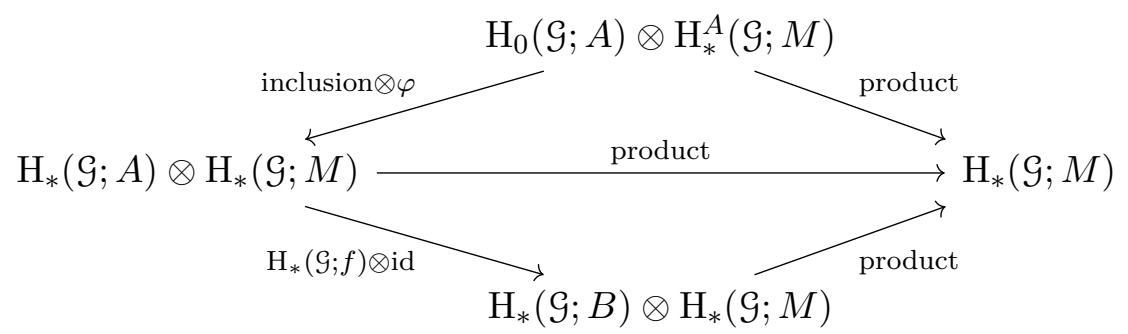

Proof. Proof of Part (a). It is clear from the $\mathrm{H}_{*}(\mathcal{G} ;-)$-acyclicity of projective $A$-modules that $\mathrm{H}_{*}^{A}(\mathcal{G} ; M)$ and $\mathrm{H}_{*}(\mathcal{G} ; M)$ are isomorphic as $\operatorname{Mod}_{\mathrm{VB}_{\mathbb{Z}} / \mathcal{G}^{-m o d u l e s . ~ I n ~ p a r t i c u l a r, ~ a n ~} A \text {-projective }}^{\mathrm{gr}}$ resolution $P_{*} \rightarrow M$ of $M$ can be used to calculate both $\mathrm{H}_{*}(\mathcal{G} ; M)$ and $\mathrm{H}_{*}^{A}(\mathcal{G} ; M)$. The $\mathrm{H}_{0}(\mathcal{G} ; A)$ 
action on $\mathrm{H}_{0}\left(\mathcal{G} ; P_{i}\right)$ is given by composing the Künneth map and the Shapiro isomorphism. Since these two maps are functorial, we see that the action of $\mathrm{H}_{0}(\mathcal{G} ; A)$ on

$$
\mathrm{H}_{i}\left(\mathrm{H}_{0}\left(\mathcal{G} ; P_{*}\right)\right)=\mathrm{H}_{i}^{A}(\mathcal{G} ; M)
$$

is given by composing the Künneth map and the Shapiro isomorphism. This completes the proof of Part (a).

We now prove Part (b). The commutativity of the top triangle is exactly Part (a). The bottom triangle commutes because of the proposition above and functoriality of the Künneth and the Shapiro maps. This finishes the proof.

5.2. Proof of Theorem 5.1 (the main theorem on congruence subgroup). We now use our main technical result (Theorem 4.2) on the Steinberg monoid as an apartment module and the spectral sequence in Theorem 5.9 to prove Theorem 5.1 on level 3 congruence subgroup. We first explain how to use our spectral sequence.

Let $\mathbf{A}$ and $\mathbf{S t}$ be the apartment and the Steinberg monoids in $\operatorname{Mod}_{\mathrm{VB}_{\mathbb{Z}}}$. Let $p$ be a prime, and let $\Gamma(p)$ be as described in (2). Applying Theorem 5.9 to the A-module $\mathbf{S t}$, we obtain the following spectral sequence:

$$
E_{a b}^{2}=\operatorname{Tor}_{a}^{\mathrm{H}_{0}(\Gamma(p) ; \mathbf{A})}\left(\mathbf{k}, \mathrm{H}_{b}^{\mathbf{A}}(\Gamma(p) ; \mathbf{S t})\right) \Longrightarrow \mathrm{L}_{a+b}\left(\mathrm{H}_{0}(\Gamma(p) ;-) \circ\left(\mathbf{k} \otimes_{\mathbf{A}}-\right)\right)(\mathbf{S t}) .
$$

We now simplify this spectral sequence. Since $\mathrm{H}_{0}(\Gamma(p) ;-)$ is monoidal, $\mathrm{H}_{0}(\Gamma(p) ; \mathbf{A})$ is a monoid in $\operatorname{Mod}_{\mathrm{VB}_{\mathbb{Z}} / \Gamma(p)}$ and $\mathrm{H}_{0}(\Gamma(p) ; \mathbf{S t})$ is a module over it. For brevity, we set $\mathbf{A}_{\Gamma(p)}:=\mathrm{H}_{0}(\Gamma(p) ; \mathbf{A})$ and $\mathbf{S t}_{\Gamma(p)}:=\mathrm{H}_{0}(\Gamma(p) ; \mathbf{S t})$. Lemma 5.10 tells us that $\mathrm{H}_{b}^{\mathbf{A}}(\Gamma(p) ;-)$ is isomorphic to $\mathrm{H}_{b}(\Gamma(p) ;-)$ as $\mathrm{VB}_{\mathbb{Z}} / \Gamma(p)$-modules, but comes equipped with an action of $\mathbf{A}_{\Gamma(p)}$. Thus we can drop the superscript A without causing any issues. We now obtain the following simplified spectral sequence

$$
E_{a b}^{2}=\operatorname{Tor}_{a}^{\mathbf{A}_{\Gamma(p)}}\left(\mathbf{k}, \mathrm{H}_{b}(\Gamma(p) ; \mathbf{S t})\right) \Longrightarrow \mathrm{L}_{a+b}\left(\mathrm{H}_{0}(\Gamma(p) ;-) \circ\left(\mathbf{k} \otimes_{\mathbf{A}}-\right)\right)(\mathbf{S t}) .
$$

We need one more ingredient to be able to use this spectral sequence to prove our main theorem. The following proposition is this ingredient and is precisely the place where we use our main technical result (Theorem 4.2) and the assumption that $p=3$. We defer the proof of this proposition until the next subsection and concentrate on using it first.

Proposition 5.13. We have $\operatorname{deg} \operatorname{Tor}_{i}^{\mathbf{A}_{\Gamma(3)}}\left(\mathbf{k}, \mathbf{S t}_{\Gamma(3)}\right) \leq 2 i$ for all $i \geq 0$. Moreover, we have $\operatorname{dim}_{\mathbf{k}} \mathbf{S t}_{\Gamma(3)}\left(\mathbb{Z}^{n}\right)=3^{\left(\begin{array}{c}n \\ 2\end{array}\right)}$.

The following proposition is a more abstract version of our main theorem.

Proposition 5.14. $\mathrm{H}_{1}(\Gamma(3) ; \mathbf{S t})$ is an $\mathbf{A}_{\Gamma(3)}$-module generated in degrees $\leq 4$.

Proof. When $p=3$, the spectral sequence (3) becomes

$$
E_{a b}^{2}=\operatorname{Tor}_{a}^{\mathbf{A}_{\Gamma(3)}}\left(\mathbf{k}, \mathrm{H}_{b}(\Gamma(3) ; \mathbf{S t})\right) \Longrightarrow \mathrm{L}_{a+b}\left(\mathrm{H}_{0}(\Gamma(3) ;-) \circ\left(\mathbf{k} \otimes_{\mathbf{A}}-\right)\right)(\mathbf{S t}) .
$$

Note that $\operatorname{deg} \operatorname{Tor}_{i}^{\mathbf{A}}(\mathbf{k}, \mathbf{S t}) \leq 2 i$ for $i=0,1$ (by Proposition 4.1). Thus by the second assertion of Theorem 5.9, we see that $E_{a b}^{\infty}$ is supported in degrees $\leq 2$ for $a+b \leq 1$. Now note that $E_{0,1}^{\infty}$ is the cokernel of the map

$$
\operatorname{Tor}_{2}^{\mathbf{A}_{\Gamma(3)}}\left(\mathbf{k}, \mathrm{H}_{0}(\Gamma(3) ; \mathbf{S t})\right)=\operatorname{Tor}_{2}^{\mathbf{A}_{\Gamma(3)}}\left(\mathbf{k}, \mathbf{S t}_{\Gamma(3)}\right) \rightarrow \operatorname{Tor}_{0}^{\mathbf{A}_{\Gamma(3)}}\left(\mathbf{k}, \mathrm{H}_{1}(\Gamma(3) ; \mathbf{S t})\right) .
$$


By the previous proposition, $\operatorname{Tor}_{2}^{\mathbf{A}_{\Gamma(3)}}\left(\mathbf{k}, \mathbf{S t}_{\Gamma(3)}\right)$ is supported in degrees $\leq 4$, and so we conclude that

$$
\operatorname{deg} \operatorname{Tor}_{0}^{\mathbf{A}_{\Gamma(3)}}\left(\mathbf{k}, \mathrm{H}_{1}(\Gamma(3) ; \mathbf{S t})\right) \leq 4,
$$

completing the proof of the proposition.

Corollary 5.15. $\mathrm{H}_{1}(\Gamma(3) ; \mathbf{S t})$ is a $\mathbf{S t}_{\Gamma(3)}$-module generated in degrees $\leq 4$.

Proof. This is immediate from the proposition above and Proposition 5.12 Part (b).

Remark 5.16. Bounds on $\operatorname{deg} \operatorname{Tor}_{2}^{\mathbf{A}}(\mathbf{k}, \mathbf{S t})$ could be used together with the arguments of this paper to bound $\operatorname{Tor}_{1}^{\mathbf{A}_{\Gamma(3)}}\left(\mathbf{k}, \mathrm{H}_{1}(\Gamma(3) ; \mathbf{S t})\right)$. Similarly, bounds on $\operatorname{deg} \operatorname{Tor}_{i}^{\mathbf{A}}(\mathbf{k}, \mathbf{S t})$ seem likely to be useful for bounding degrees of the higher syzygies $\operatorname{Tor}_{i}^{\mathbf{A}_{\Gamma(3)}}\left(\mathbf{k}, \mathrm{H}_{j}(\Gamma(3) ; \mathbf{S t})\right)$. However, bounds on $\operatorname{deg} \operatorname{Tor}_{i}^{\mathbf{A}}(\mathbf{k}, \mathbf{S t})$ are not known for $i>1$.

Proof of Theorem 5.1. Proposition 5.14 implies that

$$
\bigoplus_{\substack{X_{1} \oplus X_{2}=X \text { in } \mathrm{VB}_{\mathbb{Z}} / \Gamma(3), \operatorname{rank} X_{2}=4}} \mathbf{A}_{\Gamma(3)}\left(X_{1}\right) \otimes_{\mathbf{k}} \mathrm{H}_{1}(\Gamma(3) ; \mathbf{S t})\left(X_{2}\right) \rightarrow \mathrm{H}_{1}(\Gamma(3) ; \mathbf{S t})(X)
$$

is surjective for $\operatorname{rank} X \geq 4$. Setting $X=\mathbb{Z}^{n}$ for $n \geq 4$, we obtain the following surjection:

$$
\operatorname{Ind}_{\mathrm{Aut}_{\mathrm{VB}_{\mathbb{Z}} / \Gamma(3)}\left(\mathbb{Z}^{n-4}\right) \times \operatorname{Aut}_{\mathrm{VB}_{\mathbb{Z}} / \Gamma(3)}\left(\mathbb{Z}^{4}\right)}^{\mathrm{Aut}_{\mathrm{B}_{\mathrm{Z}} / \Gamma(3)}\left(\mathbb{Z}^{n}\right)} \mathbf{A}_{\Gamma(3)}\left(\mathbb{Z}^{n-4}\right) \otimes_{\mathbf{k}} \mathrm{H}_{1}(\Gamma(3) ; \mathbf{S t})\left(\mathbb{Z}^{4}\right) \rightarrow \mathrm{H}_{1}(\Gamma(3) ; \mathbf{S t})\left(\mathbb{Z}^{n}\right) .
$$

As noted in Example 5.4, we have $\operatorname{Aut}_{\mathrm{VB}_{\mathbb{Z}} / \Gamma_{3}}\left(\mathbb{Z}^{n}\right)=\mathbf{G L}_{n}\left(\mathbb{F}_{3}\right)$. Using the monoidal equivalence $\iota^{*}$ as in that example, we see that the following map is a surjection:

$$
\operatorname{Ind}_{\mathbf{G L}_{n-4}\left(\mathbb{F}_{3}\right) \times \mathbf{G L}_{4}\left(\mathbb{F}_{3}\right)}^{\mathbf{G L}_{n}\left(\mathbb{F}_{3}\right)} \mathbf{A}_{\Gamma(3)}\left(\mathbb{Z}^{n-4}\right) \otimes_{\mathbf{k}} \mathrm{H}_{1}(\Gamma(3) ; \mathbf{S t})\left(\mathbb{Z}^{4}\right) \rightarrow \mathrm{H}_{1}(\Gamma(3) ; \mathbf{S t})\left(\mathbb{Z}^{n}\right) .
$$

We also have a natural surjection:

$$
\mathbf{k}\left[\operatorname{Aut}_{\mathrm{VB}_{\mathbb{Z}} / \Gamma(3)}\left(\mathbb{Z}^{n-4}\right)\right]=\mathbf{k}\left[\mathbf{G L}_{n-4}\left(\mathbb{F}_{3}\right)\right] \rightarrow \mathbf{A}_{\Gamma(3)}\left(\mathbb{Z}^{n-4}\right) .
$$

Combining it with (4), we obtain the desired surjection:

$$
\operatorname{Ind}_{\mathbf{G L}_{4}\left(\mathbb{F}_{3}\right)}^{\mathbf{G L}_{n}\left(\mathbb{F}_{3}\right)} \mathrm{H}_{1}(\Gamma(3) ; \mathbf{S t})\left(\mathbb{Z}^{4}\right) \rightarrow \mathrm{H}_{1}(\Gamma(3) ; \mathbf{S t})\left(\mathbb{Z}^{n}\right) .
$$

The first assertion now follows from the equality $\mathrm{H}_{1}(\Gamma(3) ; \mathbf{S t})\left(\mathbb{Z}^{n}\right)=\mathrm{H}_{1}\left(\Gamma_{n}(3) ; \mathbf{S t}_{n}(\mathbb{Z})\right)$.

For the second assertion, note that by Corollary 5.15 we have the following analogue of (4):

$$
\operatorname{Ind}_{\mathbf{G L}_{n-4}\left(\mathbb{F}_{3}\right) \times \mathbf{G L}_{4}\left(\mathbb{F}_{3}\right)}^{\mathbf{G L}_{n}\left(\mathbb{F}_{3}\right)} \mathbf{S t}_{\Gamma(3)}\left(\mathbb{Z}^{n-4}\right) \otimes_{\mathbf{k}} \mathrm{H}_{1}(\Gamma(3) ; \mathbf{S t})\left(\mathbb{Z}^{4}\right) \rightarrow \mathrm{H}_{1}(\Gamma(3) ; \mathbf{S t})\left(\mathbb{Z}^{n}\right) .
$$

The second assertion now follows from the fact that $\operatorname{dim}_{\mathbf{k}} \mathbf{S t}_{\Gamma(3)}\left(\mathbb{Z}^{n-4}\right)=3^{\left(\begin{array}{c}n-4 \\ 2\end{array}\right)}$.

We now concentrate on proving the dimension bounds in Corollary 5.2.

Lemma 5.17. Suppose $\mathbf{k}$ is a field. We have $\operatorname{dim}_{\mathbf{k}} \mathrm{H}_{1}\left(\Gamma_{3}(3) ; \operatorname{St}_{3}(\mathbb{Z}) \otimes_{\mathbb{Z}} \mathbf{k}\right) \leq 35$.

Proof. By [LS76b, Theorem 1.4], we have $\operatorname{dim}_{\mathbf{k}} \operatorname{Hom}_{\mathbb{Z}}\left(\mathrm{H}_{2}\left(\Gamma_{3}(3)\right), \mathbf{k}\right) \leq 27$. Moreover, by [LS76b, Lemma 12.1] we have

$$
\operatorname{dim}_{\mathbf{k}} \operatorname{Ext}_{\mathbb{Z}}^{1}\left(\mathrm{H}_{2}\left(\Gamma_{3}(3)\right), \mathbf{k}\right)= \begin{cases}8 & \text { if } \operatorname{Char}(\mathbf{k})=3 \\ 0 & \text { otherwise }\end{cases}
$$


Thus by the universal coefficient theorem, we have $\operatorname{dim}_{\mathbf{k}} \mathrm{H}^{2}\left(\Gamma_{3}(3) ; \mathbf{k}\right) \leq 35$. By Borel-Serre duality, we conclude that $\operatorname{dim}_{\mathbf{k}} \mathrm{H}_{1}\left(\Gamma_{3}(3) ; \operatorname{St}_{3}(\mathbb{Z}) \otimes_{\mathbb{Z}} \mathbf{k}\right) \leq 35$.

The following lemma and Theorem 5.1 complete the proof of Corollary 5.2.

Lemma 5.18. Suppose $\mathbf{k}$ is a field. Then we have

$$
\operatorname{dim}_{\mathbf{k}} \mathrm{H}_{1}\left(\Gamma_{4}(3) ; \operatorname{St}_{4}(\mathbb{Z}) \otimes_{\mathbb{Z}} \mathbf{k}\right)=\operatorname{dim}_{\mathbf{k}} \mathrm{H}_{1}(\Gamma(3) ; \mathbf{S t})\left(\mathbb{Z}^{4}\right) \leq 227340 .
$$

Proof. Denote the $\mathbf{A}_{\Gamma(3)}$-module $\mathrm{H}_{1}(\Gamma(3) ; \mathbf{S t})$ by $M$, and let $M^{\prime}$ be the maximal submodule of $M$ generated in degrees $\leq 3$. Let $V$ be the degree 4 piece of the $\mathrm{VB}_{\mathbb{Z}} / \Gamma(3)$-module $\operatorname{Tor}_{0}^{\mathbf{A}_{\Gamma(3)}}\left(\mathbf{k}, \mathrm{H}_{1}(\Gamma(3) ; \mathbf{S t})\right)$. Then by the definition of $\operatorname{Tor}_{0}^{\mathbf{A}_{\Gamma(3)}}(\mathbf{k},-)$, we have an isomorphism

$$
V\left(\mathbb{Z}^{4}\right) \cong\left(M / M^{\prime}\right)\left(\mathbb{Z}^{4}\right)
$$

This shows that

$$
\operatorname{dim}_{\mathbf{k}} M\left(\mathbb{Z}^{4}\right)=\operatorname{dim}_{\mathbf{k}} M^{\prime}\left(\mathbb{Z}^{4}\right)+\operatorname{dim}_{\mathbf{k}} V\left(\mathbb{Z}^{4}\right)
$$

The proof of Proposition 5.14 shows that the map $\operatorname{Tor}_{2}^{\mathbf{A}_{\Gamma(3)}}\left(\mathbf{k}, \mathbf{S t}_{\Gamma(3)}\right) \rightarrow \operatorname{Tor}_{0}^{\mathbf{A}_{\Gamma(3)}}\left(\mathbf{k}, \mathrm{H}_{1}(\Gamma(3) ; \mathbf{S t})\right)$ is surjective in degrees 3 and 4 . In particular, we have

$$
\operatorname{dim}_{\mathbf{k}} V\left(\mathbb{Z}^{4}\right) \leq \operatorname{dim}_{\mathbf{k}} \operatorname{Tor}_{2}^{\mathbf{A}_{\Gamma(3)}}\left(\mathbf{k}, \mathbf{S t}_{\Gamma(3)}\right)\left(\mathbb{Z}^{4}\right)
$$

By Proposition 5.19 and Lemma 3.9 (for $q=3$ ), we have

$$
\operatorname{dim}_{\mathbf{k}} \operatorname{Tor}_{2}^{\mathbf{A}_{\Gamma(3)}}\left(\mathbf{k}, \mathbf{S t}_{\Gamma(3)}\right)\left(\mathbb{Z}^{4}\right) \leq \frac{\left(3^{4}-1\right)\left(3^{3}-1\right) 3^{6}}{8}=189540 .
$$

By the previous lemma, $\operatorname{dim}_{\mathbf{k}} M^{\prime}\left(\mathbb{Z}^{3}\right) \leq 35$. Since $M^{\prime}$ is generated in degrees $\leq 3$, we see that

$$
\operatorname{dim}_{\mathbf{k}} M^{\prime}\left(\mathbb{Z}^{4}\right) \leq \frac{35\left|\mathbf{G L}_{4}\left(\mathbb{F}^{3}\right)\right|}{\left|\mathbf{G L}_{3}\left(\mathbb{F}^{3}\right) \times \mathbf{G L}_{1}\left(\mathbb{F}^{3}\right)\right|} \leq 37800 .
$$

Finally, we conclude that $\operatorname{dim}_{\mathbf{k}} M\left(\mathbb{Z}^{4}\right) \leq 189540+37800=227340$. This completes the proof.

5.3. Proof of Proposition 5.13. Using the monoidal equivalence $\iota^{*}: \operatorname{Mod}_{\mathrm{VB}_{\mathbb{F}_{3}}} \rightarrow \operatorname{Mod}_{\mathrm{VB}_{\mathbb{Z}} / \Gamma(3)}$ as in Example 5.4, we now identify $\operatorname{Mod}_{\mathrm{VB}_{\mathbb{Z}} / \Gamma(3)}$ with $\operatorname{Mod}_{\mathrm{VB}_{\mathbb{F}_{3}}}$. Proposition 5.13 is immediate

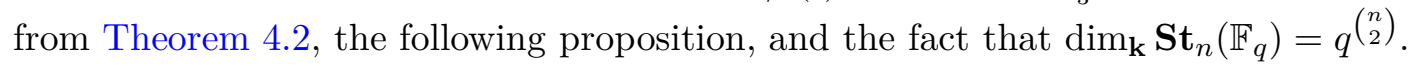

Proposition 5.19. The monoid $\mathbf{A}_{\Gamma(3)}$ is naturally isomorphic to the apartment monoid in $\operatorname{Mod}_{\mathrm{VB}_{\mathbb{F}_{3}}}$, and under this isomorphism, $\mathbf{S t}_{\Gamma(3)}$ is the Steinberg monoid in $\operatorname{Mod}_{\mathrm{VB}_{\mathbb{F}_{3}}}$.

Proof. The first assertion follows immediately from Proposition 5.7 and the fact that the apartment monoid is an exterior algebra.

Let us denote the apartment and the Steinberg monoids in $\operatorname{Mod}_{\mathrm{VB}_{\mathbb{F}_{3}}}$ by $\overline{\mathbf{A}}$ and $\overline{\mathbf{S t}}$ respectively. By Theorem 2.5 and Theorem 2.8 (also see the proof of Proposition 4.1), we have an exact sequence of A-modules

$$
\mathbf{A} \otimes M \rightarrow \mathbf{A} \rightarrow \mathbf{S t} \rightarrow 0
$$

and an exact sequence of $\overline{\mathbf{A}}$-modules

$$
\overline{\mathbf{A}} \otimes \bar{M} \rightarrow \overline{\mathbf{A}} \rightarrow \overline{\mathbf{S t}} \rightarrow 0 .
$$


Here $M$ and $\bar{M}$ are supported only in degree 2 and are given by

$$
M\left(\mathbb{Z}^{2}\right)=\left\langle\left[v_{1}, v_{2}\right]-\left[v_{0}, v_{2}\right]+\left[v_{0}, v_{1}\right] \mid v_{0}=v_{1}+v_{2}\right\rangle \subset \mathbf{A}\left(\mathbb{Z}^{2}\right)
$$

and

$$
\bar{M}\left(\mathbb{F}_{3}^{2}\right)=\left\langle\left[v_{1}, v_{2}\right]-\left[v_{0}, v_{2}\right]+\left[v_{0}, v_{1}\right] \mid v_{0}=v_{1}+v_{2}\right\rangle \subset \overline{\mathbf{A}}\left(\mathbb{F}_{3}^{2}\right) .
$$

We note that the units in $\mathbb{Z}$ and $\mathbb{F}_{3}$ are both $\{1,-1\}$. Applying the monoidal functor $\mathrm{H}_{0}(\Gamma(3) ;-)$ to Equation (6), we obtain an exact sequence:

$$
\mathbf{A}_{\Gamma(3)} \otimes \mathrm{H}_{0}(\Gamma(3) ; M) \rightarrow \mathbf{A}_{\Gamma(3)} \rightarrow \mathbf{S t}_{\Gamma(3)} \rightarrow 0
$$

which we will show is isomorphic to Equation (7). Under the isomorphism $\mathbf{A}_{\Gamma(3)} \rightarrow \overline{\mathbf{A}}$ as in the first assertion, we note that both $\mathbf{S t}_{\Gamma(3)}$ and $\overline{\mathbf{S t}}$ are quotients of $\overline{\mathbf{A}}$ by an ideal generated in degree 2. To show that the two ideals are the same, all we need is to check that the image of $\mathrm{H}_{0}\left(\Gamma_{2}(3) ; M\left(\mathbb{Z}^{2}\right)\right) \rightarrow \mathrm{H}_{0}\left(\Gamma_{2}(3) ; \mathbf{A}\left(\mathbb{Z}^{2}\right)\right)$ is equal to the image of $\bar{M}\left(\mathbb{F}_{3}^{2}\right) \rightarrow \overline{\mathbf{A}}\left(\mathbb{F}_{3}^{2}\right)$ under the isomorphism $\mathrm{H}_{0}\left(\Gamma_{2}(3) ; \mathbf{A}\left(\mathbb{Z}^{2}\right)\right) \rightarrow \overline{\mathbf{A}}\left(\mathbb{F}_{3}^{2}\right)$. This last isomorphism is just reduction $\bmod p$ and is given by

$$
\left[v_{1}, v_{2}\right] \mapsto\left[\bar{v}_{1}, \bar{v}_{2}\right]
$$

The statement about images being equal now follows immediately from the explicit descriptions of $M$ and $\bar{M}$ (see [LS76b, Lemma 5.2] for a similar argument).

Remark 5.20. Lee-Szczarba [LS76b, Theorem 1.2] proved that $\operatorname{St}_{n}(\mathbb{Z})_{\Gamma_{n}(3)} \cong \operatorname{St}_{n}\left(\mathbb{F}_{3}\right)$ for $n \geq 3$. Proposition 5.19 shows that this is in fact true for all $n$.

\section{A stability Conjecture}

In [CFP14], Church, Farb, and Putman conjectured that for all $i$, the codimension $i$ rational cohomology of mapping class groups, automorphism groups of free groups, and $\mathbf{S L}_{n}(\mathbb{Z})$ stabilize. Here codimension $i$ means $i$ below the virtual cohomological dimension. These groups are rational duality groups so this stability conjecture is equivalent to homological stability with coefficients in the dualizing modules tensor $\mathbb{Q}$. In particular, they conjectured that $\mathrm{H}_{i}\left(\mathbf{S L}_{n}(\mathbb{Z}) ; \mathrm{St}_{n}(\mathbb{Z}) \otimes \mathbb{Q}\right)$ does not depend on $n$ for $n \gg i$. In the case of $\mathrm{H}_{i}\left(\mathbf{S L}_{n}(\mathbb{Z}) ; \mathrm{St}_{n}(\mathbb{Z}) \otimes \mathbb{Q}\right)$, if one could show that these groups stabilized, it would also imply that the stable homology groups are zero [CFP14, §2].

Since it is known that the cohomology groups of congruence subgroups in the virtual cohomological dimension are nonzero and in fact grow with $n$ [LS76b, Par97], Church, Farb, and Putman did not conjecture that the cohomology of congruence subgroups vanishes or stabilizes in high dimensions. Nevertheless, they said regarding the codimension $i$ cohomology of congruence subgroups that they "do expect that the stability conjectured in Conjecture 1 should persist in some form" [CFP14, Page 7]. Given that the untwisted homology of congruence subgroups exhibits representation stability, it is reasonable to conjecture that the stability pattern exhibited by the homology of congruence subgroups with coefficients in Steinberg modules should also be a form of representation stability. We propose the following conjecture as a way of making Church-Farb-Putman's statement more precise. 
Conjecture 6.1. Let $p$ be a prime. For each $i, j \geq 0$, the $\mathrm{VB}_{\mathbb{Z}} / \Gamma(p)$-module

$$
\operatorname{Tor}_{i}^{\mathbf{A}_{\Gamma(p)}}\left(\mathbf{k}, \mathrm{H}_{j}(\Gamma(p) ; \mathbf{S t})\right)
$$

is supported in finitely many degrees.

For simplicity, we concentrate on the case when $p$ is an odd prime so that the units of $\mathbb{Z}$ will inject into the units of $\mathbb{F}_{p}$ and so that $\Gamma_{n}(p)$ will be torsion free and hence exhibit integral BorelSerre duality. As explained in Example $5.4, \mathrm{VB}_{\mathbb{Z}} / \Gamma(p)$ is monoidally equivalent to an oriented version $\mathrm{VB}_{\mathbb{F}_{p}}^{ \pm}$of $\mathrm{VB}_{\mathbb{F}_{p}}$. Moreover, it induces a monoidal equivalence between $\operatorname{Mod}_{\mathrm{VB}_{\mathbb{Z}} / \Gamma(p)}$ and $\operatorname{Mod}_{\mathrm{VB}_{\mathbb{F}_{p}}^{ \pm}}$. We now define an oriented version of the apartment monoid in $\operatorname{Mod}_{\mathrm{VB}_{\mathbb{F}_{p}}^{ \pm}}$. Recall that an orientation as in Example 5.4 is a generator of top exterior power of $X$ up to multiplication by \pm 1 . The oriented apartment monoid denoted $\overline{\mathbf{A}}^{ \pm}$is given on a vector space $X$ of dimension $n$ and orientation $\omega$ as follows. It is generated as a $\mathbf{k}$-module by symbols $\left[v_{1}, \ldots, v_{n}\right]$, one for each basis $v_{1}, \ldots, v_{n}$ of $X$ satisfying $\bigwedge_{i=1}^{n} v_{i}= \pm \omega$, subject to the following relations:

(a) $\left[v_{1}, \ldots, v_{n}\right]=\operatorname{sgn}(\sigma)\left[v_{\sigma(1)}, \ldots, v_{\sigma(n)}\right]$ for $\sigma$ a permutation.

(b) $\left[-v_{1}, v_{2}, \ldots, v_{n}\right]=\left[v_{1}, \ldots, v_{n}\right]$.

In other words, $\overline{\mathbf{A}}^{ \pm}$is the exterior algebra $\bigwedge\left(\operatorname{triv}_{1}\right)$ in $\operatorname{Mod}_{\mathrm{VB}_{\mathbb{F}_{p}}^{ \pm}}$where triv 1 is supported only in degree 1, and $\operatorname{triv}_{1}(1)$ is the trivial representation of $\mathbf{G L}_{1}^{ \pm}\left(\mathbb{F}_{p}\right)$. By Proposition 5.7, we know that the monoidal equivalence $\operatorname{Mod}_{\mathrm{VB}_{\mathbb{Z}} / \Gamma(p)} \rightarrow \operatorname{Mod}_{\mathrm{VB}_{\mathbb{F}_{p}}^{ \pm}}$takes $\mathbf{A}_{\Gamma(p)}$ to the oriented apartment monoid $\overline{\mathbf{A}}^{ \pm}$. By Borel-Serre duality, our conjecture for $i=0$ is the statement that the $\mathbf{G L}_{n}^{ \pm}\left(\mathbb{F}_{p}\right)$-equivariant map

$$
\operatorname{Ind}_{\mathbf{G} \mathbf{L}_{n-1}^{ \pm}\left(\mathbb{F}_{p}\right) \times \mathbf{G L}_{1}^{ \pm}\left(\mathbb{F}_{p}\right)} \mathrm{H}^{\left(\begin{array}{c}
n-1 \\
2
\end{array}\right)-j}\left(\Gamma_{n-1}(p) ; \mathbf{k}\right) \rightarrow \mathrm{H}^{\left(\begin{array}{c}
n \\
2
\end{array}\right)-j}\left(\Gamma_{n}(p) ; \mathbf{k}\right)
$$

is surjective for $n$ sufficiently large. In other words, the $i=0$ case of Conjecture 6.1 is the statement that the sequences

$$
\left\{\mathrm{H}^{\left(\begin{array}{l}
n \\
2
\end{array}\right)-j}\left(\Gamma_{n}(p) ; \mathbf{k}\right)\right\}_{\geq n}
$$

have finite generation degrees. Since $\mathbf{G L}_{n}^{ \pm}\left(\mathbb{F}_{3}\right)=\mathbf{G L}_{n}\left(\mathbb{F}_{3}\right)$, Theorem 1.1 establishes this conjecture for $i=0, j=1$, and $p=3$.

Since exterior algebras are Koszul, there is a Koszul resolution

$$
\overline{\mathbf{A}}^{ \pm} \otimes \operatorname{Sym}^{*}\left(\operatorname{triv}_{1}\right) \rightarrow \mathbf{k} \rightarrow 0 .
$$

This resolution can be used to calculate $\operatorname{Tor}_{i} \overline{\mathbf{A}}^{ \pm}(\mathbf{k}, M)$ for any $\overline{\mathbf{A}}^{ \pm}$-module $M$. In particular, if $\operatorname{deg} \operatorname{Tor}_{i}^{\overline{\mathbf{A}}^{ \pm}}(\mathbf{k}, M) \leq d$ for $i=0,1$ then the following sequence is exact in degrees $>d$ :

$$
M \otimes \operatorname{Sym}^{2}\left(\operatorname{triv}_{1}\right) \rightarrow M \otimes \operatorname{Sym}^{1}\left(\operatorname{triv}_{1}\right) \rightarrow M \rightarrow 0 .
$$

Concretely, if we think of $M$ as a sequence $\left\{M_{n}\right\}_{\geq 0}$ of representations of $\mathbf{G L}_{n}^{ \pm}\left(\mathbb{F}_{p}\right)$, then we have

$$
M_{n}=\operatorname{coker}\left(\operatorname{Ind}_{\mathbf{G L}_{n-2}^{ \pm}\left(\mathbb{F}_{p}\right) \times\left(\mathbb{Z} / 2 S_{2}\right)}^{\mathbf{L}_{n}^{ \pm}\left(\mathbb{F}_{p}\right)} M_{n-2} \otimes_{\mathbf{k}} \mathbf{k} \rightarrow \operatorname{Ind}_{\mathbf{G L}_{n-1}^{ \pm}\left(\mathbb{F}_{p}\right) \times \mathbf{G L}_{1}^{ \pm}\left(\mathbb{F}_{p}\right)}^{\mathbf{G L}_{ \pm-1}^{ \pm}\left(\mathbb{F}_{p}\right)} M_{n-\mathbf{k}} \mathbf{k}\right)
$$

for $n>d$. In particular, in a stable range, the representation of $M_{n}$ is determined by the representations $M_{n-1}$ and $M_{n-2}$ along with the stabilization map $M_{n-1} \rightarrow M_{n-2}$. This phenomenon is often called central stability [Put15, Pat] or finite presentation degree [CE17]. We shall refer 
to $d$ as the central stability degree of the sequence $\left\{M_{n}\right\}_{n \geq 0}$. We see that the conjecture for $i=0$ and 1 is the statement that the following sequence has finite central stability degree:

$$
\left\{\mathrm{H}^{\left(\begin{array}{c}
n \\
2
\end{array}\right)-j}\left(\Gamma_{n}(p) ; \mathbf{k}\right)\right\}_{n \geq 0} .
$$

We prove this for $j=0$ and all $p$ in the proposition below. We need a definition first. Given a prime field $R=\mathbb{F}_{p}$, we define the oriented Steinberg monoid $\overline{\mathbf{S t}}^{ \pm}=\mathbf{S t}_{\Gamma(p)}$. Using Bykovskii's presentation (see Theorem 2.5) the following presentation of $\overline{\mathbf{S t}}^{ \pm}$is immediate. On a vector space $X$ of dimension $n$ and orientation $\omega$ as follows, it is generated by as a $\mathbf{k}$-module by symbols $\left[v_{1}, \ldots, v_{n}\right]$, one for each basis $v_{1}, \ldots, v_{n}$ of $X$ satisfying $\bigwedge_{i=1}^{n} v_{i}= \pm \omega$, subject to the following relations:

(a) $\left[v_{1}, \ldots, v_{n}\right]=\operatorname{sgn}(\sigma)\left[v_{\sigma(1)}, \ldots, v_{\sigma(n)}\right]$ for $\sigma$ a permutation.

(b) $\left[-v_{1}, v_{2}, \ldots, v_{n}\right]=\left[v_{1}, \ldots, v_{n}\right]$.

(c) $\left[v_{1}, v_{2}, \ldots, v_{n}\right]-\left[v_{0}, v_{2}, \ldots, v_{n}\right]+\left[v_{0}, v_{1}, \ldots, v_{n}\right]=0$ where $v_{0}=v_{1}+v_{2}$.

Note that in $(b)$, we are only allowed to scale by -1 , not arbitrary units. This is what differentiates $\overline{\mathbf{S t}}_{n}^{ \pm}\left(\mathbb{F}_{p}\right)$ from $\mathbf{S t}_{n}\left(\mathbb{F}_{p}\right)$ for $p>3$. It is clear from this description that $\operatorname{deg} \operatorname{Tor}_{i} \overline{\mathbf{A}}^{ \pm}\left(\mathbf{k}, \overline{\mathbf{S t}}^{ \pm}\right) \leq 2 i$ for $i=0,1$. Thus the following sequence has central stability degree $\leq 2$ :

$$
\left\{\overline{\mathbf{S t}}_{n}^{ \pm}\left(\mathbb{F}_{p}\right)\right\}_{n \geq 0}=\left\{\mathrm{H}^{\mathrm{vcd}}\left(\Gamma_{n}(p) ; \mathbf{k}\right)\right\}_{n \geq 0} .
$$

Proposition 6.2. Let $p$ be an odd prime. Then $\operatorname{deg} \operatorname{Tor}_{i}^{\mathbf{A}_{\Gamma(p)}}\left(\mathbf{k}, \mathrm{H}_{0}(\Gamma(p) ; \mathbf{S t})\right) \leq 2 i$ for $i=0,1$. In other words, the sequence $\left\{\mathrm{H}^{\left(\begin{array}{c}n \\ 2\end{array}\right)}\left(\Gamma_{n}(p) ; \mathbf{k}\right)\right\}_{n \geq 0}$ has generation degree 0 and central stability degree $\leq 2$.

Proof. An argument similar to the one in Proposition 5.19, shows that the monoidal equivalence $\operatorname{Mod}_{\mathrm{VB}_{\mathbb{Z}} / \Gamma(p)} \rightarrow \operatorname{Mod}_{\mathrm{VB}_{\mathbb{F}_{p}}^{ \pm}}$takes $\mathbf{A}_{\Gamma(p)}$ to the oriented apartment monoid $\overline{\mathbf{A}}^{ \pm}$, and the $\mathbf{A}_{\Gamma(p)}$-module $\mathbf{S t}_{\Gamma(p)}$ to the $\overline{\mathbf{A}}^{ \pm}$-module $\overline{\mathbf{S t}}^{ \pm}$. The result now follows from the previous paragraph.

When $p=3$, we know by Lee-Szczarba's result [LS76b, Theorem 1.2] that $\mathbf{S t}_{\Gamma(p)} \cong \overline{\mathbf{S t}}$ (see Proposition 5.13 and Proposition 5.19). We see that

$$
\operatorname{Tor}_{i}^{\mathbf{A}_{\Gamma(p)}}\left(\mathbf{k}, \mathrm{H}_{0}(\Gamma(p) ; \mathbf{S t})\right) \cong \operatorname{Tor}_{i}^{\overline{\mathbf{A}}}(\mathbf{k}, \overline{\mathbf{S t}}) .
$$

Thus, Theorem 4.2 implies Conjecture 6.1 for $p=3, j=0$, and all $i$.

\section{Homological Vanishing for the Steinberg module}

In [APS18], Ash-Putman-Sam proved that $\mathrm{H}_{i}\left(\mathbf{G L}_{n}(K) ; \mathrm{St}_{n}(K)\right)$ vanishes for $K$ a field and $n$ sufficiently large compared with $i$. Similarly, Church-Putman [CP17] proved that $\mathrm{H}_{1}\left(\mathbf{G L}_{n}(\mathbb{Z}) ; \mathrm{St}_{n}(\mathbb{Z}) \otimes \mathbb{Q}\right)$ vanishes for all $n \geq 0$ and that $\mathrm{H}_{1}\left(\mathbf{S L}_{n}(\mathbb{Z}) ; \mathrm{St}_{n}(\mathbb{Z}) \otimes \mathbb{Q}\right)$ vanishes for $n \geq 3$. In this section, we give a new proof of the result of Ash-Putman-Sam and give integral refinements of these results of Church-Putman.

7.1. Homological vanishing for the Steinberg module of a field. Ash-Putman-Sam proved the following.

Theorem 7.1 (Ash-Putman-Sam [APS18]). Let $K$ be a field. Then $\mathrm{H}_{i}\left(\mathbf{G L}_{n}(K) ; \operatorname{St}_{n}(K) \otimes_{\mathbb{Z}} \mathbf{k}\right)=0$ for all $n \geq 2 i+2$. 
We use Koszulness of the Steinberg monoid (Theorem 3.2) to give a new proof of the theorem above. Unlike the original proof, our proof does not make use of high connectivity of the complex of partial bases. We will deduce homological vanishing for the Steinberg module using the following general criterion for homological vanishing for Koszul monoids which may be of independent interest. Note this is equivalent to Theorem 1.8 .

Theorem 7.2. Let $A$ be a (skew) commutative Koszul monoid in $\operatorname{Mod}_{\mathrm{VB}}$. Assume that $\mathbf{k}$ is a field such that the following holds:

(a) $\mathrm{H}_{0}\left(\mathbf{G L}_{2} ; A_{2}\right)=0$.

(b) The product map $\mathrm{H}_{0}\left(\mathbf{G L}_{1} ; A_{1}\right) \otimes \mathrm{H}_{1}\left(\mathbf{G L}_{2} ; A_{2}\right) \rightarrow \mathrm{H}_{1}\left(\mathbf{G L}_{3} ; A_{3}\right)$ is surjective.

Then we have that:

(a') The product map $\mathrm{H}_{0}\left(\mathbf{G L}_{1} ; A_{1}\right) \otimes \mathrm{H}_{i}\left(\mathbf{G L}_{n-1} ; A_{n-1}\right) \rightarrow \mathrm{H}_{i}\left(\mathbf{G L}_{n} ; A_{n}\right)$ is surjective for $n \geq 2 i+1$.

(b') $\mathrm{H}_{i}\left(\mathbf{G L}_{n} ; A_{n}\right)=0$ in degrees $n \geq 2 i+2$.

We shall refer to (a) and (b) in the theorem above as hypotheses or initial conditions.

Remark 7.3. As noted earlier, one can generalize our homological vanishing criterion for Koszul monoids replacing general linear groups with certain other families of groups. Our hypotheses (a) and (b) are likely necessary. In particular, they are necessary if one replaces general linear groups by symmetric groups and if one takes $A_{n}$ to be the sign representations. A result contributed to Pierre Vogel [Hau78, Proposition B] gives explicit calculations that show that the homology does not vanish in a slope $\frac{1}{2}$ range. We note that without Hypothesis (b), the homology still vanishes in a slope $\frac{1}{3}$ range. This can be proven by adapting the proof of Theorem 7.2. In particular, Hypothesis (a) implies that the product map $\mathrm{H}_{0}\left(\mathbf{G L}_{1} ; A_{1}\right) \otimes \mathrm{H}_{1}\left(\mathbf{G L}_{3} ; A_{3}\right) \rightarrow \mathrm{H}_{1}\left(\mathbf{G L}_{4} ; A_{4}\right)$ is surjective which can be used in place of Hypothesis (b).

We use the setup in $\S 5.1$ with $\mathcal{G}=\mathbf{G L}$ to prove the theorem. In this case, $\operatorname{Mod}_{\mathrm{VB} / \mathrm{GL}}$ is equivalent to $\operatorname{Mod}_{\mathbf{k}}^{\geq 0}$ - the category of non-negatively graded $\mathbf{k}$-modules.

Proposition 7.4. There is a spectral sequence of non-negatively graded $\mathbf{k}$-modules with

$$
E_{a b}^{1}=\mathrm{H}_{a}\left(\mathbf{G L} ; \overline{\mathcal{B}}_{b}(A)\right) \Longrightarrow \mathrm{L}_{a+b}\left(\mathrm{H}_{0}(\mathbf{G L} ;-) \circ\left(\mathbf{k} \otimes_{A}-\right)\right)(\mathbf{k}) .
$$

For A Koszul, we have that $E_{a b}^{\infty}$ is supported in degrees $\leq(a+b)$.

Proof. We have the following equality of the total derived functors

$$
\mathrm{L}\left(\mathrm{H}_{0}(\mathbf{G L} ;-) \circ\left(\mathbf{k} \otimes_{A}-\right)\right)(\mathbf{k})=\left(\mathrm{LH}_{0}(\mathbf{G L} ;-) \circ\left(\mathbf{k} \otimes_{A}^{\mathrm{L}}-\right)\right)(\mathbf{k})=\mathrm{LH}_{0}\left(\mathbf{G L} ; \mathbf{k} \otimes_{A}^{\mathrm{L}} \mathbf{k}\right)
$$

where the first equality holds because the hypotheses for the Grothendieck spectral sequence are satisfied. This involves noting that the functor $\mathbf{k} \otimes_{A}-: \operatorname{Mod}_{A} \rightarrow \operatorname{Mod}_{\mathrm{VB}}$ preserves projectives any projective $A$-module is of the form $A \otimes V$ where $V$ is a projective VB-module. Since the bar resolution for $A$ is acyclic with respect to the functor $\mathbf{k} \otimes_{A}-$, the total derived functor $\mathbf{k} \otimes_{A}^{\mathrm{L}} \mathbf{k}$ is represented by the complex $\overline{\mathcal{B}}_{*}(A)$ from $\S 3$. Thus there exists a spectral sequence given by

$$
E_{a b}^{1}=\mathrm{H}_{a}\left(\mathbf{G L} ; \overline{\mathcal{B}}_{b}(A)\right) \Longrightarrow \mathrm{L}_{a+b}\left(\mathrm{H}_{0}(\mathbf{G L} ;-) \circ\left(\mathbf{k} \otimes_{A}-\right)\right)(\mathbf{k}) .
$$


For the second statement, consider the Grothendieck spectral sequence

$$
{ }^{\prime} E_{a b}^{2}=\mathrm{H}_{a}\left(\mathbf{G L} ; \operatorname{Tor}_{b}^{A}(\mathbf{k}, \mathbf{k})\right) \Longrightarrow \mathrm{L}_{a+b}\left(\mathrm{H}_{0}(\mathbf{G L} ;-) \circ\left(\mathbf{k} \otimes_{A}-\right)\right)(\mathbf{k})
$$

as in the proof of Theorem 5.9. Since $A$ is Koszul, we see that ' $E_{a b}^{2}$ is supported in degrees $\leq(a+b)$. This shows that $E_{a b}^{\infty}$ is supported in degrees $\leq(a+b)$.

Lemma 7.5. Assume that $\mathbf{k}$ is a field.

(a) If Theorem 7.2 holds for $i \leq d$, then for any $a \leq d$ and $b>0$, we have

$$
\operatorname{deg} \mathrm{H}_{a}\left(\mathbf{G L} ; \overline{\mathcal{B}}_{b}(A)\right) \leq 2 a+b .
$$

(b) If Theorem 7.2 holds for $i \leq d$, then for any $0<a \leq d$ and $b>0$, the differential

$$
\mathrm{H}_{a}\left(\mathbf{G L} ; \overline{\mathcal{B}}_{b+1}(A)\right) \rightarrow \mathrm{H}_{a}\left(\mathbf{G L} ; \overline{\mathcal{B}}_{b}(A)\right)
$$

is surjective in degree $2 a+b$.

(c) If $b=0$, then we have $\operatorname{deg} \mathrm{H}_{a}\left(\mathbf{G L} ; \overline{\mathcal{B}}_{b}(A)\right) \leq 0$.

Proof. Proof of Part (a): By definition, we have

$$
\mathrm{H}_{a}\left(\mathbf{G L} ; \overline{\mathcal{B}}_{b}(A)\right)=\mathrm{H}_{a}\left(\mathbf{G L} ; A_{+}^{\otimes b}\right) .
$$

If $\mathbf{k}$ is a field, then by the Künneth formula, $\mathrm{H}_{a}\left(\mathbf{G L} ; A_{+}^{\otimes b}\right)$ in degree $n$ is a direct sum of $\mathbf{k}$-modules of the form

$$
\bigotimes_{j=1}^{b} \mathrm{H}_{a_{j}}\left(\mathbf{G} \mathbf{L}_{n_{j}} ; A_{n_{j}}\right)
$$

where $\sum_{j=1}^{b} a_{j}=a, \sum_{j=1}^{b} n_{j}=n$ and $n_{j}>0$ for all $j$. If $n>2 a+b$, then by the pigeonhole principle we have $n_{j} \geq 2 a_{j}+2$ for some $j$. Thus we have

$$
\bigotimes_{j=1}^{b} \mathrm{H}_{a_{j}}\left(\mathbf{G L}_{n_{j}} ; A_{n_{j}}\right)=0
$$

completing the proof of Part (a).

Proof of Part (b): By the pigeonhole principle, $\mathrm{H}_{a}\left(\mathbf{G L} ; A_{+}^{\otimes b}\right)$ in degree $2 a+b$ is a direct sum of k-modules of the form

$$
\bigotimes_{j=1}^{b} \mathrm{H}_{a_{j}}\left(\mathbf{G L}_{n_{j}} ; A_{n_{j}}\right)
$$

where $\sum_{j=1}^{b} a_{j}=a, n_{j}=2 a_{j}+1$ for all $j$. We say that such a direct summand is of type $j_{0}$ if $j_{0}$ is the largest such that $n_{j_{0}} \geq 2$. This must exist as $a>0$. For a type $j_{0}$ direct summand as above, define $a_{j}^{\prime}$ and $n_{j}^{\prime}$ for $1 \leq j \leq b+1$ by

$$
\left(a_{j}^{\prime}, n_{j}^{\prime}\right)= \begin{cases}\left(a_{j}, n_{j}\right) & \text { if } j<j_{0} \\ (0,1) & \text { if } j=j_{0} \\ \left(a_{j_{0}}, n_{j_{0}}-1\right) & \text { if } j=j_{0}+1 \\ \left(a_{j-1}, n_{j-1}\right) & \text { if } j>j_{0}+1 .\end{cases}
$$


Then

$$
\bigotimes_{j=1}^{b+1} \mathrm{H}_{a_{j}^{\prime}}\left(\mathbf{G L}_{n_{j}^{\prime}} ; A_{n_{j}^{\prime}}\right)
$$

is a direct summand of $\mathrm{H}_{a}\left(\mathbf{G L} ; \overline{\mathcal{B}}_{b+1}(A)\right)$ and the differential is given by the alternating sum of multiplying two of the consecutive factors. Since the theorem holds for $a \leq d$, we have the following:

- Multiplication of any of these consecutive factors besides $j_{0}$-th and $\left(j_{0}+1\right)$-th factors, or $\left(j_{0}+1\right)$-th and $\left(j_{0}+2\right)$-th factors is zero.

- Multiplying $j_{0}$-th and $\left(j_{0}+1\right)$-th factors yields a surjective map

$$
\bigotimes_{j=1}^{b+1} \mathrm{H}_{a_{j}^{\prime}}\left(\mathbf{G L}_{n_{j}^{\prime}} ; A_{n_{j}^{\prime}}\right) \rightarrow \bigotimes_{j=1}^{b} \mathrm{H}_{a_{j}}\left(\mathbf{G L}_{n_{j}} ; A_{n_{j}}\right) \text {. }
$$

- Multiplying $\left(j_{0}+1\right)$-th and $\left(j_{0}+2\right)$-th factors takes

$$
\bigotimes_{j=1}^{b+1} \mathrm{H}_{a_{j}^{\prime}}\left(\mathbf{G L}_{n_{j}^{\prime}} ; A_{n_{j}^{\prime}}\right)
$$

inside a direct summand of type $>j_{0}$.

Let Type ejo $_{0}$ denote the direct sum of summands of type $j$ for $j \geq j_{0}$. Our argument above shows, by an easy reverse induction on $j_{0}$, that the image of the differential

$$
\mathrm{H}_{a}\left(\mathbf{G L} ; \overline{\mathcal{B}}_{b+1}(A)\right) \rightarrow \mathrm{H}_{a}\left(\mathbf{G L} ; \overline{\mathcal{B}}_{b}(A)\right),
$$

in degree $2 a+b$, contains Type $\geq j_{0}$ for any $j_{0}$. Thus the differential is surjective, completing the proof of Part (b).

Proof of Part (c): Part (c) is immediate from $A_{+}^{\otimes 0}=\mathbf{k}$.

We now use this lemma to prove our homological vanishing criterion for Koszul monoids.

Proof of Theorem 7.2. The base case $i=-1$ is trivial. Assume now that $i \geq 0$ and that the theorem holds for $a<i$. Consider the spectral sequence

$$
E_{a b}^{1}=\mathrm{H}_{a}\left(\mathbf{G L} ; \overline{\mathcal{B}}_{b}(A)\right) \Longrightarrow \mathrm{L}_{a+b}\left(\mathrm{H}_{0}(\mathbf{G L} ;-) \circ\left(\mathbf{k} \otimes_{A}-\right)\right)(\mathbf{k})
$$

as in Proposition 7.4, and assume that $n \geq 2 i+1$. In the following two paragraphs, we analyze this spectral sequence of graded k-modules on the diagonals $a+b=i+1$ and $a+b=i+2$ in degree $n$.

Assume that $a+b=i+1$. If $a<i$, then by induction and Part (a) of the previous lemma, we have

$$
\operatorname{deg} \mathrm{H}_{a}\left(\mathbf{G L} ; \overline{\mathcal{B}}_{b}(A)\right) \leq 2 a+b=2(i+1)-b \leq 2 i<n .
$$

Moreover if $a=i+1$, then by Part (c) of the previous lemma, we have $\operatorname{deg} \mathrm{H}_{a}\left(\mathbf{G L} ; \overline{\mathcal{B}}_{b}(A)\right)<n$. In particular, the only nonzero entry in degree $n$ on the diagonal $a+b=i+1$ comes from $E_{i, 1}^{1}$.

Assume that $a+b=i+2$. If $a<i-1$, then by Part (a) of the previous lemma, we have

$$
\operatorname{deg} \mathrm{H}_{a}\left(\mathbf{G L} ; \overline{\mathcal{B}}_{b}(A)\right) \leq 2 a+b=2(i+2)-b=2 i+4-b<n .
$$

If $a=i-1$ and $n>2 i+1$, then by Part (a) of the previous lemma, the differential

$$
\mathrm{H}_{i-1}\left(\mathbf{G L} ; \overline{\mathcal{B}}_{4}(A)\right) \rightarrow \mathrm{H}_{i-1}\left(\mathbf{G L} ; \overline{\mathcal{B}}_{3}(A)\right)
$$


is surjective in degree $n$ as the target vanishes. If $a=i-1>0$ and $n=2 i+1$, then by Part (b) of the previous lemma, the differential

$$
\mathrm{H}_{i-1}\left(\mathbf{G L} ; \overline{\mathcal{B}}_{4}(A)\right) \rightarrow \mathrm{H}_{i-1}\left(\mathbf{G L} ; \overline{\mathcal{B}}_{3}(A)\right)
$$

is surjective in degree $n$. In the remaining case, $a=i-1=0, n=2 i+1$, and the differential

$$
\mathrm{H}_{i-1}\left(\mathbf{G L} ; \overline{\mathcal{B}}_{4}(A)\right) \rightarrow \mathrm{H}_{i-1}\left(\mathbf{G L} ; \overline{\mathcal{B}}_{3}(A)\right)
$$

may not be surjective in degree $n$, and that is why we need an additional hypothesis, namely Hypothesis (b), in the case $i=1$.

We now use the paragraphs above to show that the differential

$$
E_{i, 2}^{1}=\mathrm{H}_{i}\left(\mathbf{G L} ; A_{+}^{\otimes 2}\right) \rightarrow \mathrm{H}_{i}\left(\mathbf{G L} ; A_{+}\right)=E_{i, 1}^{1}
$$

is surjective in degree $n$. The case $i=0$ and $n=1$ follows from Hypothesis (a). In the remaining cases, we have $n \geq i+2$. Since $E_{a b}^{\infty}$ is supported in degrees $\leq(a+b)$, we see that $E_{a b}^{\infty}$ vanishes in degree $n$ on the line $a+b=i+1$. Since $n \geq i+2$, some differential at some page must kill the degree $n$ piece of the entry $E_{i, 1}^{1}$. By the paragraph above, we have $E_{a b}^{2}=0$ in degree $n$ for $a+b=i+2$ for $a \leq i-1$ except when $n=2 i+1=i+2$. In this exceptional case, our claim follows from Hypothesis (b). Away from this exceptional case, we have $E_{a b}^{2}=0$ in degree $n$ for $a+b=i+2$ and $a \leq i-1$. Since $E_{i, 1}^{\infty}=0$, we must have that

$$
E_{i, 2}^{1}=\mathrm{H}_{i}\left(\mathbf{G L} ; A_{+}^{\otimes 2}\right) \rightarrow \mathrm{H}_{i}\left(\mathbf{G L} ; A_{+}\right)=E_{i, 1}^{1}
$$

is surjective in degree $n$, proving our claim.

By induction, the theorem holds for $a<i$. Hence the degree $n$ piece of $\mathrm{H}_{i}\left(\mathbf{G L} ; A_{+}^{\otimes 2}\right)$ is given by

$$
\mathrm{H}_{i}\left(\mathbf{G L}_{n-1} ; A_{n-1}\right) \otimes \mathrm{H}_{0}\left(\mathbf{G L}_{1} ; A_{1}\right) \bigoplus \mathrm{H}_{0}\left(\mathbf{G L}_{1} ; A_{1}\right) \otimes \mathrm{H}_{i}\left(\mathbf{G L}_{n-1} ; A_{n-1}\right) .
$$

By the previous paragraph and the (skew) commutativity of $A$, we conclude that

$$
\mathrm{H}_{0}\left(\mathbf{G L}_{1} ; A_{1}\right) \otimes \mathrm{H}_{i}\left(\mathbf{G L}_{n-1} ; A_{n-1}\right) \rightarrow \mathrm{H}_{i}\left(\mathbf{G L}_{n} ; A_{n}\right)
$$

is surjective for $n \geq 2 i+1$. This proves Part (a').

For Part (b'), note that

$$
\mathrm{H}_{0}\left(\mathbf{G L}_{1} ; A_{1}\right) \otimes \mathrm{H}_{0}\left(\mathbf{G L}_{1} ; A_{1}\right) \otimes \mathrm{H}_{i}\left(\mathbf{G L}_{n-2} ; A_{n-2}\right) \rightarrow \mathrm{H}_{i}\left(\mathbf{G L}_{n} ; A_{n}\right)
$$

is surjective by Part (a'). But, by associativity of multiplication, this map factors through

$$
\mathrm{H}_{0}\left(\mathbf{G L}_{2} ; A_{2}\right) \otimes \mathrm{H}_{i}\left(\mathbf{G L}_{n-2} ; A_{n-2}\right) \rightarrow \mathrm{H}_{i}\left(\mathbf{G L}_{n} ; A_{n}\right),
$$

which vanishes by Hypothesis (a). This completes the proof.

We now show that the initial conditions in Theorem 7.2 are satisfied when $A=$ St. Let $T=$ $\mathrm{T}\left(\mathrm{reg}_{1}\right)$ be the algebra as in Proposition 3.8 for $R=K$. Then $\mathrm{H}_{0}(\mathbf{G L} ; T)$ is naturally isomorphic to the polynomial ring $\mathbf{k}[t]$ which is a monoid in $\operatorname{Mod}_{\mathbf{k}}^{\geq 0}$. Thus $\mathrm{H}_{0}(\mathbf{G L} ;-)$ induces a functor

$$
\mathrm{H}_{0}^{T}(\mathbf{G L} ;-): \operatorname{Mod}_{T} \rightarrow \operatorname{Mod}_{\mathbf{k}[t]} .
$$


Note that there are natural surjections $T \rightarrow \mathbf{S t} \rightarrow \mathbf{k}$ of monoids, and so $\mathbf{S t}$ is a module over $T$. Note here that Proposition 4.6 is not applicable as $T$ is not (skew) commutative. Now we apply the Grothendieck spectral sequence from the proof of Theorem 5.9 to the $T$-module St to obtain:

$$
E_{a b}^{2}=\operatorname{Tor}_{a}^{\mathbf{k}[t]}\left(\mathbf{k}, \mathrm{H}_{b}^{T}(\mathbf{G L} ; \mathbf{S t})\right) \Longrightarrow \mathrm{L}_{a+b}\left(\left(\mathbf{k} \otimes_{\mathbf{k}[t]}-\right) \circ \mathrm{H}_{0}^{T}(\mathbf{G L} ;-)\right)(\mathbf{S t}) .
$$

A version of Lemma 5.10 for $T$, implies that $\mathrm{H}_{q}^{T}(\mathbf{G L} ;-)$ is isomorphic to $\mathrm{H}_{q}(\mathbf{G L} ;-)$ as a graded k-module and so we shall now drop the superscript $T$ from our notation. Note that $\mathrm{H}_{q}(\mathbf{G L} ;-)$ has the structure of a $\mathbf{k}[t]$-module. The spectral sequence above simplifies to

$$
E_{a b}^{2}=\operatorname{Tor}_{a}^{\mathbf{k}[t]}\left(\mathbf{k}, \mathrm{H}_{b}(\mathbf{G L} ; \mathbf{S t})\right) \Longrightarrow \mathrm{L}_{a+b}\left(\left(\mathbf{k} \otimes_{\mathbf{k}[t]}-\right) \circ \mathrm{H}_{0}(\mathbf{G L} ;-)\right)(\mathbf{S t}) .
$$

Lemma 7.6. If $\mathrm{L}_{1}\left(\left(\mathbf{k} \otimes_{\mathbf{k}[t]}-\right) \circ \mathrm{H}_{0}(\mathbf{G L} ;-)\right)(\mathbf{S t})$ vanishes in degree $d$, then the product map

$$
\mathrm{H}_{0}\left(\mathbf{G L}_{1} ; \mathbf{S t}_{1}\right) \otimes \mathrm{H}_{1}\left(\mathbf{G L}_{d-1} ; \mathbf{S t}_{d-1}\right) \rightarrow \mathrm{H}_{1}\left(\mathbf{G L}_{d} ; \mathbf{S t}_{d}\right)
$$

is surjective.

Proof. Since $\operatorname{Tor}_{i}^{\mathbf{k}[t]}=0$ for $i>1$, we have $E_{a b}^{2}=E_{a b}^{\infty}$. Thus, if $\mathrm{L}_{1}\left(\left(\mathbf{k} \otimes_{\mathbf{k}[t]}-\right) \circ \mathrm{H}_{0}(\mathbf{G L} ;-)\right)(\mathbf{S t})$ vanishes in degree $d$, then $E_{0,1}^{2}$ vanishes in degree $d$. Since $\mathbf{k}[t]=\mathrm{H}_{0}(\mathbf{G L} ; T)$, this is equivalent to the surjectivity of the product map

$$
\mathrm{H}_{0}\left(\mathbf{G L}_{1} ; T_{1}\right) \otimes \mathrm{H}_{1}\left(\mathbf{G L}_{d-1} ; \mathbf{S t}_{d-1}\right) \rightarrow \mathrm{H}_{1}\left(\mathbf{G L}_{d} ; \mathbf{S t}_{d}\right) .
$$

The assertion now follows from Proposition 5.12 Part (b) applied to $A=T$ and $B=\mathbf{S t}$.

To calculate $\mathrm{L}_{1}\left(\left(\mathbf{k} \otimes_{\mathbf{k}[t]}-\right) \circ \mathrm{H}_{0}(\mathbf{G L} ;-)\right)(\mathbf{S t})$, we need a resolution of $\mathbf{S t}$ by projective $T$-modules. The Lee-Szczarba resolution $\mathrm{LS}_{*}$ is a such a resolution.

Let $X$ be an object in $\mathrm{VB}=\mathrm{VB}_{K}$ of rank $n$. Let $\mathrm{LS}_{i}$ be the VB-module defined as follows. The module $\mathrm{LS}_{i}(X)$ is a $\mathbf{k}$-module generated by symbols of the form $\left[v_{1}, \ldots, v_{n+i}\right]$ where the $v_{j}$ are distinct nonzero elements of $X$ and where we impose the following relation:

$$
\left[v_{1}, \ldots, v_{n+i}\right]=0 \text { if } v_{1}, \ldots, v_{n} \text { do not } \operatorname{span} X .
$$

There is an equivariant map

$$
T\left(X_{1}\right) \otimes \mathrm{LS}_{i}\left(X_{2}\right) \rightarrow \mathrm{LS}_{i}(X)
$$

given by

$$
\left[u_{1}, \ldots, u_{k}\right]\left[v_{1}, \ldots, v_{n-k+i}\right]=\left[u_{1}, \ldots, u_{k}, v_{1}, \ldots, v_{n-k+i}\right] .
$$

This gives $\mathrm{LS}_{i}$ the structure of a $T$-module. It is easy to see that $\mathrm{LS}_{i}$ is a projective $T$-module. There is a natural differential $\mathrm{LS}_{i} \rightarrow \mathrm{LS}_{i-1}$ given by the alternating sum of forgetting vectors. By [LS76b, Theorem 3.1], $\mathrm{LS}_{*} \rightarrow \mathbf{S t}$ is a $T$-projective resolution. In particular, we have

$$
\mathrm{L}_{i}\left(\left(\mathbf{k} \otimes_{\mathbf{k}[t]}-\right) \circ \mathrm{H}_{0}(\mathbf{G L} ;-)\right)(\mathbf{S t})=\mathrm{H}_{i}\left(\mathbf{k} \otimes_{\mathbf{k}[t]} \mathrm{H}_{0}\left(\mathbf{G L} ; \mathrm{LS}_{*}\right)\right) .
$$

Since $\operatorname{deg} \mathbf{k} \otimes_{\mathbf{k}[t]} \mathrm{H}_{0}\left(\mathbf{G L} ; \mathrm{LS}_{0}\right)=0$, any element of $\mathbf{k} \otimes_{\mathbf{k}[t]} \mathrm{H}_{0}\left(\mathbf{G L} ; \mathrm{LS}_{1}\right)$ in positive degrees is a cycle. Thus to show that $\mathrm{L}_{1}\left(\left(\mathbf{k} \otimes_{\mathbf{k}[t]}-\right) \circ \mathrm{H}_{0}(\mathbf{G L} ;-)\right)(\mathbf{S t})$ vanishes in degree 3 , it suffices to show that the following map is surjective in degree 3 :

$$
d_{2}: \mathbf{k} \otimes_{\mathbf{k}[t]} \mathrm{H}_{0}\left(\mathbf{G L} ; \mathrm{LS}_{2}\right) \rightarrow \mathbf{k} \otimes_{\mathbf{k}[t]} \mathrm{H}_{0}\left(\mathbf{G L} ; \mathrm{LS}_{1}\right) .
$$

Recall that a minimal linearly dependent subset of a vector space is called a circuit. 
Lemma 7.7. $\mathrm{L}_{1}\left(\left(\mathbf{k} \otimes_{\mathbf{k}[t]}-\right) \circ \mathrm{H}_{0}(\mathbf{G L} ;-)\right)(\mathbf{S t})$ vanishes in degree 3 .

Proof. Let $v_{1}, v_{2}, v_{3}, v_{4}$ be nonzero vectors in $K^{3}$. By the previous paragraph, it suffices to show that the image of $\left[v_{1}, v_{2}, v_{3}, v_{4}\right]$ in coker $d_{2}$ vanishes. We do this case-by-case as follows.

Case (a). $\left\{v_{2}, v_{3}, v_{4}\right\}$ is not a basis of $K^{3}$.

We can assume without loss of generality that $\left\{v_{1}, v_{2}, v_{3}, v_{4}\right\}$ spans $K^{3}$. The hypothesis implies that $v_{1}$ does not lie in the span of $\left\{v_{2}, v_{3}, v_{4}\right\}$. Thus we have

$$
\left[v_{1}, v_{2}, v_{3}, v_{4}\right]=\left[v_{1}\right]\left[v_{2}, v_{3}, v_{4}\right] \in T_{+} \mathrm{LS}_{1},
$$

and so its image in $\mathrm{H}_{0}\left(\mathbf{G L} ; \mathrm{LS}_{1}\right)$ is contained in $\mathbf{k}[t]_{+} \mathrm{H}_{0}\left(\mathbf{G L} ; \mathrm{LS}_{1}\right)$. The assertion follows from this.

Case (b). $\left\{v_{1}, v_{2}, v_{3}, v_{4}\right\}$ is a circuit.

Note that we have

$$
\begin{aligned}
d_{2}\left(\left[v_{1}, v_{2}, v_{3}+v_{4}, v_{3}, v_{4}\right]\right) & =\left[v_{2}, v_{3}+v_{4}, v_{3}, v_{4}\right]-\left[v_{1}, v_{3}+v_{4}, v_{3}, v_{4}\right]+\left[v_{1}, v_{2}, v_{3}, v_{4}\right] \\
& -\left[v_{1}, v_{2}, v_{3}+v_{4}, v_{4}\right]+\left[v_{1}, v_{2}, v_{3}+v_{4}, v_{3}\right] .
\end{aligned}
$$

By Case (a), the first two terms vanish in coker $d_{2}$. Let $g \in \mathbf{G L}_{3}(K)$ be the linear involution which fixes $v_{1}$ and $v_{2}$ and takes $v_{3}$ to $v_{4}$. Then $g$ interchanges the last two terms in the expression above, and so the last two terms cancel each other out in coker $d_{2}$. Thus the middle term vanishes in coker $d_{2}$, completing the proof in Case (b).

Case (c). $\left\{v_{2}, v_{3}, v_{4}\right\}$ is a basis of $K^{3}$ and $v_{1}$ is a constant multiple of either $v_{2}, v_{3}$, or $v_{4}$.

Let $u=v_{2}+v_{3}+v_{4}$. Then we have

$$
d_{2}\left(\left[u, v_{1}, v_{2}, v_{3}, v_{4}\right]\right)=\left[v_{1}, v_{2}, v_{3}, v_{4}\right]-\left[u, v_{2}, v_{3}, v_{4}\right]+\left[u, v_{1}, v_{3}, v_{4}\right]-\left[u, v_{1}, v_{2}, v_{4}\right]+\left[u, v_{1}, v_{2}, v_{3}\right] .
$$

Each of the last four terms fall under Case (a) or Case (b), and so the images of the last four terms vanish in coker $d_{2}$. Thus the image of the first term vanishes in coker $d_{2}$, completing the proof in Case (c).

Case (d). $\left\{v_{2}, v_{3}, v_{4}\right\}$ is a basis of $K^{3}$ and $v_{1}=a_{2} v_{2}+a_{3} v_{3}+a_{4} v_{4}$ such that exactly one of $a_{2}, a_{3}, a_{4}$ is 0 .

The hypothesis implies that there exists a unique $i \in\{2,3,4\}$ such that $a_{i}=0$. Set $u=v_{i}$. Then we have

$$
d_{2}\left(\left[u, v_{1}, v_{2}, v_{3}, v_{4}\right]\right)=\left[v_{1}, v_{2}, v_{3}, v_{4}\right]-\left[u, v_{2}, v_{3}, v_{4}\right]+\left[u, v_{1}, v_{3}, v_{4}\right]-\left[u, v_{1}, v_{2}, v_{4}\right]+\left[u, v_{1}, v_{2}, v_{3}\right] .
$$

For each of the last four terms, if $v_{i}$ is present in it then the term falls under Case (c), and if $v_{i}$ is not present in it then the term falls under Case (a). Thus the last four terms vanish in coker $d_{2}$. This shows that the first term vanish in coker $d_{2}$, completing the proof in Case (c).

The four cases above are exhaustive, and so our proof is complete.

Proof of Theorem 7.1. It suffices to prove the theorem when $\mathbf{k}$ is a field as the general case follows from the field case via the universal coefficient theorem. By Theorem 3.2, we know that St is Koszul. Moreover, we have $\mathbf{S t}_{n}(K)=\mathrm{St}_{n}(K) \otimes_{\mathbb{Z}} \mathbf{k}$. Thus it suffices to verify Hypotheses (a) and (b) in Theorem 7.2. Hypothesis (a) follows from [APS18, Lemma 2.6], and Hypothesis (b) follows from Lemma 7.6 and Lemma 7.7. 
7.2. Homological vanishing for the Steinberg module of the integers. We now prove integral refinements of Church-Putman's homological vanishing theorem (Theorem 1.9) for the Steinberg module of the integers.

Theorem 7.8. Let $\mathbf{k}$ be an arbitrary commutative ring. For $n \geq 6$, we have that:

$$
\begin{aligned}
\mathrm{H}_{1}\left(\mathbf{G L}_{n}(\mathbb{Z}) ; \mathrm{St}_{n}(\mathbb{Z}) \otimes_{\mathbb{Z}} \mathbf{k}\right) & =0, \\
\mathrm{H}_{1}\left(\mathbf{S L}_{n}(\mathbb{Z}) ; \mathrm{St}_{n}(\mathbb{Z}) \otimes_{\mathbb{Z}} \mathbf{k}\right) & =0 .
\end{aligned}
$$

Proof. It suffices to prove the assertion for SL. The Lyndon-Hochschild-Serre spectral sequence corresponding to the short exact sequence

$$
1 \rightarrow \Gamma_{n}(3) \rightarrow \mathbf{S L}_{n}(\mathbb{Z}) \rightarrow \mathbf{S L}_{n}\left(\mathbb{F}_{3}\right) \rightarrow 1
$$

is given by

$$
\mathrm{H}_{p}\left(\mathbf{S L}_{n}\left(\mathbb{F}_{3}\right) ; \mathrm{H}_{q}\left(\Gamma_{n}(3) ; \mathrm{St}_{n}(\mathbb{Z}) \otimes_{\mathbb{Z}} \mathbf{k}\right)\right) \Longrightarrow \mathrm{H}_{p+q}\left(\mathbf{S L}_{n}(\mathbb{Z}) ; \mathrm{St}_{n}(\mathbb{Z}) \otimes_{\mathbb{Z}} \mathbf{k}\right)
$$

Thus it suffices to show that

$$
\begin{array}{r}
\mathrm{H}_{1}\left(\mathbf{S L}_{n}\left(\mathbb{F}_{3}\right) ; \mathrm{H}_{0}\left(\Gamma_{n}(3) ; \mathrm{St}_{n}(\mathbb{Z}) \otimes_{\mathbb{Z}} \mathbf{k}\right)\right), \\
\mathrm{H}_{0}\left(\mathbf{S L}_{n}\left(\mathbb{F}_{3}\right) ; \mathrm{H}_{1}\left(\Gamma_{n}(3) ; \operatorname{St}_{n}(\mathbb{Z}) \otimes_{\mathbb{Z}} \mathbf{k}\right)\right)
\end{array}
$$

vanish for $n \geq 6$. By [LS76b, Theorem 1.2], we know that $\mathrm{H}_{0}\left(\Gamma_{n}(3) ; \mathrm{St}_{n}(\mathbb{Z}) \otimes_{\mathbb{Z}} \mathbf{k}\right)=\mathrm{St}_{n}\left(\mathbb{F}_{3}\right) \otimes_{\mathbb{Z}} \mathbf{k}$. Thus $\mathrm{H}_{1}\left(\mathbf{S L}_{n}\left(\mathbb{F}_{3}\right) ; \mathrm{H}_{0}\left(\Gamma_{n}(3) ; \mathrm{St}_{n}(\mathbb{Z}) \otimes_{\mathbb{Z}} \mathbf{k}\right)\right)=0$ for $n \geq 4$ by the homological vanishing result of Ash-Putman-Sam ([APS18, Theorem 1.1]).

Let $\overline{\mathbf{S t}}$ be the Steinberg monoid in $\operatorname{Mod}_{\mathrm{VB}_{\mathbb{F}_{3}}}$. By Proposition 5.19 and Corollary 5.15, $\mathrm{H}_{1}(\Gamma(3), \mathbf{S t})$ is an $\overline{\mathbf{S t}}$-module generated in degrees $\leq 4$. In particular, if $n \geq 4$ then we have a surjection

$$
\operatorname{Ind}_{\mathbf{G L}_{n-4}\left(\mathbb{F}_{3}\right) \times \mathbf{G} \mathbf{L}_{4}\left(\mathbb{F}_{3}\right)}^{\mathbf{G L}_{n}\left(\mathbb{F}_{3}\right)} \mathbf{S t}_{n-4}\left(\mathbb{F}_{3}\right) \otimes_{\mathbf{k}} \mathrm{H}_{1}\left(\Gamma_{4}(3) ; \mathbf{S t}_{4}(\mathbb{Z})\right) \rightarrow \mathrm{H}_{1}\left(\Gamma_{n}(3) ; \mathbf{S t}_{n}(\mathbb{Z})\right) .
$$

Now if $n \geq 6$, then $n-4 \geq 2$, and so by [APS18, Lemma 2.6] we have $\mathrm{H}_{0}\left(\mathbf{S L}_{n-4}\left(\mathbb{F}_{3}\right) ; \mathbf{S t}_{n-4}\left(\mathbb{F}_{3}\right)\right)=0$. This shows that $\mathrm{H}_{0}\left(\mathbf{S L}_{n}\left(\mathbb{F}_{3}\right) ; \mathrm{H}_{1}\left(\Gamma_{n}(3) ; \mathbf{S t}_{n}(\mathbb{Z})\right)\right)=0$ for $n \geq 6$. This completes the proof after noting that $\operatorname{St}_{n}(\mathbb{Z}) \otimes_{\mathbb{Z}} \mathbf{k}=\mathbf{S t}_{n}(\mathbb{Z})$.

\section{REFERENCES}

[AGM02] Avner Ash, Paul E. Gunnells, and Mark McConnell, Cohomology of congruence subgroups of $\mathrm{SL}_{4}(\mathbb{Z}), \mathrm{J}$. Number Theory 94 (2002), no. 1, 181-212. MR 1904968

[AGM08] _ Cohomology of congruence subgroups of $\mathrm{SL}(4, \mathbb{Z})$. II, J. Number Theory 128 (2008), no. 8, 2263-2274. MR 2394820

[AGM10] Cohomology of congruence subgroups of $\mathrm{SL}_{4}(\mathbb{Z})$. III, Math. Comp. 79 (2010), no. 271, $1811-1831$. MR 2630015

[APS18] Avner Ash, Andrew Putman, and Steven V. Sam, Homological vanishing for the Steinberg representation, Compos. Math. 154 (2018), no. 6, 1111-1130. MR 3797603

[AR79] Avner Ash and Lee Rudolph, The modular symbol and continued fractions in higher dimensions, Invent. Math. 55 (1979), no. 3, 241-250. MR 553998

[Ash94] Avner Ash, Unstable cohomology of $S L(n, O)$, J. Algebra 167 (1994), no. 2, 330-342. MR 1283290

[BC07] Maurizio Brunetti and Adriana Ciampella, A Priddy-type Koszulness criterion for non-locally finite algebras, Colloq. Math. 109 (2007), no. 2, 179-192. MR 2318516 
[Bor74] Armand Borel, Stable real cohomology of arithmetic groups, Ann. Sci. École Norm. Sup. (4) 7 (1974), 235-272 (1975). MR 0387496

[Bro89] Kenneth S. Brown, Buildings, Springer-Verlag, New York, 1989. MR 969123

[BS73] A. Borel and J.-P. Serre, Corners and arithmetic groups, Comment. Math. Helv. 48 (1973), 436-491, Avec un appendice: Arrondissement des variétés à coins, par A. Douady et L. Hérault. MR 0387495

[Byk03] V. A. Bykovskiı, Generating elements of the annihilating ideal for modular symbols, Funktsional. Anal. i Prilozhen. 37 (2003), no. 4, 27-38, 95. MR 2083229

[Cal15] Frank Calegari, The stable homology of congruence subgroups, Geom. Topol. 19 (2015), no. 6, 3149-3191. MR 3447101

[CE17] Thomas Church and Jordan Ellenberg, Homology of FI-modules, Geometry \& Topology 21 (2017), no. 4, $2373-2418$.

[CEF15] Thomas Church, Jordan S. Ellenberg, and Benson Farb, FI-modules and stability for representations of symmetric groups, Duke Math. J. 164 (2015), no. 9, 1833-1910. MR 3357185

[CEFN14] Thomas Church, Jordan S. Ellenberg, Benson Farb, and Rohit Nagpal, FI-modules over Noetherian rings, Geom. Topol. 18 (2014), no. 5, 2951-2984. MR 3285226

[CFP14] Thomas Church, Benson Farb, and Andrew Putman, A stability conjecture for the unstable cohomology of $\mathrm{SL}_{n} \mathbb{Z}$, mapping class groups, and $\operatorname{Aut}\left(F_{n}\right)$, Algebraic topology: applications and new directions, Contemp. Math., vol. 620, Amer. Math. Soc., Providence, RI, 2014, pp. 55-70. MR 3290086

[CFP19] _ Integrality in the Steinberg module and the top-dimensional cohomology of $\mathrm{SL}_{n} \mathcal{O}_{K}$, Amer. J. Math. 141 (2019), no. 5, 1375-1419. MR 4011804

[Cha84] Ruth Charney, On the problem of homology stability for congruence subgroups, Comm. Algebra 12 (1984), no. 17-18, 2081-2123. MR 747219

[CMNR18] Thomas Church, Jeremy Miller, Rohit Nagpal, and Jens Reinhold, Linear and quadratic ranges in representation stability, Advances in Mathematics 333 (2018), 1 - 40.

[CP17] Thomas Church and Andrew Putman, The codimension-one cohomology of $\mathrm{SL}_{n} \mathbb{Z}$, Geom. Topol. 21 (2017), no. 2, 999-1032. MR 3626596

[CS16] Marc Chardin and Peter Symonds, Degree bounds on homology and a conjecture of Derksen, Compos. Math. 152 (2016), no. 10, 2041-2049. MR 3569999

[Dja] Aurélien Djament, De l'homologie stable des groupes de congruence, preprint, https://hal . archives-ouvertes.fr/hal-01565891v2.

[DSGG $\left.{ }^{+} 19\right]$ Mathieu Dutour Sikirić, Herbert Gangl, Paul E. Gunnells, Jonathan Hanke, Achill Schürmann, and Dan Yasaki, On the topological computation of $K_{4}$ of the Gaussian and Eisenstein integers, J. Homotopy Relat. Struct. 14 (2019), no. 1, 281-291. MR 3913976

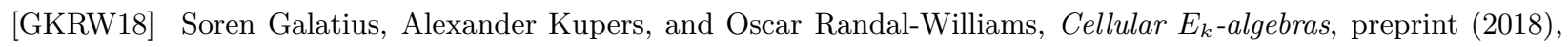
http://arxiv.org/abs/1805.07184.

[GL19] Wee Liang Gan and Liping Li, Linear stable range for homology of congruence subgroups via FI-modules, Selecta Math. (N.S.) 25 (2019), no. 4, Art. 55, 11. MR 3997138

[Hau78] Jean-Claude Hausmann, Manifolds with a given homology and fundamental group, Comment. Math. Helv. 53 (1978), no. 1, 113-134. MR 483534

[LS76a] Ronnie Lee and R. H. Szczarba, The group $K_{3}(Z)$ is cyclic of order forty-eight, Ann. of Math. (2) 104 (1976), no. 1, 31-60. MR 0442934

[LS76b] _ On the homology and cohomology of congruence subgroups, Invent. Math. 33 (1976), no. 1, 15-53. MR 0422498

[LS78] _ On the torsion in $K_{4}(\mathbf{Z})$ and $K_{5}(\mathbf{Z})$, Duke Math. J. 45 (1978), no. 1, 101-129. MR 0491893

[MPP] Jeremy Miller, Peter Patzt, and Andrew Putman, On the top dimensional cohomology groups of congruence subgroups of $\mathrm{S} L_{n}(\mathbb{Z})$, preprint, https://arxiv.org/abs/1909.02661.

[MPW19] Jeremy Miller, Peter Patzt, and Jennifer C. H. Wilson, Central stability for the homology of congruence subgroups and the second homology of Torelli groups, Adv. Math. 354 (2019), 106740, 45. MR 3992366 
[MPWY] Jeremy Miller, Peter Patzt, Jennifer C. H. Wilson, and Dan Yasaki, Non-integrality of some steinberg modules, Preprint, to appear in J. Topol., https://arxiv.org/abs/1810.07683.

[Nag19] Rohit Nagpal, VI-modules in nondescribing characteristic, part I, Algebra Number Theory 13 (2019), no. 9, 2151-2189. MR 4039499

[Par97] Andrei Paraschivescu, On a generalization of the double coset formula, Duke Math. J. 89 (1997), no. 1, 1-8. MR 1458968

[Pat] Peter Patzt, Central stability homology, preprint, to appear in Math Z., https://arxiv.org/abs/1704.04128v2.

[Pri70] Stewart B. Priddy, Koszul resolutions, Trans. Amer. Math. Soc. 152 (1970), 39-60. MR 0265437

[PS] Andrew Putman and Daniel Studenmund, The dualizing module and top-dimensional cohomology group of $G L_{n}(\mathcal{O})$, preprint, https://arxiv.org/abs/1909.01217.

[PS17] Andrew Putman and Steven V. Sam, Representation stability and finite linear groups, Duke Math. J. 166 (2017), no. 13, 2521-2598. MR 3703435

[Put15] Andrew Putman, Stability in the homology of congruence subgroups, Invent. Math. 202 (2015), no. 3, 987-1027. MR 3425385

[Qui73] Daniel Quillen, Finite generation of the groups $K_{i}$ of rings of algebraic integers, Algebraic $K$-theory, I: Higher K-theories (Proc. Conf., Battelle Memorial Inst., Seattle, Wash., 1972), Springer, Berlin, 1973 , pp. 179-198. Lecture Notes in Math., Vol. 341. MR 0349812

[SEVKM] Mathieu Dutour Sikirić, Philippe Elbaz-Vincent, Alexander Kupers, and Jacques Martinet, Voronoi complexes in higher dimensions, cohomology of $G L_{N}(Z)$ for $N \geq 8$ and the triviality of $K_{8}(Z)$, preprint.

[SS15] Steven V. Sam and Andrew Snowden, Stability patterns in representation theory, Forum Math. Sigma 3 (2015), e11, 108. MR 3376738

150 N University Street West Lafayette, IN-47904 USA, Department of Mathematics, Purdue UniVERSITY, USA

E-mail address: jeremykmiller@purdue.edu

530 Church St, Ann Arbor, Mi 48109, Department of Mathematics, University of Michigan, USA

E-mail address: rohitna@umich.edu

150 N University Street West Lafayette, IN-47904 USA, Department of Mathematics, Purdue UniVERSITY, USA

E-mail address: ppatzt@purdue.edu 